\title{
Confronting smokers with previously undetected airflow limitation for smoking cessation
}

Citation for published version (APA):

Kotz, D. (2008). Confronting smokers with previously undetected airflow limitation for smoking cessation. [Doctoral Thesis, Maastricht University]. Datawyse / Universitaire Pers Maastricht. https://doi.org/10.26481/dis.20081121dk

Document status and date:

Published: 01/01/2008

DOI:

$10.26481 / \mathrm{dis} .20081121 \mathrm{dk}$

Document Version:

Publisher's PDF, also known as Version of record

\section{Please check the document version of this publication:}

- A submitted manuscript is the version of the article upon submission and before peer-review. There can be important differences between the submitted version and the official published version of record.

People interested in the research are advised to contact the author for the final version of the publication, or visit the DOI to the publisher's website.

- The final author version and the galley proof are versions of the publication after peer review.

- The final published version features the final layout of the paper including the volume, issue and page numbers.

Link to publication

\footnotetext{
General rights rights.

- You may freely distribute the URL identifying the publication in the public portal. please follow below link for the End User Agreement:

www.umlib.nl/taverne-license

Take down policy

If you believe that this document breaches copyright please contact us at:

repository@maastrichtuniversity.nl

providing details and we will investigate your claim.
}

Copyright and moral rights for the publications made accessible in the public portal are retained by the authors and/or other copyright owners and it is a condition of accessing publications that users recognise and abide by the legal requirements associated with these

- Users may download and print one copy of any publication from the public portal for the purpose of private study or research.

- You may not further distribute the material or use it for any profit-making activity or commercial gain

If the publication is distributed under the terms of Article $25 \mathrm{fa}$ of the Dutch Copyright Act, indicated by the "Taverne" license above, 
CONFRONTING SMOKERS

WITH PREVIOUSLY UNDETECTED

AIRFLOW LIMITATION

FOR SMOKING CESSATION 
The study of which results are presented in chapters 4 through 9 from this thesis was funded by the Netherlands Asthma Foundation (3.4.03.27), Partners in Care Solutions for COPD (PICASSO - an initiative of the School for Public Health and Primary Care (CAPHRI), Boehringer Ingelheim, and Pfizer), and Maastricht University Medical Centre. The study presented in chapter 3 was funded by Pfizer, the study in chapter 2 by the Dutch "Partnership Stop met Roken", and the study in chapter 10 by Cancer Research UK, Pfizer, J\&J, and GSK.

The work presented in this dissertation was conducted under auspices of the School for Public Health and Primary Care (CAPHRI), at Maastricht University Medical Centre, the Netherlands. CAPHRI is part of the Netherlands School of Primary Care Research (CaRe), which has been acknowledged since 1995 by the Royal Netherlands Academy of Arts and Sciences (KNAW).

The publication of this thesis was financially supported by the Netherlands Asthma Foundation (3.4.03.27), Pfizer and Boehringer Ingelheim.

ISBN: 9789052787817

Layout: Daniel Kotz

Cover: Bas Verhage

Printed by: Dataywse | Universitaire Pers Maastricht

Copyright (C) Daniel Kotz, 2008. All rights reserved. No part of this thesis may be reproduced or transmitted in any form or by any means, electronic or mechanical, including photocopying, recording, or any information storage or retrieval system, without permission in writing from the author, or, when appropriate, from the publishers of the publications. 


\section{CONFRONTING SMOKERS \\ WITH PREVIOUSLY UNDETECTED AIRFLOW LIMITATION FOR SMOKING CESSATION}

\section{Proefschrift}

Ter verkrijging van de graad van doctor aan de Universiteit Maastricht, op gezag van de Rector Magnificus, Prof. mr. G.P.M.F. Mols, volgens het besluit van het College van Decanen, in het openbaar te verdedigen op vrijdag, 21 november 2008 om 14.00 uur

door

\section{Daniel Kotz}

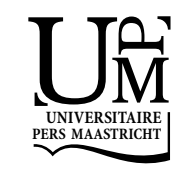




\section{Promotor}

Prof. dr. C.P. van Schayck

\section{Copromotores}

Dr. G. Wesseling

Dr. M.J.H. Huibers

\section{Beoordelingscommissie}

Prof. dr. J.A. Knottnerus, voorzitter

Dr. IJ. Kant

Prof. dr. D.S. Postma (University Medical Centre Groningen)

Prof. dr. H. de Vries

Prof. dr. R.J. West (University College London) 
FÜR MEINE ELTERN 



\section{TABLE OF CONTENTS}

Chapter 1 Introduction $\quad 9$

\section{Prologue}

$\begin{array}{lll}\text { Chapter } 2 \text { Smoking cessation practices of Dutch general practitioners, } & 19\end{array}$ cardiologists and lung physicians

Chapter 3 Smoking cessation treatment by Dutch respiratory nurses: 31 reported practice, attitudes and perceived effectiveness

\section{Main part}

Chapter 4 Principles of confrontational counselling in smokers with chronic obstructive pulmonary disease (COPD)

Chapter 5 Efficacy of confrontational counselling for smoking cessation in smokers with previously undiagnosed mild to moderate airflow limitation: study protocol of a randomized controlled trial

Chapter 6 External validation of a chronic obstructive pulmonary disease diagnostic questionnaire

Chapter 7 Confronting smokers with previously undetected COPD does not increase prolonged smoking cessation rates: a randomized controlled trial

Chapter 8 What mediates the effect of confrontational counselling on smoking cessation in smokers with COPD?

Chapter 9 Ethical analysis of the justifiability of labelling with COPD for smoking cessation

\section{Epilogue}

Chapter 10 Explaining the social gradient in smoking cessation: it's not in the trying, but in the succeeding

Chapter 11 General discussion

Summary

Samenvatting

Acknowledgements

About the author

List of publications 

Chapter 1

Introduction 


\section{BACKGROUND}

Cigarette smoking is the single most preventable cause of premature death in Western society. In the Netherlands, about 20.000 deaths each year (i.e. approximately $15 \%$ of all deaths) can be attributed to smoking-related diseases including lung cancer, cardiovascular disease, and chronic obstructive pulmonary disease (COPD). ${ }^{1}$

COPD is a preventable and treatable disease which is characterized by airflow limitation that is not fully reversible. ${ }^{2}$ It is the fifth leading cause of death worldwide ${ }^{3}$, and projections for 2020 indicate a further increase in global mortality, placing COPD on the third position of lethal diseases. ${ }^{4}$ Cigarette smoking is by far the most important risk factor for COPD; at least $15 \%$ to $50 \%$ of smokers are estimated to develop COPD during their lifetime. ${ }^{5}$ Smoking cessation is the single most effective way to reduce the risk of developing COPD and to affect the outcome in patients at all stages of the disease. ${ }^{6,7}$

Despite the well-known health risks, many continue to smoke. The rate of decline of smoking prevalence in the Netherlands has slowed down considerably since the 1990 s and has settled down at about $29 \%$ in recent years. ${ }^{8}$ Whereas most smokers want to quit, only a fraction of those who try remain long-term abstinent, mainly because of nicotine and tobacco dependence. Nicotine is the main component in cigarettes that contributes to addiction; it acts on nicotinic cholinergic receptors in the brain to release dopamine and other neurotransmitters that sustain addiction. ${ }^{9}$

The two main types of treatment that can help addicted smokers to quit are pharmacological and behavioural treatment. In the Netherlands, nicotine replacement therapy (e.g., nicotine gum or patch), bupropion, and nortriptyline are recommended evidence-based pharmacological treatments for smoking cessation. ${ }^{10}$ Among the behavioural treatments are a minimum intervention strategy for general practice (the so-called "H-MIS") and a more intensive intervention strategy for the pulmonary outpatients' clinic or nursing consultants (the so-called "L-MIS"). ${ }^{10}$ The L-MIS has been specifically designed for smokers with chronic lung diseases such as COPD. But even when treated with a combination of pharmacological and behavioural treatment, about $75 \%$ or more of smokers making an attempt to quit relapse within the first year. These relapse rates are supposed to be even higher in smokers with COPD because of their longer smoking history, higher levels of nicotine addiction, and higher prevalence of psycho-social co-morbidities such as depression; all these factors are known to be associated with relapse.

It is important to improve smoking cessation strategies, especially in smokers with COPD, who have a greater and more urgent need to stop smoking than the average smoker. ${ }^{11} \mathrm{~A}$ potentially successful motivational tool may be spirometry, the gold 
standard for the diagnosis and assessment of COPD. ${ }^{2}$ While most smokers acknowledge that smoking is dangerous, many trivialize their own perceived risk of the disease, deny, or avoid information about the dangers of smoking in order to reduce cognitive dissonance. ${ }^{12-16}$ One might therefore expect that confronting smokers with an objectively identified negative consequence of smoking by spirometry (airflow limitation, COPD) positively affects the outcome of their attempt to quit. This idea was already proposed in the 1960 s by Peters and Ferris who argued that assessing the negative effects of smoking on lung function "might serve as a lever to influence the young adult to reduce his smoking habits". ${ }^{17}$ Since then, various studies have been performed to study the efficacy of spirometry for smoking cessation. The results, however, are inconclusive. ${ }^{18}$ Also, previous studies have one or more important methodological limitations such as unstandardized counselling intensity, incomparable or uncontrolled use of pharmacological aids for smoking cessation between experimental and control group, and different (or unclear) baseline levels of lung function and motivation to quit smoking.

We conducted a randomized controlled trial to test the efficacy of confronting smokers with spirometry for smoking cessation (trial register: Netherlands Trial Register ISRCTN 64481813). The trial was designed to account for potential methodological biases and to isolate the effects of labelling with disease.

\section{OUTLINE OF THE THESIS}

The randomized controlled trial on the efficacy of confrontational counselling is the main part of this $\mathrm{PhD}$ thesis. The research that preceded this work is depicted in the prologue - observational research on the smoking cessation practices of Dutch health care providers. In the epilogue we look beyond the specific approach of confrontational counselling for smoking cessation and focus on a more general phenomenon and important societal problem, using data from a UK national survey: the social inequalities of smoking prevalence and smoking cessation.

\section{Prologue}

According to national and international guidelines on the treatment of tobacco dependence, health care providers can play an important role in reducing the prevalence of smoking. ${ }^{10,19,20} \mathrm{~A}$ large proportion of the population has regular contact with health care providers who could well use this contact as an opportunity to encourage patients to stop smoking. This is particularly the case in patients with smokingrelated diseases such as COPD. Health care providers who see such patients are GPs, lung physicians, cardiologists, and respiratory nurses. However, it is unclear what their smoking cessation practices, attitudes, and perceived effectiveness are. To answer these questions, we conducted national surveys among 834 GPs, 300 cardi- 
ologists, 258 lung physicians; the results from these three surveys are combined in chapter 2. Results from a national survey among 254 respiratory nurses are presented in chapter 3.

\section{Main part}

Should health care providers, especially in primary care, use spirometry for early detection of COPD and as a motivational tool for smoking cessation? Our trial was meant to answer this question. We hypothesised that early detection of COPD and confrontation with spirometry for smoking cessation may be effective if certain conditions, described in chapter 4, are met. Confronting patients with COPD should not be an isolated approach but should be integrated into state-of-the-art smoking cessation treatment consisting of individual counselling in combination with pharmacotherapy for smoking cessation. We termed this approach "confrontational counselling", and tested its efficacy in a randomized controlled trial (see chapter $\mathbf{5}$ for a detailed protocol).

All smokers from our trial had previously undetected airflow limitation. It has been suggested that early detection of smokers with COPD may be enhanced by using questionnaires to identify patients with a high likelihood of having airflow limitation before using spirometry. ${ }^{21,22}$ GPs may use a questionnaire that is recommended by the International Primary Care Airways Group. ${ }^{23-25}$ Before this new diagnostic questionnaire can be accepted and applied reliably in clinical practice, the analysis of the underlying model must be repeated on new data collected from an appropriate sample of subjects from a different setting. We performed this external validation in the smokers we screened for eligibility for taking part in the trial (chapter 6).

We screened more than 1,700 smokers, 296 of whom finally participated. The trial was designed to assess the "net" effect of confronting smokers with spirometry by comparing medium intensity confrontational counselling delivered by a respiratory nurse combined with nortriptyline for smoking cessation (experimental group) with medium intensity health education and promotion delivered by a respiratory nurse combined with nortriptyline for smoking cessation (control group 1). The effects of both medium intensity treatments delivered by a respiratory nurse were compared with low intensity "care as usual" for smoking cessation by the general practitioner (control group 2). Only participants from the experimental group were confronted with their abnormal lung function. The primary research question was whether confrontational counselling increased prolonged abstinence rates from smoking during a period of 12 months after the target quit date (chapter 7).

We were not only interested in finding out whether or not confrontational counselling was effective but also how it might work. When we designed the intervention, we hypothesised that confrontational counselling - through labelling with a potentially 
life-threatening illness status - increases risk perception and health concerns and decreases self-exempting beliefs in smokers, changes which in turn may account for abstinence from smoking. To analyse these mechanisms of change, we performed a mediation analysis on the effect of confrontational counselling on short-term abstinence from smoking, five weeks after the target quit date (chapter 8).

Irrespectively of its efficacy, it is worth considering whether an approach of early detection and labelling with COPD is justifiable. Labelling with disease - confronting healthy subjects with a previously undetected or unrecognized disease status - has long been an issue of debate in the medical field. In most cases, the debate has focused on the potential adverse effects of labelling. With regard to early detection of COPD by means of spirometry, it has been argued that widespread spirometry is likely to label a large number of individuals with disease, resulting in considerable testing, treatment costs, and health-care resource utilization. ${ }^{18,26,27}$ However, it is also possible that the act of labelling can be used as a motivational tool to change unhealthy behaviour, in our case to motivate smokers to quit smoking. Since empirical data of patients' perspectives in this debate are rare, we conducted semistructured ethical exit interviews on this subject in 205 of our trial participants. The objective was to assess the opinions of these participants on the effectiveness of spirometry for smoking cessation, the justification of early detection of airflow limitation in smokers, and the impact of confrontation with COPD (chapter 9).

\section{Epilogue}

As stated before, a considerable proportion of the population smokes despite the well-known health risks. Furthermore, there is a large socio-economic gradient in smoking in the Netherlands as in other developed countries with the highest prevalence of smoking in half-skilled and unskilled manual labour (44\% in the Netherlands in 2007) and the lowest prevalence in highly-skilled white-collar workers $(27 \%){ }^{8}$ This gradient is estimated to be responsible for half of socio-economic differences in mortality in men aged 35-69 years in developed countries ${ }^{28}$. An important future goal is to reduce social health inequalities that are caused by smoking. Reducing inequalities in smoking prevalence requires a better understanding of what is causing it. Are smokers in more deprived socio-economic groups less motivated to stop? Do they make less use of smoking cessation therapies? We used data from 6,950 respondents to a UK cross-sectional household survey to examine the variation across social grades in: rates of attempts to stop smoking; use of pharmacological and behavioural treatment for smoking cessation; and success rates of quit attempts (chapter 10). 


\section{REFERENCES}

1 Leent-Loenen HMJA. Roken samengevat. In: Volksgezondheid Toekomst Verkenning. Nationaal Kompas Volksgezondheid. Bilthoven: RIVM, 2007

2 Global Initiative for Chronic Obstructive Lung Disease. Global strategy for the diagnosis, management, and prevention of chronic pulmonary disease. Executive summary (http://www.goldcopd.com): NHLBI / WHO, 2006

3 World Health Organisation (WHO). The World Health Report. Reducing risks, promoting healthy life. Geneva: WHO, 2002

4 Murray CJ, Lopez AD. Alternative projections of mortality and disability by cause 1990-2020: Global Burden of Disease Study. Lancet 1997; 349:1498-1504

5 Rennard SI, Vestbo J. COPD: the dangerous underestimate of 15\%. Lancet 2006; 367:1216-1219

6 Pauwels RA, Buist AS, Calverley PM, et al. Global strategy for the diagnosis, management, and prevention of chronic obstructive pulmonary disease. NHLBI/WHO Global Initiative for Chronic Obstructive Lung Disease (GOLD) Workshop summary. Am J Respir Crit Care Med 2001; 163:1256-1276

7 Anthonisen NR, Connett JE, Kiley JP, et al. Effects of smoking intervention and the use of an inhaled anticholinergic bronchodilator on the rate of decline of FEV1. The Lung Health Study. J Am Med Assoc 1994; 272:1497-1505

8 Centraal Bureau voor de Statistiek [Statistics Netherlands] (CBS). Gerapporteerde gezondheid en leefstijl 2008. [Reported health and life style 2008.]

9 Benowitz NL. Clinical pharmacology of nicotine: implications for understanding, preventing, and treating tobacco addiction. Clin Pharmacol Ther 2008; 83:531-541

10 Kwaliteitsinstituut voor de Gezondheidszorg CBO. Richtlijn Behandeling van Tabaksverslaving. [Guideline Treating Tobacco Dependence.]. Utrecht: Kwaliteitsinstituut voor de Gezondheidszorg CBO, 2004

11 Tonnesen P, Carrozzi L, Fagerstrom KO, et al. Smoking cessation in patients with respiratory diseases: a high priority, integral component of therapy. ERS task force guideline. Eur Respir J 2007; 29:390-417

12 Halpern MT. Effect of smoking characteristics on cognitive dissonance in current and former smokers. Addict Behav 1994; 19:209-217

13 McMaster C, Lee C. Cognitive dissonance in tobacco smokers. Addict Behav 1991; 16:349-353

14 Chapman S, Wong WL, Smith W. Self-exempting beliefs about smoking and health: differences between smokers and ex-smokers. Am J Public Health 1993; 83

15 Lee C. Perceptions of immunity to disease in adult smokers. J Behav Med 1989; 12:267-277

16 McCoy SB, Gibbons FX, Reis TJ, et al. Perceptions of smoking risk as a function of smoking status. J Behav Med 1992; 15:469-488

17 Peters JM, Ferris BG, Jr. Smoking, pulmonary function, and respiratory symptoms in a college-age group. Am Rev Respir Dis 1967; 95:774-782

18 Wilt TJ, Niewoehner D, Kane RL, et al. Spirometry as a motivational tool to improve smoking cessation rates: a systematic review of the literature. Nicotine Tob Res 2007; 9:21-32

19 Fiore MC, Bailey WC, Cohen SJ, et al. Treating tobacco use and dependence. Clinical Practical Guideline. Rockville, MD: US Department of Health and Human Services, 2000

20 Chavannes NH, Kaper J, Frijling BD, et al. NHG-Standaard Stoppen met roken. Huisarts en Wetenschap 2007; 50:306-314

21 van Schayck CP, Loozen JM, Wagena E, et al. Detecting patients at a high risk of developing chronic obstructive pulmonary disease in general practice: cross sectional case finding study. BMJ 2002; 324:13701374

22 van Schayck CP, Halbert RJ, Nordyke RJ, et al. Comparison of existing symptom-based questionnaires for identifying COPD in the general practice setting. Respirology 2005; 10:323-333

23 Price DB, Tinkelman DG, Halbert RJ, et al. Symptom-based questionnaire for identifying chronic obstructive pulmonary disease in smokers. Respiration 2006; 73:285-295

24 Price DB, Tinkelman DG, Nordyke RJ, et al. Scoring System and Clinical Application of COPD Diagnostic Questionnaires. Chest 2006; 129:1531-1539

25 International Primary Care Airways Group (IPAG). Chronic airways diseases. A guide for primary care physicians., 2005 
26 Wilt TJ, Niewoehner D, Kim C-B, et al. Use of Spirometry for case finding, diagnosis, and management of chronic obstructive pulmonary disease (COPD). Evidence Report/Technology Assessment No. 121 (Prepared by the Minnesota Evidence-based Practice Center under Contract No. 290-02-0009.) AHRQ Publication No. 05-E017-2. Rockville, MD.: Agency for Healthcare Research and Quality, 2005

27 Enright P. Does screening for COPD by primary care physicians have the potential to cause more harm than good? Chest 2006; 129:833-835

28 Jha $\mathrm{P}$, Peto $\mathrm{R}$, Zatonski $\mathrm{W}$, et al. Social inequalities in male mortality, and in male mortality from smoking: indirect estimation from national death rates in England and Wales, Poland, and North America. Lancet 2006; 368:367-370 

PROLOGUE 



\section{Chapter 2}

\section{Smoking cessation practices of Dutch general practitioners, cardiologists and lung physicians}

Daniel Kotz ${ }^{1}$

Edwin J. Wagena ${ }^{2}$

Geertjan Wesseling ${ }^{3}$

Published in: Respiratory Medicine 2007; 101 (3): 568-573

${ }^{1}$ Department of General Practice, School for Public Health and Primary Care (CAPHRI), Maastricht University Medical Centre, Maastricht, the Netherlands. ${ }^{2}$ Pulmonary Rehabilitation Centre Hornerheide, Horn, the Netherlands (since January 2006, E.J. Wagena has been affiliated with Astellas Pharma Europe B.V., Leiderdorp, the Netherlands). ${ }^{3}$ Department of Respiratory Medicine, Maastricht University Medical Centre, Maastricht, the Netherlands 


\section{ABSTRACT}

\section{Study objectives}

To assess and compare the smoking cessation practices and smoking behaviour of Dutch GPs, cardiologists, and lung physicians.

\section{Methods}

We conducted questionnaire surveys among a random sample of 2000 Dutch GPs, all Dutch cardiologists $(\mathrm{N}=594)$, and all Dutch lung physicians $(\mathrm{N}=375)$.

\section{Results}

In total, 834 GPs (41.7\%), 300 cardiologists (50.5\%), and 258 lung physicians $(68.8 \%)$ filled out and returned the questionnaire. The prevalence of current smokers was $8.2 \%$ among GPs, $4.3 \%$ among cardiologists, and 3.5\% among lung physicians. Of the pharmacological aids for smoking cessation, physicians recommended bupropion most frequently, followed by nicotine patches and nicotine gum. More lung physicians recommended the use of these three aids $(67.0 \%, 36.3 \%$ and $18.2 \%$ respectively) than GPs $(65.7 \%, 18.7 \%$ and $9.8 \%$ respectively), and than cardiologists (31.6\%, $19.7 \%$ and $13.2 \%$ respectively). A higher proportion of lung physicians $(69.3 \%)$ had referred at least one smoker to a nurse for smoking cessation treatment than cardiologists (25\%), and than GPs (11.3\%).

\section{Conclusions}

Based on this national survey, one may conclude that the prevalence of current smoking among Dutch physicians is relatively low and has further decreased since 1988. Dutch GPs, cardiologists and lung physicians mainly use interventions for smoking cessation that are easy to administer and are not very time consuming. Furthermore, more lung physicians than GPs and cardiologists recommend the use of bupropion, nicotine patch, and nicotine gum. When designing interventions for smoking cessation, one should take into account that physicians are often reluctant to provide interventions which demand much time. Therefore, intensive counselling of smokers who want to quit smoking may be more feasible for trained nonphysicians, such as nurses. 


\section{INTRODUCTION}

International smoking cessation guidelines recommend that health professionals should asses the smoking status of their patients and advise smokers to stop. ${ }^{1-3}$ They should also facilitate a quit attempt by recommending the use of pharmacotherapy (bupropion, nicotine replacement therapy [NRT]), offering brief counselling, and arranging follow up visits when appropriate. According to the recently developed Dutch guideline on the treatment of tobacco dependence ${ }^{4}$, general practitioners (GPs) can play an important role in reducing smoking prevalence because about $75 \%$ of the population consult their GP at least once a year. ${ }^{5}$ Specialists, such as lung physicians and cardiologists, could well use hospitalization as an opportunity to encourage their patients to stop smoking. ${ }^{3,6}$ Not only because the illness or condition resulting in hospitalization may have been caused or complicated by smoking, highlighting the patient's personal vulnerability to the health risks of smoking, but also because hospitalized smokers are temporarily housed in a smoke-free environment, offering unique opportunities for cessation treatment.

In the Netherlands, few data are available about the practice of GPs, cardiologists and lung physicians regarding the treatment of tobacco dependence. We could only find one study, conducted almost 20 years ago, describing the smoking cessation practice of Dutch GPs. ${ }^{7}$ Since then, new smoking cessation methods have been developed and several reviews into their effectiveness have been published. ${ }^{8-12}$

We conducted three questionnaire surveys among a random sample of Dutch GPs, all Dutch cardiologists and all Dutch lung physicians to assess and compare the smoking cessation practices and smoking behaviour of these health professionals.

\section{METHODS}

\section{Questionnaire administration}

From May 2002 through January 2003, we conducted questionnaire surveys including a random sample of 2000 GPs (approximately $27 \%$ of all registered GPs in the Netherlands), all 594 Dutch cardiologists, and all 375 Dutch lung physicians. Electronic files with the names and addresses of registered GPs, cardiologists, and lung physicians were provided by the Netherlands Institute of Health Services Research (NIVEL), the Dutch Society of Cardiology (NVVC), and the Dutch Thoracic Society (NVALT), respectively. We used a personalized introduction letter explaining the objectives of the study and self-addressed envelopes. Regarding the surveys among cardiologists and lung physicians, we repeatedly sent mailed reminders with an abridged version of the questionnaire to all non-responders in order to increase the response rate. Regarding the survey among GPs, we did not use mailed reminders. 
Instead, we sent a questionnaire containing only questions on smoking behaviour to a random sample of $100 \mathrm{GPs}$ from the non-response group. A gift voucher of EUR 5,(USD 6,52) was sent along.

\section{Questionnaire development and content}

The questionnaire consisted of three parts and was pre-tested by epidemiologists and physicians. Its completion time turned out to be 5 to 10 minutes. The first part contained questions about the physicians' demographic and work-related characteristics. In the second part, we asked which of the existing pharmacotherapeutical and behavioural interventions for smoking cessation they had used and how many smokers they had advised to stop smoking. In order to minimize recall bias, these questions referred to the last four weeks preceding the questionnaire. In the third part, we asked the physicians about their own smoking status. We defined "current smokers" as those physicians who reported to smoke cigarettes every day or occasionally. "Past smokers" were those non-smokers who used to smoke cigarettes every day or occasionally in the past. "Never smokers" were those non-smokers who had never smoked cigarettes.

\section{Data analysis}

We used Mann-Whitney U-tests to compare the mean number of smokers that were advised to stop smoking by GPs, cardiologists, and lung physicians. Overall Chisquared tests were performed to compare the frequency of reporting the use of pharmacotherapeutical and behavioural interventions for smoking cessation between the three study groups. In case of a statistically significant overall test $(p<0.05)$, pair-wise Chi-squared tests were performed in order to determine which of the study groups differed from one another. Likewise, Chi-squared tests were performed to compare the smoking status of the study groups. In case less than $80 \%$ of cells had expected values of at least 5, Fisher's exact test was used to determine P-values. Cases were excluded analysis-by-analysis (e.g., a case was excluded only for the analysis in which it had a missing value). All analyses were performed using the Statistical Package for Social Sciences (SPSS) 11.0.

\section{RESULTS}

In total, 758 GPs (37.9\%) filled out and returned the questionnaire. 76 GPs (76\%) from the non-response group filled out and returned the short questionnaire containing only questions on smoking behaviour. From the 594 Dutch cardiologists, 300 (50.5\%) filled out and returned a questionnaire. Thirty-five cardiologists returned the long version of the questionnaire containing questions about pharmacological and behavioural interventions as well as smoking behaviour. Forty-one cardiologists returned the abridged version of the questionnaire containing questions about 
pharmacological and behavioural interventions only. A total of 129 cardiologists returned the abridged version of the questionnaire containing questions about smoking behaviour only. Of the 375 lung physicians, 258 (68.8\%) filled out and returned a questionnaire. Two hundred and sixteen lung physicians returned the abridged version of the questionnaire containing questions about pharmacological and behavioural interventions only.

\section{Demographic and work-related characteristics}

Table 1 shows the demographic and work-related characteristics of the three study groups. $69.3 \%(n=525)$ of the GPs were male, compared to $88.3 \%(n=264)$ of the cardiologists and $82.6 \%(n=213)$ of the lung physicians. The mean ages in the three samples were $45.9(\mathrm{SD}=7.3), 48.3(\mathrm{SD}=7.1)$, and 47.0 years $(\mathrm{SD}=7.0)$, respectively.

\section{Number of smokers advised to stop smoking}

On average, GPs had advised 9.2 patients $(S D=9.4)$ to stop smoking during the four weeks preceding the questionnaire. Cardiologists had advised 24.3 patients to do so $(S D=30.0)$, a significant difference compared to GPs $(z=-12.74, p<0.001)$. Lung physicians had advised 38.8 patients $(S D=40.9)$, significantly more than cardiologists $(z=-4.26, p<0.001)$ and GPs $(z=-13.83, p<0.001)$.

\section{Recommendation or prescription of pharmacotherapy for smoking cessation}

Table 2 shows the number and percentage of physicians who had prescribed or recommended pharmacotherapy for smoking cessation at least once during the four weeks preceding the questionnaire. The use of bupropion had been recommended most frequently, followed by nicotine patches and nicotine gum. The use of bupropion had been recommended by a significantly higher proportion of lung physicians $\left(n=124,67.0 \%, \chi^{2}=27.57, d f=1, p<0.001\right)$ and GPs $\left(n=495,65.7 \%, \chi^{2}=34.41\right.$, $\mathrm{df}=1, \mathrm{p}<0.001)$ than cardiologists $(n=24,31.6 \%)$. The use of nicotine patches had been recommended by a significantly higher proportion of lung physicians $(n=66$, $36.3 \%)$ than GPs $\left(n=139,18.7 \%, \chi^{2}=26.12, d f=1, p<0.001\right)$, and than cardiologists $\left(n=15,19.7 \%, \chi^{2}=6.80, d f=1, p<0.01\right)$. The use of nicotine gum had been recommended by a significantly higher proportion of lung physicians $(n=33,18.2 \%)$ than GPs $\left(n=72,9.8 \%, \chi^{2}=10.18, d f=1, p<0.01\right)$. Only a few GPs and lung physicians, and no cardiologists had prescribed or recommended nortriptyline, nicotine tablet, microtab (sublingual tablet), or nicotine inhaler.

We further analyzed differences in demographic and work-related characteristics with regard to the prescription of pharmacotherapy. We limited the analysis to the prescription of bupropion because it had been recommended most frequently. This stratification was only suitable for the GP data which included a large number of respondents. There were no significant differences in the prescription of bupropion 
concerning sex, age and smoking status. However, significantly more GPs working in a solo-practice $(n=198,73.1 \%$ ) had prescribed bupropion than GPs working in a duo-practice $(n=160,59.5 \%)$ and than GPs working in a group-practice $(n=134$, 64.7, $\left.\chi^{2}=11.24, d f=2, p<0.01\right)$.

\section{Use of behavioural interventions for smoking cessation}

Table 3 shows the numbers and percentages of physicians who had used behavioural interventions for smoking cessation at least once during the four weeks preceding the questionnaire. 366 GPs (48.6\%), 38 cardiologists $(50.0 \%)$, and 85 lung physicians (42.3\%) had provided smokers with self-help materials. 243 GPs (32.4\%), 19 cardiologists (25.0\%), and 53 lung physicians (26.9\%) had used the Minimal Intervention Strategy (MIS), which is a minimum contact intervention that health care providers can use to help smokers quit. Significantly more lung physicians $(n=142$, $69.3 \%$ ) had referred at least one smoker to a nurse for smoking cessation treatment than cardiologists $\left(n=19,25 \%, \chi^{2}=44.41, d f=1, p<0.001\right)$, and than GPs $(n=85$, $\left.11.3 \%, \chi^{2}=298.2, \mathrm{df}=1, \mathrm{p}<0.001\right)$. Significantly more cardiologists had referred smokers to a nurse than GPs $\left(\chi^{2}=11.71, \mathrm{df}=1, \mathrm{p}<0.001\right)$. We asked cardiologists and lung physicians if they had referred any smokers to their GPs for smoking cessation treatment. $36.8 \%(n=75)$ of the lung physicians and $42.1 \%(n=32)$ of the cardiologists gave an affirmative answer.

\section{Physicians' smoking status}

Table 4 shows the smoking status of Dutch GPs, lung physicians and cardiologists. The smoking prevalence was highest among GPs $(8.2 \%, n=62)$, and lowest among lung physicians $(3.5 \%, n=9)$. However, the prevalence of smokers among Dutch GPs is assumed to be higher, since the non-response analysis showed that 12 of the 76 (16\%) GPs reported that they currently smoked cigarettes. Only the difference between GPs and lung physicians in the distribution of current, past, and never smokers was statistically significant $\left(\chi^{2}=7.21, d f=2, p=0.03\right)$.

\section{DISCUSSION}

The results from the questionnaire surveys we conducted among Dutch GPs, lung physicians, and cardiologists showed that of the pharmacological aids for smoking cessation, physicians recommended bupropion most frequently. In comparison with cardiologists, almost twice as many GPs and lung physicians recommended the use of this pharmacological aid. Among the nicotine replacement therapies, the patch and the gum were most popular, although they were much less frequently recommended by Dutch physicians than bupropion. Results also indicated that behavioural interventions for smoking cessation were not very often used. The most frequently used behavioural interventions were those which are not very time-consuming: 
providing self-help materials and referring smokers to a nurse for further treatment. The prevalence of current smokers among Dutch physicians was relatively low. More GPs than lung physicians were current smokers.

To our knowledge, this national study is the first to compare the use of interventions for smoking cessation by GPs, cardiologists, and lung physicians. We sent questionnaires to all Dutch lung physicians, all Dutch cardiologists, and to a random sample of approximately $27 \%$ of all Dutch GPs. Of the existing pharmacological aids for smoking cessation, bupropion was by far the most popular among Dutch physicians. One possible explanation might be that bupropion is easy to use and does not demand much time from the physician. This explanation is supported by our finding that the behavioural interventions most commonly used by physicians were self-help materials, which are also easy to use and are not very time-consuming. Bupropion was registered as a therapy for smoking cessation in the Netherlands in 1999. The marketing campaigns of the pharmaceutical company might also explain that bupropion has been widely prescribed by physicians, although the drug had some negative publicity in the Netherlands in 2001 due to suspected adverse reactions. ${ }^{13,14}$ Nortriptyline, a generic non-patent medicine, was prescribed by very few physicians, though it has been shown to be an efficacious and cheap alternative to bupropion. ${ }^{8,15}$ Sixty-nine percent of Dutch lung physicians and $25 \%$ of Dutch cardiologists referred smokers to a pulmonary nurse for assistance during a quit attempt. Assuming a dose-response relation between the number and duration of counselling sessions for tobacco dependence and their efficacy ${ }^{16}$, pulmonary nurses can play an important role in helping smokers to quit. One of the reasons is that they can probably spend more time with a smoker than the physician can. ${ }^{17}$

In the year 2004, shortly after our surveys, the Dutch guideline for the treatment of tobacco dependence was published. ${ }^{18}$ The national organizations of GPs (NHG), lung physicians (NVALT), and cardiologists (NVVC) had participated in the development. To what extent does the guideline reflect the reported practice of the respondents to our surveys? According to the guideline, health care providers should at least give smoking cessation advice to every new smoking patient and should repeat this annually. If one compares the number of smokers advised to stop smoking with the number of (smoking) patients these physicians are likely to see every month, one may believe that they do not give smoking cessation advice in every smoking patient. The guideline states that both bupropion and nortriptyline are effective and safe. However, due to their side effects, both drugs should not be prescribed as an aid for smoking cessation in the first place. Furthermore, prescription should be combined with at least two consultations with the physician. It remains doubtful, whether Dutch physicians (can) do so. The guideline acknowledges that health care providers have limited time for smoking cessation treatment. Therefore, the guideline recom- 
mends that nurse practitioners should be trained to become specialists for (intensive) smoking cessation interventions.

Between 1956 and 1988, the prevalence of smoking among Dutch GPs has decreased from $82 \%$ to $29 \% .{ }^{19}$ A similar trend has been found in Scandinavian countries. ${ }^{20}$ Although data on the prevalence of smoking among Dutch GPs between 1988 and 2002 are lacking, our results seem to indicate that the percentage of smoking GPs has decreased further. The prevalence of current smokers was higher among GPs than among lung physicians. One explanation might be that lung physicians are more regularly confronted with smoking-related diseases during daily practice. In the Netherlands, the prevalence of smoking among physicians (about 4 to $8 \%$ ) is much lower than among the general population (34\%). ${ }^{5}$ One possible explanation for this might be that physicians see themselves as role models for their patients by being non-smokers and by creating a smoke-free work environment. ${ }^{21,22}$ The prevalence of current smokers among Dutch physicians seems to be lower than in Germany $(18 \%)$ and Japan (20\%), but still higher than in the USA (3\%) and Finland (5\%). ${ }^{23-25}$

This study was limited by the low to moderate response rates of $38 \%$ to $69 \%$. Although such response rates are not unusual for studies in this field, one must be aware that selection bias may have occurred. The demographic and work-related characteristics of the three groups of respondents who participated in this study were similar to those of the total group of Dutch GPs, lung physicians and cardiologists. Moreover, we did not find differences in the mean number of patients advised to stop smoking by cardiologists and lung physicians who returned the first questionnaire compared to those who returned the reminder questionnaire. Nevertheless, if selection bias has occurred, it is likely that the responders represent a group of physicians that is more active in smoking cessation treatment than the nonresponders. In that case, the frequency of advice to quit smoking and of the prescription of smoking cessation aids we found may be an overestimation of the general situation. Our data may also present an underestimation of the prevalence of current and past smoking physicians. We found that the prevalence of smoking GPs among the random sample of 76 GPs from the non-response group was higher $(16 \%)$ than the prevalence among the total group of GPs who returned the questionnaire. We found a similar trend between early and late responders in the groups of cardiologists and lung physicians. We therefore expect the true prevalence of smoking physicians to be slightly higher than the prevalence we found. Data from cross-sectional studies with low response rates should always be interpreted with care. However, in the absence of studies with higher response rates, we think that our data present valuable information about the comparison of GPs, cardiologists, and lung physicians with regard to the use of interventions for smoking cessation. 
Based on this national survey, one may conclude that the prevalence of current smoking among Dutch physicians is relatively low and has further decreased since 1988. Dutch GPs, cardiologists and lung physicians mainly use interventions for smoking cessation that are easy to administer and are not very time consuming. Furthermore, more lung physicians than GPs and cardiologists recommend the use of bupropion, nicotine patch, and nicotine gum. When designing interventions for smoking cessation, one should take into account that physicians are often reluctant to provide interventions which demand much time. Therefore, intensive counselling of smokers who want to quit smoking may be more feasible for trained nonphysicians, such as nurses. 
Table 1: Demographic and work-related characteristics of the three study groups.

\begin{tabular}{lccc} 
Characteristics & $\begin{array}{c}\text { GPs } \\
(\mathrm{n}=758)\end{array}$ & $\begin{array}{c}\text { Cardiologists } \\
(\mathrm{n}=300)\end{array}$ & $\begin{array}{c}\text { Lung physicians } \\
(\mathrm{n}=258)\end{array}$ \\
Sex male, n (valid percentage) & $525(69.3)$ & $264(88.3)$ & $213(82.6)$ \\
Age, mean (SD) & $45.9(7.3)$ & $48.3(7.1)$ & $47.0(7.0)$ \\
Setting, n (valid percentage) & & & $\mathrm{n} / \mathrm{a}$ \\
Solo practice & $273(36.4)$ & $\mathrm{n} / \mathrm{a}$ & $\mathrm{n} / \mathrm{a}$ \\
Duo practice & $270(36.0)$ & $\mathrm{n} / \mathrm{a}$ & $\mathrm{n} / \mathrm{a}$ \\
Group practice & $208(27.7)$ & $\mathrm{n} / \mathrm{a}$ & $47(18.2)$ \\
University hospital & $\mathrm{n} / \mathrm{a}$ & $53(17.7)$ & $190(73.6)$ \\
District hospital & $\mathrm{n} / \mathrm{a}$ & $231(77.5)$ & $21(8.2)$ \\
Other & $\mathrm{n} / \mathrm{a}$ & $14(4.7)$ & \\
\hline
\end{tabular}

$\mathrm{n} / \mathrm{a}=$ not applicable

Table 2: Number and valid percentage of physicians who had prescribed or recommended pharmacotherapy for smoking cessation at least once during the preceding four weeks.

\begin{tabular}{lcccc} 
Pharmacotherapy & $\begin{array}{c}\text { GPs } \\
(n=758)\end{array}$ & $\begin{array}{c}\text { Cardiologists } \\
(n=76)\end{array}$ & $\begin{array}{c}\text { Lung physicians } \\
(\mathrm{n}=216)\end{array}$ & $\begin{array}{c}\text { Overall Chi-squared test }^{\dagger} \\
\text { and P-value }\end{array}$ \\
Bupropion & $495(65.7)$ & $24(31.6)$ & $124(67.0)$ & $\chi^{2}=35.99, \mathrm{df}=2, \mathrm{p}<0.001$ \\
Nicotine patch & $139(18.7)$ & $15(19.7)$ & $66(36.3)$ & $\chi^{2}=26.52, \mathrm{df}=2, \mathrm{p}<0.001$ \\
Nicotine gum & $72(9.8)$ & $10(13.3)$ & $33(18.2)$ & $\chi^{2}=10.31, \mathrm{df}=2, \mathrm{p}<0.01$ \\
\hline
\end{tabular}

${ }^{\dagger}$ The overall test compares the proportion of yes/no-responders across the three study groups.

Table 3: Number and valid percentage of physicians who had used behavioural interventions for smoking cessation at least once during the preceding four weeks.

\begin{tabular}{lcccc} 
Behavioural intervention & $\begin{array}{c}\text { GPs } \\
(\mathrm{n}=758)\end{array}$ & $\begin{array}{c}\text { Cardiologists } \\
(\mathrm{n}=76)\end{array}$ & $\begin{array}{c}\text { Lung physicians } \\
(\mathrm{n}=216)\end{array}$ & $\begin{array}{c}\text { Overall Chi-squared test } \\
\text { and } \mathrm{P} \text {-value }\end{array}$ \\
$\begin{array}{l}\text { Providing smokers with self- } \\
\text { help material }\end{array}$ & $366(48.6)$ & $38(50.0)$ & $85(42.3)$ & $\chi^{2}=2.75, \mathrm{df}=2, \mathrm{p}=0.25$ \\
$\begin{array}{l}\text { Using Minimal Intervention } \\
\begin{array}{l}\text { Strategy (MIS) } \\
\text { Referring smokers to practice } \\
\text { nurse / pulmonary nurse }\end{array}\end{array}$ & $243(32.4)$ & $19(25.0)$ & $53(26.9)$ & $\chi^{2}=3.50, \mathrm{df}=2, \mathrm{p}=0.17$ \\
$\begin{array}{l}\text { Referring smokers to telephone } \\
\text { counselling hotline }\end{array}$ & $76(11.3)$ & $19(25.0)$ & $142(69.3)$ & $\chi^{2}=297.5, \mathrm{df}=2, \mathrm{p}<0.001$ \\
\hline
\end{tabular}

${ }^{\top}$ The overall test compares the proportion of yes/no-responders across the three study groups.

Table 4: Number and valid percentage of current, past and never smokers among GPs, cardiologists and lung physicians.

\begin{tabular}{lccc} 
Smoking status $^{\dagger}$ & $\begin{array}{c}\text { General practitioners } \\
(n=758)\end{array}$ & $\begin{array}{c}\text { Cardiologists } \\
(n=164)\end{array}$ & $\begin{array}{c}\text { Lung physicians } \\
(n=258)\end{array}$ \\
Current smoker & $62(8.2)$ & $7(4.3)$ & $9(3.5)$ \\
Past smoker & $224(29.7)$ & $40(24.8)$ & $73(28.5)$ \\
Never smoker & $467(62.0)$ & $114(70.8)$ & $174(68.0)$ \\
\hline
\end{tabular}

${ }^{\dagger}$ Smoking refers only to the use of cigarettes. 


\section{REFERENCES}

1 West R, McNeill A, Raw M. Smoking cessation guidelines for health professionals: an update. Thorax 2000; 55:987-999

2 Raw M, McNeill A, West R. Smoking cessation: evidence based recommendations for the healthcare system. BMJ 1999; 318:182-185

3 U.S. Department of Health and Human Services. Clinical practice guideline. Treating tobacco use and dependence, 2000

4 Kwaliteitsinstituut voor de Gezondheidszorg CBO. Richtlijn Behandeling van Tabaksverslaving. [Guideline Treating Tobacco Dependence]. Utrecht: Kwaliteitsinstituut voor de Gezondheidszorg CBO, 2004

5 Centraal Bureau voor de Statistiek. Vademecum gezondheidsstatistiek Nederland 2003. [Basic guide to health statistics in the Netherlands 2003]. Voorburg/Heerlen: Centraal Bureau voor de Statistiek, 2004

6 Orleans CT, Kristeller JL, Gritz ER. Helping hospitalized smokers quit: new directions for treatment and research. J Consult Clin Psychol 1993; 61:778-789

7 Dekker HM, Dill AMS, Looman CWM, et al. Rookgedrag van huisartsen, specialisten en studenten in Rotterdam, 1989. [Smoking behaviour of family physicians, specialists and students in Rotterdam, 1989]. Ned Tijdschr Geneeskd 1990; 134:1495-1498

8 Hughes JR, Stead LF, Lancaster T. Antidepressants for smoking cessation (Cochrane Review). In: The Cochrane Library, Issue 3. Cichester, UK: John Wiley \& Sons Ltd. 2004

9 Lancaster T, Stead LF. Individual behavioural counselling for smoking cessation (Cochrane Review). In: The Cochrane Library, Issue 3, Cichester, UK: John Wiley \& Sons Ltd. 2004

10 Silagy C, Stead LF. Physician advice for smoking cessation (Cochrane Review). In: The Cochrane Library, Issue 1, Cichester, UK: John Wiley \& Sons Ltd. 2004

11 Stead LF, Lancaster T, Perera R. Telephone counselling for smoking cessation (Cochrane Review). In: The Cochrane Library, Issue 1, Cichester, UK: John Wiley \& Sons Ltd. 2004

12 Stead LF, Lancaster T. Group behaviour therapy programmes for smoking cessation (Cochrane Review). In: The Cochrane Library, Issue 3. Cichester, UK: John Wiley \& Sons Ltd. 2004

13 Wagena EJ, de Graaf L, Chavannes $\mathrm{NH}$, et al. Onrust over de veiligheid van bupropion als middel om te stoppen met roken onterecht. [Uneasiness about the safety of bupropion as an aid to smoking cessation unjustified.]. Ned Tijdschr Geneeskd 2001; 2001:31

14 Kwan AL, Meiners AP, van Grootheest AC, et al. Risico op convulsies bij gebruik van bupropion als hulpmiddel bij het stoppen met roken. [Risk of convulsions due to the use of bupropion as an aid for smoking cessation.]. Ned Tijdschr Geneeskd 2001; 145:277-278

15 Wagena EJ, Knipschild P, Zeegers MPA. Should nortriptyline be used as a first-line aid to help smokers quit? Results from a systematic review and meta-analysis. Addiction 2005; 100:317-326

16 Fiore MC, Bailey WC, Cohen SJ, et al. Treating tobacco use and dependence. Clinical Practical Guideline. Rockville, MD: US Department of Health and Human Services, 2000

17 Rice VH, Stead LF, Lancaster T. Nursing interventions for smoking cessation (Cochrane Review). In: The Cochrane Library, Issue 3. Cichester, UK: John Wiley \& Sons Ltd. 2004

18 Kwaliteitsinstituut voor de Gezondheidszorg CBO. Richtlijn Behandeling van Tabaksverslaving. [Guideline Treating Tobacco Dependence.]. Utrecht: Kwaliteitsinstituut voor de Gezondheidszorg CBO, 2004

19 Adriaanse HP, van Reek J, Rabier JR. Rookgedrag van Nederlandse huisartsen in de periode 1983-1988. [Smoking behaviour of Dutch family physicians in 1983-1988]. Ned Tijdschr Geneeskd 1990; 134:1502-1505

20 Van Reek J, Adriaanse H. Smoking by physicians in Scandinavia: 1952-1989. Scand J Soc Med 1991; 19:256-259

21 Pederson LL. Compliance with physician advice to quit smoking: a review of the literature. Prev Med $1982 ; 11: 71-84$

22 Smoking and health: physician responsibility. A statement of the Joint Committee on Smoking and Health. American College of Chest Physicians. American Thoracic Society. Asia Pacific Society of Respirology. Canadian Thoracic Society. European Respiratory Society, and International Union Against Tuberculosis and Lung Disease. Chest 1995; 108:1118-1121

23 Barengo NC, Sandstrom PH, Jormanainen VJ, et al. Changes in smoking prevalence among Finnish physicians 1990-2001. Eur J Public Health 2004; 14:201-203 
Chapter 2

24 John U, Hanke M. Tobacco-smoking prevalence among physicians and nurses in countries with different tobacco-control activities. Eur J Cancer Prev 2003; 12:235-237

25 Ohida T, Sakurai H, Mochizuki Y, et al. Smoking prevalence and attitudes toward smoking among Japanese physicians. JAMA 2001; 285:2643-2648 


\title{
Chapter 3
}

\section{Smoking cessation treatment by Dutch respiratory nurses: reported practice, attitudes and perceived effectiveness}

\author{
Daniel Kotz ${ }^{1}$ \\ Walter van Litsenburg ${ }^{2,4}$ \\ Raymond van Duurling ${ }^{3,4}$ \\ Onno C.P. van Schayck ${ }^{1}$ \\ Geertjan Wesseling ${ }^{3}$
}

Published in: Patient Education and Counseling 2008; 70: 40-49

${ }^{1}$ Department of General Practice, School for Public Health and Primary Care (CAPHRI), Maastricht University Medical Centre, Maastricht, the Netherlands. ${ }^{2}$ Catharina Hospital, Eindhoven, the Netherlands. ${ }^{3}$ Department of Respiratory Medicine, Maastricht University Medical Centre, Maastricht, the Netherlands. ${ }^{4}$ Dutch Society of Respiratory Nurses (NVL), Nijkerk, the Netherlands 


\section{ABSTRACT}

\section{Objectives}

To describe Dutch respiratory nurses' current smoking cessation practices, attitudes and beliefs, and to compare these with a survey from the year 2000, before the national introduction of a protocol for the treatment of nicotine and tobacco addiction (the L-MIS protocol).

\section{Methods}

Questionnaire survey among all 413 registered respiratory nurses in the Netherlands in 2006.

\section{Results}

The response rate was $62 \%$. Seventy-seven percent of the respondents reported to have "fairly good" or "good" knowledge of all steps of the L-MIS protocol. Seven out of ten behavioural techniques for smoking cessation from the protocol were used by more than $94 \%$ of the respondents. Seventy-four percent of the respiratory nurses recommended the use of either nicotine replacement therapy $(70 \%)$ or bupropion (44\%). Almost two thirds (65\% of 254 ) perceived lack of patient's motivation as the most important barrier for smoking cessation treatment; a fourfold increase compared to the year 2000.

\section{Conclusion}

We conclude that respiratory nurses are compliant with the L-MIS protocol. They offer intensive support and use behavioural techniques for smoking cessation more frequently than evidence-based pharmacological aids for smoking cessation. Perceived lack of patient's motivation forms the most important threat to respiratory nurses' future smoking cessation activities.

\section{Practice implications}

International guidelines acknowledge that respiratory patients have a more urgent need to stop smoking but have more difficulty doing so. They should be offered the most intensive smoking cessation counselling in combination with pharmacotherapy. This kind of counselling may be more feasible for respiratory nurses than for physicians who often lack time. Their efforts could be increased by reimbursing pharmacological aids for smoking cessation and by developing simple tools to systematically assess motivation to quit and psychiatric co-morbidity in smoking patients. 


\section{INTRODUCTION}

According to national and international guidelines ${ }^{2,3}$, nurses can play an important role with regard to smoking cessation treatment. They are the largest group among health care providers and can treat patients for smoking cessation effectively. ${ }^{4}$ In the Netherlands, as in a number of other countries, respiratory nurses are a relatively new discipline (starting in the 1990's). They are nurses with additional training in respiratory medicine. Supporting smoking cessation attempts is a vital part of respiratory nurses' work as most of their clientele are patients with diseases closely related to smoking. For example, smoking is the primary cause of chronic obstructive pulmonary disease (COPD), and smoking cessation is the single most effective way to reduce the risk of developing COPD and to affect the outcome in patients at all stages of the disease. ${ }^{5,6}$

There are only little empirical data available on Dutch respiratory nurses' reported practice, attitudes and beliefs towards smoking and smoking cessation. The only study we could find is a report on a survey that was performed in 2000 by a research and consultancy agency on behalf of the Dutch foundation for a smoke free future (STIVORO). ${ }^{7}$ Since then, a protocol for the treatment of nicotine and tobacco addiction (the so-called "L-MIS") ${ }^{1}$ has been implemented nationally among all respiratory nurses and new evidence has become available on various smoking cessation strategies. $^{8-11}$ The L-MIS protocol basically describes seven steps of smoking cessation treatment during several consultations. These steps are listed in the text box.

We performed a questionnaire survey among all registered respiratory nurses in the Netherlands to assess and describe their current smoking cessation practices, attitudes and beliefs. Furthermore, we wanted to compare the results from the current survey with the STIVORO survey from the year 2000, before the introduction of the L-MIS protocol. By doing these analyses, we wanted to assess the feasibility of respiratory nurses for taking on greater responsibility for smoking cessation treatment, because primary care physicians can only deliver minimal care in this respect.

\section{METHODS}

\section{Questionnaire development and content}

The questionnaire was developed by the author and the co-authors (a group of two epidemiologists, one lung physician, and two respiratory nurses). The relevance of the different aspects of the questionnaire (content validity) was assessed by reviewing the content of current evidence-based guidelines ${ }^{2,3}$ and earlier surveys among Dutch respiratory nurses ${ }^{7}$ and physicians ${ }^{12}$. Furthermore, we asked experts in the field to judge the questionnaire (face validity). The questionnaire included questions 
about current practice, attitudes, knowledge, skills, perceived effectiveness and barriers with regard to the treatment of smoking. We asked two questions for defining the respondents' own smoking status: (1) "Do you smoke now and again or daily?", and (2) "Did you smoke in the past now and again or daily?". A respondent who answered "yes" to question 1 was defined as "current smoker", a respondent who answered "no" to question 1 but "yes" to question 2 was defined as "former smoker", and a respondent who answered "no" to both questions was defined as "never smoker". The questionnaire was pre-tested among four respiratory nurses from Maastricht University Hospital and subsequently adjusted. The final questionnaire comprised a booklet of 10 black and white pages (DIN A4) with orderly and well-structured text. The completion time was approximately 20 minutes.

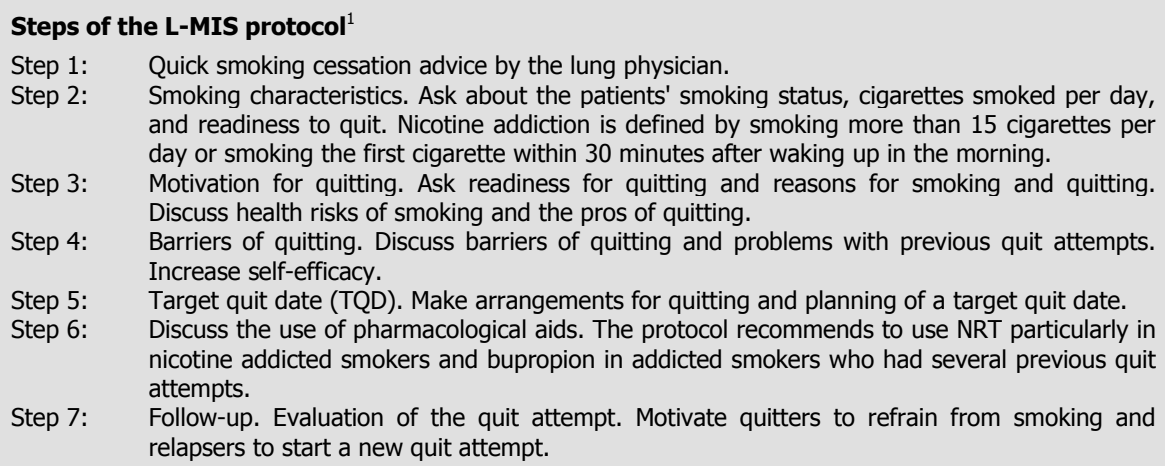
and readiness to quit. Nicotine addiction is defined by smoking more than 15 cigarettes per day or smoking the first cigarette within 30 minutes after waking up in the morning.

Step 3: Motivation for quitting. Ask readiness for quitting and reasons for smoking and quitting. Discuss health risks of smoking and the pros of quitting.

Step 4: $\quad$ Barriers of quitting. Discuss barriers of quitting and problems with previous quit attempts. Increase self-efficacy.

Step 5: $\quad$ Target quit date (TQD). Make arrangements for quitting and planning of a target quit date.

Step 6: Discuss the use of pharmacological aids. The protocol recommends to use NRT particularly in nicotine addicted smokers and bupropion in addicted smokers who had several previous quit attempts.

Step 7: $\quad$ Follow-up. Evaluation of the quit attempt. Motivate quitters to refrain from smoking and relapsers to start a new quit attempt.

Steps 2 to 6 occur during the first and second consultation (two weeks later) with the respiratory nurse. The duration of both consultations is $30-45$ minutes. A consultation by phone is planned on the TQD. Follow-up consultations are recommended two weeks after the TQD and after three, six, and twelve months. (5-10 minutes each).

\section{Questionnaire administration}

The survey was carried out in cooperation with The Dutch Society of Respiratory Nurses (NVL). New members of the NVL are routinely asked written consent to be approached by third parties. Approximately $90 \%$ of new members usually give their consent. The NVL provided us with an electronic file with the names and postal addresses of these members $(\mathrm{N}=413)$. The survey was announced on the society's website and in their bulletin. We sent the first mailing in June 2006, and two reminders including new copies of the original questionnaire to all non-respondents in July and August 2006. We sent a reply card along with the last mailing on which persistent non-respondents could report reasons for not responding. Every mailing was introduced with a personalized letter stressing the importance of the topic and signed by the chair of the NVL (WL) and a lung physician (GW) who is well-known in the field. Also, we announced to give away 40 bottles of red wine (Château Moulin 2004) among a random sample of respondents. Respondents could use pre-paid return envelopes to return the questionnaire. 


\section{Data analysis}

The design of the study is cross-sectional. In order to assess developments during the past years, we compared our results where possible with the survey performed on behalf of STIVORO in the year $2000 .^{7}$ That survey assessed the smoking cessation strategies among all members of the NVL at that time $(\mathrm{N}=288)$. One hundred forty-one (54\%) filled out and returned the questionnaire. Unfortunately, results from this survey have not been published in an internationally accessible journal but in an internal report. We have approval from STIVORO to use these results.

We used 4- or 5-point Likert scales for questions measuring the frequency of a behaviour or the degree of agreement with a certain statement. We dichotomized these questions afterwards. This is further explained for each question in the results section of this article. We did not calculate reliability scores because we did not construct sum scales for measuring specific concepts but rather used separate questions. We omitted missing values on variables when calculating percentages (indicated as "valid" percentages in the tables). The proportion of missing values was on average between 0 and $2 \%$ for each variable. All analyses were performed using the SPSS (version 13.0).

\section{RESULTS}

Two hundred fifty-four of the 413 respiratory nurses filled out and returned the questionnaire (response rate $62 \%$ ). Another $46(11 \%)$ returned the reply card of the last mailing and stated reasons for not responding. The most frequently mentioned reasons were "I only work with children", "I do not work as a respiratory nurse any more", and "I do not have to do with smoking patients in my job".

\section{Characteristics of the study population}

Table 1 shows demographic, smoking and work-related characteristics of the respondents. Two hundred respiratory nurses were female (95\% of 254), and the mean age was 44 years. Thirteen respiratory nurses were current smokers (5\%); a lower proportion than among Dutch general practitioners $(8 \%)$, and similar to Dutch cardiologists and lung physicians (both 4\%). ${ }^{12}$ Most respondents were registered nurses with either higher $(51 \%)$ or intermediate $(19 \%)$ vocational education. This educational level is similar to that of a bachelor. Of the respondents who had additional training in respiratory nursing (93\%), the majority had this training at the national training centre SSSV (Stichting Specifieke Scholing Verpleegkundigen). The centre offers a 6-month-training course and examination on nursing of patients with asthma and COPD. Respiratory nurses generally spend approximately $50 \%$ of their time on direct patient contact, $20 \%$ on education, and the remainder on innovation, 
research, and consultation. Most respiratory nurses from this survey worked either in a district hospital (53\%) and/or in home care (34\%).

The demographic characteristics of the respondents from the current survey were comparable with the respondents from the 2000 survey: $94 \%$ of the respondents from that survey were female, and the mean age was 41 years. Also the work setting was comparable: $50 \%$ of the respondents from the 2000 survey worked in a district hospital, and $37 \%$ in home care. The proportion of current smokers, however, was higher in the year 2000: $9 \%$.

Ninety-six percent of the respondents from the current survey were familiar with the L-MIS protocol for the treatment of smoking cessation in lung patients ${ }^{1}$, which had been implemented nationally among all respiratory nurses between the years 2000 and 2006. Seventy-seven percent reported to have "fairly good" or "good" knowledge of all steps of the protocol.

\section{Intensity of smoking cessation support}

Two hundred and five respiratory nurses reported to treat patients for smoking cessation during consulting hours (the remaining 49 reported not to treat patients for smoking cessation at all). This subgroup reported to have a median number of 6 consultations with the same patient for smoking cessation. The mean time spent was 36 minutes for the first consultation, 23 minutes for the second consultation, and 18 minutes for any follow-up consultation. This is more intensive smoking cessation support compared with data from the 2000 survey. Half of the respondents from that survey reported to have more than one consultation per patient (mean number of 4 consultations within this subgroup). The mean time spent was 19, 17, and 14 minutes per consultation respectively.

\section{Assessing and confronting the smoking patient}

Of the 205 respiratory nurses that reported to treat patients for smoking cessation, 166 ( $81 \%$ of 205 ) reported to systematically assess the motivation of the patient to quit smoking. Seventy-three (37\%) used a validated questionnaire for this (which questionnaire was not stated). One hundred twenty-two respiratory nurses $(60 \%)$ reported to systematically assess the degree of tobacco addiction of the patient, 30 $(15 \%)$ by using the Fagerström Test for Nicotine Dependence ${ }^{13}$. Seventy-six respiratory nurses (37\%) reported to systematically assess signs and symptoms of psychiatric co-morbidity such as depression or anxiety. Only one person used a validated questionnaire: the Beck Depression Inventory ${ }^{14}$.

One hundred eighty-nine respiratory nurses (92\% of 205) reported to use the "Fletcher curve"15 and $143(70 \%)$ to use results from spirometry for confronting the patient with the harmful effects of smoking during smoking cessation consultations. 
Within the latter group, 74 (52\% of 143 ) believed that confrontation with spirometry for smoking cessation is more effective than health education without confrontation.

\section{Use of behavioural techniques and recommendation of aids for smoking cessation}

Figure 1 and figure 2 show the use of various behavioural techniques and aids for smoking cessation. The black bars represent the proportion of respiratory nurses from this 2006 survey reporting to use the specific technique or aid "often" or "always" (versus "sometimes" or "never"). Responses are restricted to the sub-sample of 205 respiratory nurses who reported to treat patients for smoking cessation. The grey bars represent the response of respiratory nurses from the 2000 survey.

Figure 1 shows that, compared with the survey in 2000, all ten behavioural techniques for smoking cessation were used by a higher proportion of respiratory nurses in 2006 (on average 93\% compared to $72 \%$ in 2000). The largest increases were found in making arrangements for quitting (83\% compared to $38 \%$ ) and setting a target quit date (79\% compared to $32 \%$ ). Seven out of ten techniques were used by more than $94 \%$ of the respondents. Seventy-four percent of the respiratory nurses recommended the use of the two most important evidence-based pharmacological aids for smoking cessation at that time; either nicotine replacement therapy (NRT, $70 \%$ ) or bupropion (44\%). Notriptyline, a tricyclic anti-depressant which has also been shown to be an effective aid for smoking cessation ${ }^{8}$, was recommended by $19 \%$. Figure 2 shows that the proportion of respiratory nurses recommending NRT and bupropion was similar in 2006 compared with 2000. There was a sharp increase of nurses recommending individual counselling for smoking cessation. Furthermore, approximately $20 \%$ recommended acupuncture or laser therapy and $6 \%$ hypnosis therapies not proven to be effective.

\section{Perceived self-efficacy towards smoking cessation}

We asked the respiratory nurses about their perceived effectiveness towards strategies for smoking cessation. Seventy-two percent $(n=184$ of 254$)$ of the respondents agreed that their behavioural counselling helps smokers to quit smoking. Seventy percent $(n=178)$ agreed that NRT, such as nicotine gum or patch, helps smokers to quit smoking and $65 \%(n=164)$ agreed about anti-depressants, such as nortriptyline or bupropion. Forty-two percent $(n=107)$ agreed that alternative treatments, such as acupuncture, help smokers to quit smoking. We also asked respiratory nurses to estimate what proportion of smokers that receive smoking cessation treatment remains abstinent for at least one year. The median percentage estimated was $30 \%$ (range $0 \%$ to $90 \%$ ).

One hundred-ten out of 254 respiratory nurses (44\%) believed that a smoker with asthma or COPD has more difficulties to quit smoking than a "healthy" smoker. We 
asked an open-ended question to explain why. The most frequently given explanations were that smokers with asthma or COPD have a longer smoking history and a higher degree of nicotine addiction, more psycho-social co-morbidity, and that they perceive the cigarette as their last and only solace.

Figure 3 shows seven potential barriers for the treatment of smoking cessation. The bars represent the proportion of respiratory nurses who perceive to be hindered by the specific barrier "to a considerable" or "large extent" when treating a patient (versus "to a little extent", "not at all", or "indecisive"). Whereas six barriers were perceived by a minority of respiratory nurses (by less than a quarter), almost two thirds (65\% of 254 ) perceived lack of patient's motivation as a barrier for smoking cessation treatment. This is almost four times as much as in the 2000 survey where $17 \%$ reported lack of patient's motivation as a barrier. In that year, lack of time $(31 \%)$, skills $(29 \%)$, and knowledge $(26 \%)$ where the most frequently reported barriers.

\section{Perspectives towards a smoke-free future}

At the end of the questionnaire, we asked an open ended question about the respiratory nurses' personal view how smoking cessation should be further stimulated in the Netherlands. The three most frequently offered suggestions were: the introduction of a smoking ban in bars and restaurants, increased health education and promotion among the youth, and reimbursement of smoking cessation aids.

\section{DISCUSSION AND CONCLUSION}

We conducted a questionnaire survey about smoking cessation treatment among all respiratory nurses registered at the Dutch Society of Respiratory Nurses (NVL). We compared our results where possible with the results from a survey conducted on behalf of the Dutch foundation for a smoke free future (STIVORO) in 2000, before the national introduction of a protocol for the treatment of nicotine and tobacco addiction (the so-called "L-MIS" protocol). Compared with the year 2000, respiratory nurses nowadays offer more intensive smoking cessation treatment to patients. A higher proportion uses various behavioural techniques which are important for smoking cessation whereas the use of pharmacological aids for smoking cessation has not increased (except for nortriptyline). A high proportion of respiratory nurses feels confident about the effectiveness of their behavioural counselling, NRT, and anti-depressants for smoking cessation. The most important barrier for smoking cessation treatment, currently perceived by two thirds of the respondents, is lack of patient's motivation. 
Our findings are based on a repeated survey and not on a controlled trial, and therefore, no firm conclusions can be drawn about the effects of the implementation of the L-MIS protocol between 2000 and 2006. Despite this, we think that the results show that the majority of the respiratory nurses (77\%) are familiar with all steps of the L-MIS protocol and suggest a very high compliance with the protocol. The recommendations from the protocol also seem to explain why bupropion is used less frequently than NRT and why both aids are used by not more than $44-70 \%$ of the respiratory nurses. Previous research has shown that the combination of counselling and pharmacotherapy is more effective than either alone ${ }^{8,9,16}$, and international guidelines recommend the use of pharmacotherapy in all patients trying to make a quit attempt. ${ }^{3,17}$ Therefore, respiratory nurses should be encouraged to recommend more frequently evidence-based pharmacological aids for smoking cessation in combination with counselling. Today, smoking cessation aids are not reimbursed in the Netherlands, and the high costs of these aids, especially bupropion (Zyban ${ }^{\mathrm{TM}}$, EUR 70,- per month of treatment ${ }^{18}$ ), restrain many smokers from their use. Nortriptyline, a generic non-patent medicine, reimbursed by all health-care insurance companies, has been shown to be a cheap and effective alternative..$^{8,19}$

The patient's 12-month quit rate estimated by respiratory nurses from our survey (median $30 \%$ ) seems to be low but is slightly optimistic compared to success rates reported from cotinine-validated explanatory randomized controlled trials (approximately $18-30 \%$ for a combination of pharmacotherapy with counselling). ${ }^{3}$ Half of the respiratory nurses believe that smokers with asthma or COPD have more difficulties quitting than a "healthy" smoker, an impression also shared by the European Respiratory Society's task force on smoking cessation in patients with respiratory diseases. ${ }^{17}$ The nurses suspect addiction and psycho-social co-morbidity to be reasons for these difficulties. In fact, studies show that smokers with COPD tend to be more addicted to nicotine and tobacco ${ }^{20}$ and are more likely to have depression ${ }^{21}$ and anxiety $^{22}$. As psycho-social co-morbidity is prevalent in smokers with COPD and associated with their smoking behaviour and attempts to quit smoking, systematic assessment of mental health and use of appropriate counselling techniques are important. Only a minority of respiratory nurses (37\%) currently assesses psychosocial problems and most of them do not do this systematically or with the use of validated instruments.

Respiratory nurses perceive lack of patient's motivation as their most important barrier for smoking cessation treatment. Motivation to quit is an important condition for successful smoking cessation, but there is no good, validated measure for assessing the degree of motivation to date. ${ }^{17}$ However, is it really a lack of motivation or rather low perceived self-efficacy towards quitting and frustration about numerous relapses that discourage smokers to start a new quit attempt? Probably the majority of smokers would give up smoking if it were cheap and easy. An explanation for the 
fourfold increase in proportion of respiratory nurses who perceive this barrier in 2006 compared to 2000 might be that the patients they see nowadays who still smoke represent a group of "die-hards" who have enormous difficulties to give up smoking. Respiratory nurses' perceptions of patients' low motivation and high relapse rates (70\% after one year) are likely to influence their attitudes towards smoking cessation. Twenty-two percent of respondents from the current survey stated: "I find it ridiculous that lung patients smoke despite their disease" (data not shown in the results section). As a dangerous consequence, negative attitudes might undermine respiratory nurses' future smoking cessation activities.

A different barrier for taking action against smoking may be personal smoking habits. The results from our survey show that the prevalence of smoking among respiratory nurses is only $5 \%$, which is lower than in Dutch GPs $(8 \%)^{12}$ and much lower than in the general population in the Netherlands $(30 \%)^{23}$. This indicates, that personal smoking may be a barrier only for a very small group of respiratory nurses.

The most frequently offered suggestions to promote smoking cessation in the Netherlands were the introduction of a smoking ban in bars and restaurants, increased health education and promotion among the youth, and reimbursement of smoking cessation aids. The first two mentioned suggestions are well-known measures to prevent smoking, but are out of respiratory nurses' influence. Reimbursement of smoking cessation aids, however, has been shown to be effective in promoting smoking cessation, both internationally ${ }^{24}$ as in the Netherlands. ${ }^{25}$ Reimbursement of evidence-based pharmacological aids has the potential to increase respiratory nurses' efficacy in smoking cessation treatment.

The findings from this questionnaire survey are limited by the moderate response rate of $62 \%$. Such a response rate is not unusual for studies in this field. The response to our survey was higher than to the previous survey among respiratory nurses from the year 2000 (54\%). Recent surveys about smoking cessation practices conducted among Dutch general practitioners, cardiologists and lung physicians in the years 2002 and 2003 yielded response rates of 42-69\%. ${ }^{12}$ Eleven percent of our population indicated by using a reply card that the content of the questionnaire was not applicable to them. We do not have information about the remaining $23 \%$, but if selection bias has occurred, it is likely that the responders represent a group of respiratory nurses that is more active in smoking cessation treatment than the nonresponders. In that case, the smoking cessation activities reported in this survey may be an overestimation of the actual situation. The comparison of the results from the current survey with data from the 2000 survey is limited by the fact that this is not a repeated survey among the same population. The characteristics of respondents from the two surveys were comparable, except for the proportion current smokers, 
which was lower in 2006 (5\%) than in 2000 (9\%). It cannot be ruled out that a different selection of respondents has biased the comparison of results.

\section{Conclusion}

Based on the results of our national survey, we conclude that respiratory nurses are compliant with the protocol for smoking cessation practices in lung patients which has been implemented nationally in previous years. They offer intensive support and use behavioural techniques for smoking cessation more frequently than evidencebased pharmacological aids for smoking cessation. By delivering such care for smoking cessation, respiratory nurses play a key role in the treatment of respiratory patients who smoke. Perceived lack of patient's motivation forms the most important threat to respiratory nurses' future smoking cessation activities.

\section{Practice implications}

International guidelines acknowledge that respiratory patients have a more urgent need to stop smoking but have more difficulty doing so than the average smoker. ${ }^{17}$ Tobacco dependence is a chronic condition that often requires repeated intervention and the effectiveness of interventions increase with treatment intensity. ${ }^{3}$ Respiratory patients should be offered the most intensive smoking cessation intervention ${ }^{26}$, a combination of intensive smoking cessation counselling in combination with pharmacotherapy. Respiratory nurses are key to the delivery of smoking cessation treatment according to the Global Initiative for Chronic Obstructive Lung Disease (GOLD) guidelines. ${ }^{26}$ The results from our survey show that respiratory nurses are able to deliver this treatment on the basis of the national protocol for smoking cessation. Intensive smoking cessation counselling may be more feasible for respiratory nurses than for physicians who often lack time. Their efforts could be increased by reimbursing pharmacological aids for smoking cessation and by developing simple tools to systematically assess motivation to quit and psychiatric co-morbidity in smoking patients. 
Table 1: Demographic, smoking and work-related characteristics of the study population

\begin{tabular}{|c|c|}
\hline Characteristics & Respiratory nurses $(n=254)$ \\
\hline Female sex, $\mathrm{n}$ (valid percentage) & $240(94.9)$ \\
\hline Years of age, mean (SD) & $44.3(8.1)$ \\
\hline \multicolumn{2}{|l|}{ Smoking status, $\mathrm{n}$ (valid percentage) } \\
\hline current smoker & $13(5.2)$ \\
\hline former smoker & $127(51.0)$ \\
\hline never smoker & $109(43.8)$ \\
\hline \multicolumn{2}{|l|}{ Education, $\mathrm{n}$ (valid percentage) } \\
\hline registered nurse (higher vocational education) & $127(51.0)$ \\
\hline nurse (intermediate vocational education) & $47(18.9)$ \\
\hline nurse practitioner & $4(1.6)$ \\
\hline nursing specialist & $31(12.4)$ \\
\hline other or more than one answer & $40(16.0)$ \\
\hline Additional training in respiratory nursing, $\mathrm{n}$ (valid percentage) & $234(92.9)$ \\
\hline Years of working experience as respiratory nurse, mean (SD) & $6.7(4.9)$ \\
\hline Hours a week working as respiratory nurse, mean (SD) & $20.7(9.1)$ \\
\hline \multicolumn{2}{|l|}{ Work setting $^{\dagger}, \mathrm{n}$ (valid percentage) } \\
\hline district hospital & $134(52.8)$ \\
\hline home care & $85(33.5)$ \\
\hline general practice & $46(18.1)$ \\
\hline university hospital & $19(7.5)$ \\
\hline other & $26(6.7)$ \\
\hline
\end{tabular}

${ }^{\dagger}$ multiple responses possible

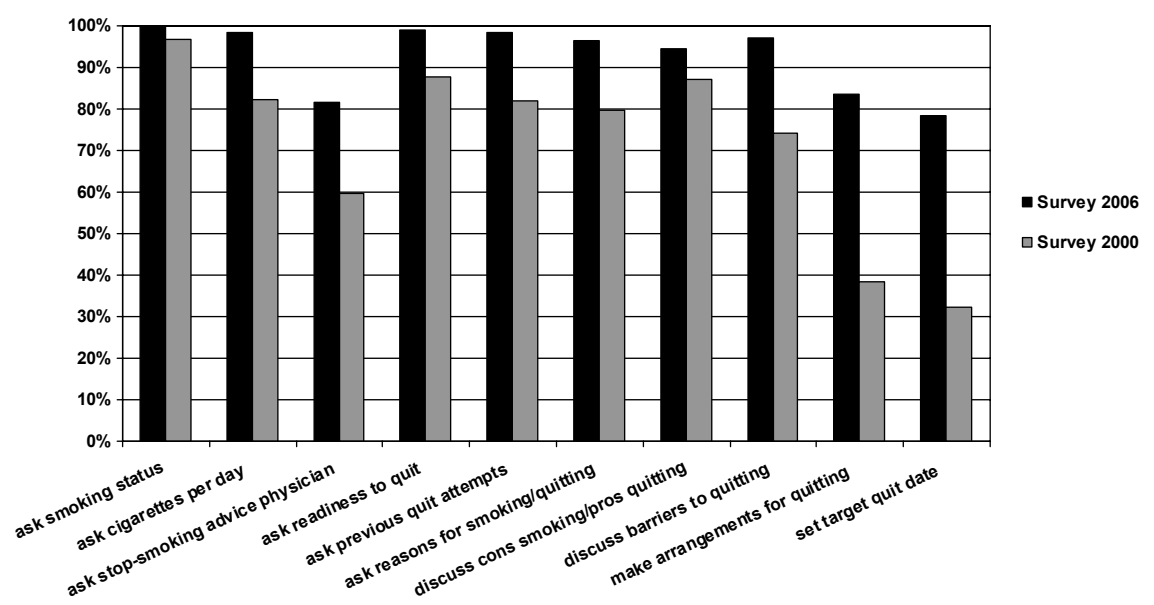

Figure 1: Proportion of respiratory nurses reporting to use behavioural techniques for smoking cessation. Black columns: results from the current 2006 survey, grey columns: results from the 2000 survey. 


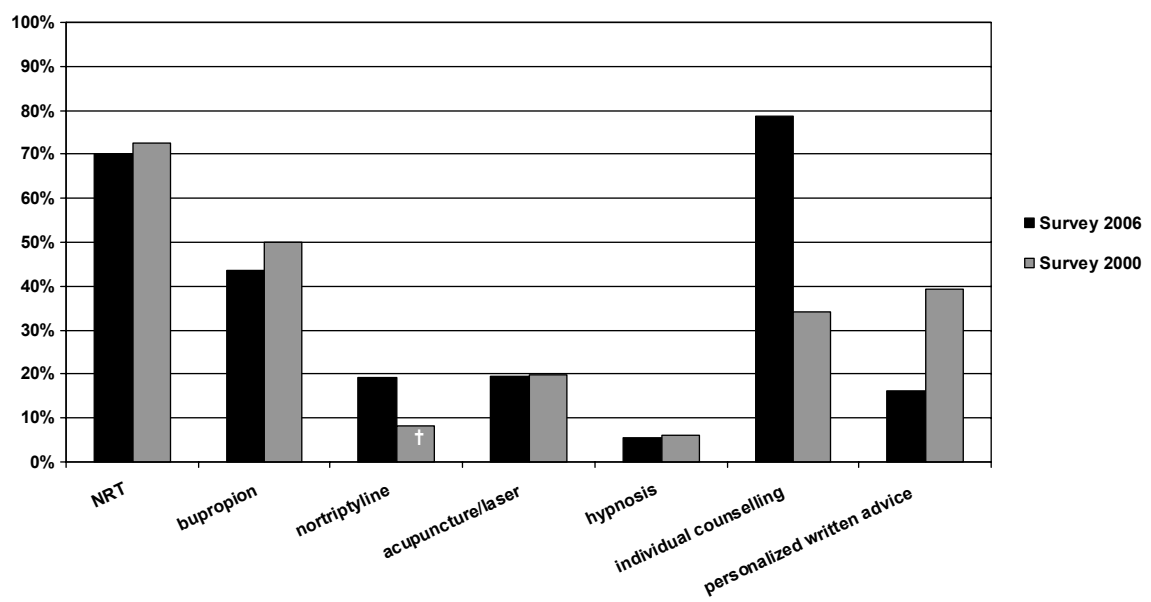

Figure 2: Proportion of respiratory nurses reporting to recommend the use of smoking cessation aids. Black columns: results from the current 2006 survey, grey columns: results from the 2000 survey. ${ }^{\dagger}$ Tranquillizer or anti-depressant (not specifically nortriptyline). NRT = nicotine replacement therapy.

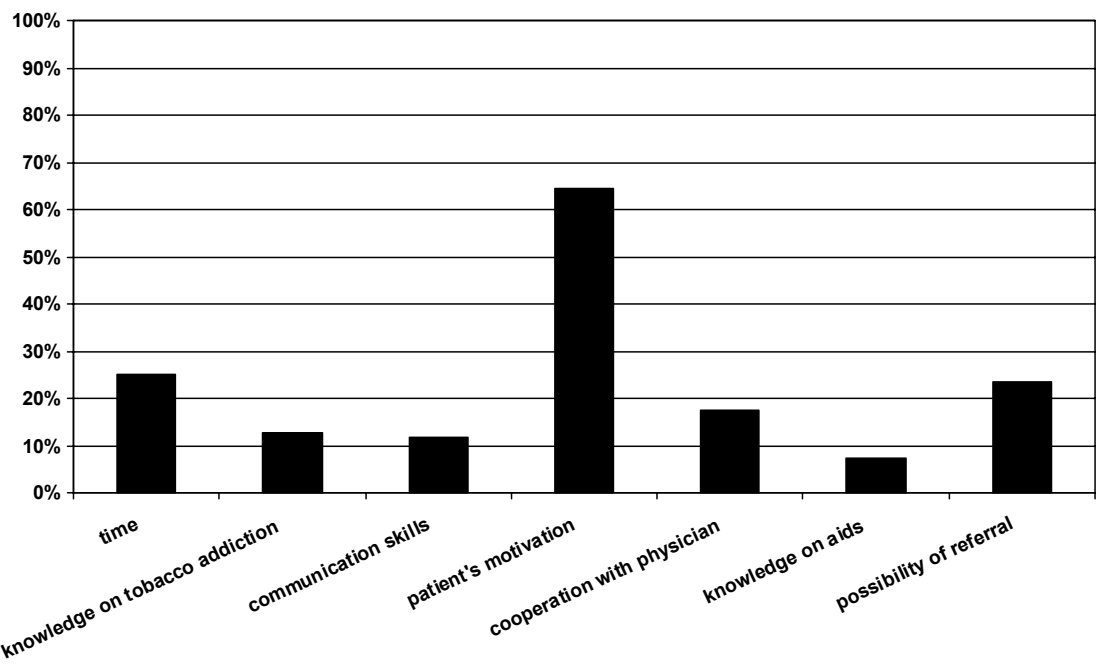

Figure 3: Proportion of respiratory nurses reporting barriers for smoking cessation treatment. 


\section{APPENDIX}

\section{Sample of questions from the questionnaire}

The following questions refer to the L-MIS.

Do you know what the L-MIS is?

Are you familiar with all the steps of the L-MIS?

not at all a little moderately fairly good good

$\begin{array}{lllll}\square & \square & \square & \square & \square\end{array}$

I help smoking lung patients during consultations. ..

... always with smoking cessation.

... most of the time with smoking cessation.

... in approximately half of the cases with smoking cessation

... sometimes with smoking cessation.

... never with smoking cessation.

How often do you on average have contact with the same patient about smoking cessation? number: I__ $_{\text {I }}$ times with the same patient

How many minutes do you on average spend per consultation on smoking behaviour or smoking cessation? first consultation: number: I_l___ minutes second consultation: number: I_L___ minutes third consultation: number: _____ minutes

(fill in " 0 " in case you do not plan the specific consultation)

Do you try to systematically assess whether the patient is inner self motivated to quit smoking?

$\square$ yes $\rightarrow$ if yes, how do you do that?

$\square$ by using a valid questionnaire

$\square$ otherwise, namely:

no

Do you systematically assess the degree of nicotine dependence of the patient?

$\square$ yes $\rightarrow$ if yes, how do you do that?

$\square$ by using the Fagerström Test of Nicotine Dependence (FTND)

$\square \quad$ no

otherwise, namely:

Do you systematically look for signs of psychiatric disorders (co-morbidity) in a smoking patient, such as depression or anxiety?

$\square$ yes $\rightarrow$ if yes, how do you do that?

$\square$ by using a valid questionnaire such as the Beck Depression Inventory (BDI)

no

$\square$ by using a valid questir
$\square$ otherwise, namely: 
Do you now and again use the Fletcher curve to confront the smoker with the harmful effects of smoking?

$\square$ yes

$\square \quad$ no

$\square \quad$ I do not know the Fletcher curve

Listed below are aspects of health education for smoking cessation. How often do you use these techniques?

I ask patients if they smoke.

I ask patients how many cigarettes per day they smoke.

I ask patients if they have received stop-smoking advice from their physician.

I ask patients if they are thinking about quitting smoking. I ask patients if they have made previous quit attempts.

I ask patients about their reasons for willing or not willing to quit smoking.

I discuss pros and cons of quitting smoking with patients. I discuss barriers to quitting smoking with patients.

I make arrangements for quitting smoking with patients.

I set a target quit date with patients.

$\begin{array}{cccc}\text { never } & \text { sometimes } & \text { often } & \text { always } \\ \square & \square & \square & \square \\ \square & \square & \square & \square \\ \square & \square & \square & \square\end{array}$

$\begin{array}{llll}\square & \square & \square & \square \\ \square & \square & \square & \square \\ \square & \square & \square & \square \\ \square & \square & \square & \square \\ \square & \square & \square & \square \\ \square & \square & \square & \square \\ \square & \square & \square & \square\end{array}$

Listed below are aids for smoking cessation. How often do you give information about these aids or recommend their use?

Nicotine gum

Nicotine patch

Nicotine inhaler

Nicotine lozenge

Bupropion (Zyban ${ }^{\mathrm{TM}}$ )

Nortriptyline (Notrilen ${ }^{\circledR}$ )

Personalized written advice

Individual counselling

Laser therapy

Acupuncture

Hypnosis

never sometimes often always

Listed below are four statements about the efficacy of smoking cessation aids. Please answer whether you agree with these statements.

The behavioural counselling I offer helps smokers

$\begin{array}{cc}\text { totally } & \text { indecisive/ } \\ \text { disagree } & \text { disagree } \\ \text { don't know }\end{array}$

agree totally agree

to quit smoking.

Nicotine replacement therapy (such as nicotine gum or nicotine patch) helps smokers to quit

smoking.

Anti-depressants (such as bupropion [Zyban ${ }^{\mathrm{TM}}$ ] or nortriptyline [Nortrilen ${ }^{\circledR}$ ] help smokers to quit smoking.

Alternative treatments (such as acupuncture) help smokers to quit smoking. 
Do you think a smoker with asthma or COPD has more difficulties to quit smoking than a "healthy" smoker?

$\square$ yes $\rightarrow$ if yes, why do you think so?

no

To what degree do you feel that the aspects listed below hinder you when treating tobacco addiction?

\begin{tabular}{|c|c|c|c|c|c|}
\hline & $\begin{array}{l}\text { not } \\
\text { at all }\end{array}$ & a little & $\begin{array}{l}\text { indeci- } \\
\text { sive/don't } \\
\text { know }\end{array}$ & $\begin{array}{c}\text { to a } \\
\text { considerable } \\
\text { extent }\end{array}$ & $\begin{array}{l}\text { to a large } \\
\text { extent }\end{array}$ \\
\hline Lack of time & $\square$ & $\square$ & $\square$ & $\square$ & $\square$ \\
\hline ack of knowledge on tobacco addiction & $\square$ & $\square$ & $\square$ & $\square$ & $\square$ \\
\hline $\begin{array}{l}\text { Lack of communication skills with regard to } \\
\text { smoking cessation counselling }\end{array}$ & $\square$ & $\square$ & $\square$ & $\square$ & $\square$ \\
\hline Lack of patient's motivation & $\square$ & $\square$ & $\square$ & $\square$ & $\square$ \\
\hline ack of cooperation with the GP or specialist & $\square$ & $\square$ & $\square$ & $\square$ & $\square$ \\
\hline ack of knowledge on smoking cessation aids & $\square$ & $\square$ & $\square$ & $\square$ & $\square$ \\
\hline Lack of possibilities for referral of patients & $\square$ & $\square$ & $\square$ & $\square$ & $\square$ \\
\hline
\end{tabular}




\section{REFERENCES}

1 STIVORO. De L-MIS: stoppen met roken voor longpatiënten. Handleiding voor longverpleegkundigen. [The L-MIS: smoking cessation in lung patients. Strategy for respiratory nurses]. Den Haag: STIVORO, 2003

2 Kwaliteitsinstituut voor de Gezondheidszorg CBO. Richtlijn Behandeling van Tabaksverslaving. [Guideline Treating Tobacco Dependence.]. Utrecht: Kwaliteitsinstituut voor de Gezondheidszorg CBO, 2004

3 Fiore MC, Bailey WC, Cohen SJ, et al. Treating tobacco use and dependence. Clinical Practical Guideline. Rockville, MD: US Department of Health and Human Services, 2000

4 Rice VH, Stead LF, Lancaster T. Nursing interventions for smoking cessation. Cochrane Database Syst Rev 2004

5 Anthonisen NR, Connett JE, Kiley JP, et al. Effects of smoking intervention and the use of an inhaled anticholinergic bronchodilator on the rate of decline of FEV1. The Lung Health Study. J Am Med Assoc 1994; 272:1497-1505

6 Pauwels RA, Buist AS, Calverley PM, et al. Global strategy for the diagnosis, management, and prevention of chronic obstructive pulmonary disease. NHLBI/WHO Global Initiative for Chronic Obstructive Lung Disease (GOLD) Workshop summary. Am J Respir Crit Care Med 2001; 163:1256-1276

7 de Weerdt I, Spapen S. Voorlichting over stoppen met roken door longverpleegkundigen. Inventariserend onderzoek naar de mogelijkheden voor toepassing van de L-MIS. [Health education for smoking cessation by respiratory nurses. Investigating research on the possibilities to apply the L-MIS.]. Haarlem: ResCon, research \& consultancy, 2000

8 Hughes JR, Stead LF, Lancaster T. Antidepressants for smoking cessation. Cochrane Database Syst Rev 2004

9 Lancaster T, Stead LF. Individual behavioural counselling for smoking cessation. Cochrane Database Syst Rev 2004

10 Silagy C. Physician advice for smoking cessation. Cochrane Database Syst Rev 2004

11 Willemsen MC, Wagena EJ, van Schayck CP. De effectiviteit van stoppen-met-rokenmethoden die in Nederland beschikbaar zijn: een systematische review op basis van Cochrane-gegevens.[The efficacy of smoking cessation methods available in the Netherlands: a systematic review based on Cochrane data.]. Ned Tijdschr Geneeskd 2003

12 Kotz D, Wagena EJ, Wesseling G. Smoking cessation practices of Dutch general practitioners, cardiologists, and lung physicians. Respiratory Medicine 2007; 101:568-573

13 Heatherton TF, Kozlowski LT, Frecker RC, et al. The Fagerström Test for Nicotine Dependence: a revision of the Fagerström Tolerance Questionnaire. Br J Addict 1991; 86:1119-1127

14 Beck AT, Ward $\mathrm{CH}$, Mendelson $\mathrm{M}$, et al. An inventory for measuring depression. Arch Gen Psychiatry $1961 ; 4: 561-571$

15 Fletcher C, Peto R. The natural history of chronic airflow obstruction. Brit Med J 1977; 1:1645-1648

16 Silagy C, Lancaster T, Stead LF, et al. Nicotine replacement therapy for smoking cessation. Cochrane Database Syst Rev 2004

17 Tonnesen P, Carrozzi L, Fagerstrom KO, et al. Smoking cessation in patients with respiratory diseases: a high priority, integral component of therapy. ERS task force guideline. Eur Respir J 2007; 29:390-417

18 College voor Zorgverzekeringen [Health Care Insurance Board]. Farmacotherapeutisch Kompas. [Pharmacotherapeutical compass], 2007

19 Wagena EJ, Knipschild P, Zeegers MPA. Should nortriptyline be used as a first-line aid to help smokers quit? Results from a systematic review and meta-analysis. Addiction 2005; 100:317-326

20 Jimenez-Ruiz CA, Masa F, Miravitlles M, et al. Smoking Characteristics : Differences in Attitudes and Dependence Between Healthy Smokers and Smokers With COPD. Chest 2001; 119:1365-1370

21 Borson S, Claypoole K, McDonald GJ. Depression and Chronic Obstructive Pulmonary Disease: Treatment Trials. Semin Clin Neuropsychiatry 1998; 3:115-130

22 Brenes GA. Anxiety and chronic obstructive pulmonary disease: prevalence, impact, and treatment. Psychosom Med 2003; 65:963-970

23 Centraal Bureau voor de Statistiek [Statistics Netherlands] (CBS). Gerapporteerde gezondheid en leefstijl 2006. [Reported health and life style 2006.]

24 Kaper J, Wagena EJ, Severens JL, et al. Healthcare financing systems for increasing the use of tobacco dependence treatment. Cochrane Database Syst Rev 2005 
25 Kaper J, Wagena EJ, Willemsen MC, et al. Reimbursement for smoking cessation treatment may double the abstinence rate: results of a randomized trial. Addiction 2005; 100:1012-1020

26 Global Initiative for Chronic Obstructive Lung Disease. Global strategy for the diagnosis, management, and prevention of chronic pulmonary disease. Executive summary (http://www.goldcopd.com): NHLBI / WHO, 2006 
MAIN PART 



\title{
Chapter 4
}

\section{Principles of confrontational counselling in smokers with chronic obstructive pulmonary disease (COPD)}

\author{
Daniel Kotz ${ }^{1}$ \\ Marcus J.H. Huibers ${ }^{2}$ \\ Rein Vos $^{3}$ \\ Onno C.P. van Schayck ${ }^{1}$ \\ Geertjan Wesseling ${ }^{4}$
}

Published in: Medical Hypotheses 2008; 70: 384-387

${ }^{1}$ Department of General Practice, School for Public Health and Primary Care (CAPHRI), Maastricht University Medical Centre, Maastricht, the Netherlands. ${ }^{2}$ Department of Clinical Psychological Science, Maastricht University, Maastricht, the Netherlands. ${ }^{3}$ Department of Health Ethics and Philosophy, School for Public Health and Primary Care (CAPHRI), Maastricht University, Maastricht, the Netherlands. ${ }^{4}$ Department of Respiratory Medicine, Maastricht University Medical Centre, Maastricht, the Netherlands. 


\section{ABSTRACT}

Chronic obstructive pulmonary disease (COPD) is a major public health problem. The use of spirometry for early detection of COPD is a current issue of debate because of a lack of convincing evidence for the additive positive effect of spirometry on smoking cessation. In this article, we present conditions under which early detection of COPD and confrontation may be effective, highlighting the principles of "confrontational counselling". Confronting patients with COPD is not an isolated approach but should be integrated into state-of-the-art smoking cessation treatment. Confrontational counselling should consist of several counselling sessions on an individual, face-to-face level, under supervision of a trained smoking cessation specialist, and in combination with evidence-based pharmacological treatment for smoking cessation. 


\section{BACKGROUND}

Chronic obstructive pulmonary disease (COPD) is one of the leading causes of death worldwide. ${ }^{1-3}$ An issue of debate is the use of spirometry for early detection of COPD because convincing evidence for the additive positive effect of spirometry on smoking cessation is lacking. ${ }^{4-10}$ Results from clinical investigations will have to show whether spirometry for smoking cessation is a medically effective intervention, and in how far patients perceive it as ethically acceptable. In this article, we will present five conditions under which early detection of COPD and confrontation may be effective, highlighting the principles of "confrontational counselling".

\section{PRINCIPLES OF CONFRONTATIONAL COUNSELLING}

1. Integrated treatment. Spirometry is not the end but the beginning of treatment and should therefore be integrated into state-of-the-art smoking cessation interventions. It is not effective, or maybe even counter-productive, to merely perform spirometry in patients without offering any established follow-up smoking cessation treatment. Confronting patients with a serious disease that has a bad prognosis might arouse fear. Fear arousal should not be a goal in itself and it is not likely to automatically lead to the desired action (smoking cessation). It can only be effective if one can make the patient understand that there is an effective and feasible therapy for the disease, i.e. quit smoking, and that there is a real chance for the patient to succeed by making use of the offered smoking cessation treatment.

2. Counselling. Confronting smokers with the results of spirometry for smoking cessation should be part of an intensive counselling programme. As stated earlier, smokers try to reduce cognitive dissonance by playing down their own perceived risk of disease, or by denying or avoiding information about the dangers of smoking. ${ }^{11-14}$ Altering such irrational cognitions is time-consuming which makes this approach unsuitable for brief advice. Confrontational counselling preferably consists of several consultations (e.g., four to six sessions of three quarters of an hour) spread over several weeks. This offers the patient the possibility to process the new information and to reflect upon his or her smoking behaviour.

3. Individual approach. Confrontational counselling for smoking cessation should be performed on an individual, face-to-face level. The most crucial part of the counselling is to confront the smoker with his or her personal susceptibility to the hazards of smoking: the own diagnosis of COPD, leaving no room for the smoker to deny or avoid this information or to hide behind irrational beliefs. Group counselling for smoking cessation is known to be effective in general terms ${ }^{15}$, but is probably not the best design when it comes to confrontational counselling. During the individual 
counselling session, the patient should be encouraged to put all thoughts, beliefs, and behaviours towards smoking - all of them are highly personal - on the table. The patient should be stimulated to articulate irrational cognitions and to give motivational statements.

4. Pharmacotherapy. Confrontational counselling should be combined with the use of effective pharmacotherapy. The combination of pharmacological treatment with behavioural support is superior to either alone. ${ }^{15-18}$ As smoking cessation is the cornerstone of treatment in COPD, patients should be offered the best available evidence-based treatment for smoking cessation. This is particularly necessary in patients who have just learned that they have an irreversible disease with a prognosis which worsens in case of continued smoking. The psychological consequences of a failed quit attempt in these patients should not be underestimated.

5. Smoking cessation specialists. Confrontational counselling should be provided by (easily accessible) smoking cessation specialists who have the necessary knowledge and skills. Physicians generally agree that smoking cessation treatment is part of their job. However, many of them perceive lack of time, knowledge and skills as important barriers towards discussing smoking cessation. ${ }^{19}$ Confronting smokers with previously undetected COPD as part of smoking cessation treatment requires special knowledge and skills which mostly derive from the principles of cognitive behavioural therapy. ${ }^{20}$ Therefore, confrontational counselling should be left to health-care providers who are specially trained and who have sufficient time for smoking cessation treatment. A designated group of health-care providers may be (pulmonary) nurses.

\section{TESTING THE EFFICACY OF CONFRONTATIONAL COUNSELLING}

We hypothesise that early detection of COPD and spirometry for smoking cessation is a potentially effective approach using the principles of confrontational counselling. We are currently conducting a randomized controlled trial to test this hypothesis (ISRCTN 64481813): we compare two interventions of exactly the same counselling intensity combined with an antidepressant for smoking cessation (Notrilen ${ }^{\circledR}$ ) in smokers with previously undetected mild to moderate irreversible airflow obstruction. The experimental group receives confrontational counselling, the control group is treated as "healthy smokers", based on a national guideline for health education and promotion. 


\section{REFERENCES}

1 Global Initiative for Chronic Obstructive Lung Disease. Global strategy for the diagnosis, management, and prevention of chronic pulmonary disease. Executive summary: NHLBI / WHO, 2005

2 World Health Organisation (WHO). The World Health Report. Reducing risks, promoting healthy life. Geneva: WHO, 2002

3 Murray CJ, Lopez AD. Alternative projections of mortality and disability by cause 1990-2020: Global Burden of Disease Study. Lancet 1997; 349:1498-1504

4 Boushey H, Enright P, Samet J. Spirometry for Chronic Obstructive Pulmonary Disease Case Finding in Primary Care? Am J Respir Crit Care Med 2005; 172:1481-1482

5 Enright PL, Studnicka M, Zielinski J. Spirometry to detect and manage chronic obstructive pulmonary disease and asthma in the primary care setting. Eur Respir Mon 2005; 31:1-14

6 White P. Should we use spirometry in the early detection of COPD? Eur Respir J 2005; 26:558-559

7 Gorecka D, Bednarek M, Nowinski A, et al. Diagnosis of airflow limitation combined with smoking cessation advice increases stop-smoking rate. Chest 2003; 123:1916-1923

8 Wilt TJ, Niewoehner D, Kim C-B, et al. Use of Spirometry for case finding, diagnosis, and management of chronic obstructive pulmonary disease (COPD). Evidence Report/Technology Assessment No. 121 (Prepared by the Minnesota Evidence-based Practice Center under Contract No. 290-02-0009.) AHRQ Publication No. 05-E017-2. Rockville, MD.: Agency for Healthcare Research and Quality, 2005

9 Enright P. Does screening for COPD by primary care physicians have the potential to cause more harm than good? Chest 2006; 129:833-835

10 Wilt TJ, Niewoehner D, Kane RL, et al. Spirometry as a motivational tool to improve smoking cessation rates: a systematic review of the literature. Nicotine Tob Res 2007; 9:21-32

11 Chapman S, Wong WL, Smith W. Self-exempting beliefs about smoking and health: differences between smokers and ex-smokers. Am J Public Health 1993; 83

12 Halpern MT. Effect of smoking characteristics on cognitive dissonance in current and former smokers. Addict Behav 1994; 19:209-217

13 Lee C. Perceptions of immunity to disease in adult smokers. Journal of Behavioral Medicine 1997; 12:267-277

14 McMaster C, Lee C. Cognitive dissonance in tobacco smokers. Addict Behav 1991; 16:349-353

15 Stead LF, Lancaster T. Group behaviour therapy programmes for smoking cessation. Cochrane Database Syst Rev 2006

16 Silagy C, Lancaster T, Stead LF, et al. Nicotine replacement therapy for smoking cessation. Cochrane Database Syst Rev 2004

17 Hughes JR, Stead LF, Lancaster T. Antidepressants for smoking cessation. Cochrane Database Syst Rev 2004

18 Lancaster T, Stead LF. Individual behavioural counselling for smoking cessation. Cochrane Database Syst Rev 2004

19 Vogt F, Hall S, Marteau TM. General practitioners' and family physicians' negative beliefs and attitudes towards discussing smoking cessation with patients: a systematic review. Addiction 2005; 100:1423-1431 20 Beck AT. Thinking and depression: theory and therapy. Arch Gen Psychiatry 1964; 10:561-571 



\title{
Chapter 5
}

\section{Efficacy of confrontational counselling for smoking cessation in smokers with previously undiagnosed mild to moderate airflow limitation: study protocol of a randomized controlled trial}

\author{
Daniel Kotz ${ }^{1}$ \\ Geertjan Wesseling ${ }^{2}$ \\ Marcus J.H. Huibers ${ }^{3}$ \\ Onno C.P. van Schayck ${ }^{1}$
}

Published in: BMC Public Health 2007; 7: 332

${ }^{1}$ Department of General Practice, School for Public Health and Primary Care (CAPHRI), Maastricht University Medical Centre, Maastricht, the Netherlands. ${ }^{2}$ Department of Respiratory Medicine, Maastricht University Medical Centre, Maastricht, the Netherlands. ${ }^{3}$ Department of Clinical Psychological Science, Maastricht University, Maastricht, the Netherlands 


\section{ABSTRACT}

\section{Background}

The use of spirometry for early detection of chronic obstructive pulmonary disease (COPD) is still an issue of debate, particularly because of a lack of convincing evidence that spirometry has an added positive effect on smoking cessation. We hypothesise that early detection of COPD and confrontation with spirometry for smoking cessation may be effective when applying an approach we have termed "confrontational counselling"; a patient-centred approach which involves specific communication skills and elements of cognitive therapy. An important aspect is to confront the smoker with his/her airflow limitation during the counselling sessions. The primary objective of this study is to test the efficacy of confrontational counselling in comparison to regular health education and promotion for smoking cessation delivered by specialized respiratory nurses in current smokers with previously undiagnosed mild to moderate airflow limitation.

\section{Methods/Design}

The study design is a randomized controlled trial comparing confrontational counselling delivered by a respiratory nurse combined with nortriptyline for smoking cessation (experimental group), health education and promotion delivered by a respiratory nurse combined with nortriptyline for smoking cessation (control group 1), and "care as usual" delivered by the GP (control group 2). Early detection of smokers with mild to moderate airflow limitation is achieved by means of a telephone interview in combination with spirometry. Due to a comparable baseline risk of airflow limitation and motivation to quit smoking, and because of the standardization of number, duration, and scheduling of counselling sessions between the experimental group and control group 1, the study enables to assess the "net" effect of confrontational counselling. The study has been ethically approved and registered.

\section{Discussion}

Ethical as well as methodological considerations of the study are discussed in this protocol. A significant and relevant effect of confrontational counselling would provide an argument in favour of early detection of current smokers with airflow limitation. Successful treatment of tobacco dependence in respiratory patients requires repeated intensive interventions. The results of this study may also show that respiratory nurses are able to deliver this treatment and that intensive smoking cessation counselling is more feasible for respiratory nurses than for physicians who often lack time. 


\section{BACKGROUND}

Chronic obstructive pulmonary disease (COPD) is a preventable and treatable disease which is characterized by airflow limitation that is not fully reversible. ${ }^{1}$ Spirometry is the gold standard for the diagnosis and assessment of the disease. ${ }^{1}$ COPD is currently the fifth leading cause of death worldwide ${ }^{2}$, and projections for 2020 indicate further increase in global mortality, placing COPD on the third position of lethal diseases. ${ }^{3}$ Cigarette smoking is by far the most important risk factor for COPD, and smoking cessation is the single most effective way to reduce the risk of developing COPD and to affect the outcome in patients at all stages of the disease., ${ }^{4,5}$

Underdiagnosis of COPD is a worldwide problem. ${ }^{6}$ Most patients present to their doctor for various other reasons but often have respiratory symptoms, and in those who do present with respiratory symptoms, COPD is not always suspected nor diagnosed. ${ }^{7}$ Because of the irreversible and progressive nature of the disease, early intervention is important. However, the use of spirometry for early detection of COPD is still an issue of debate. ${ }^{8-10}$ The most important counterargument is that there is no convincing evidence that spirometry has an added positive effect on smoking cessation. ${ }^{11-13}$

\section{Why use spirometry for smoking cessation?}

In theory, spirometry might be useful as a motivational tool for smoking cessation in smokers who are at risk of developing (or have) COPD. While most smokers acknowledge that smoking is dangerous, many trivialize their own perceived risk of the disease, or deny or avoid information about the dangers of smoking in order to reduce cognitive dissonance. ${ }^{14-18}$ One might therefore expect that confronting smokers with an objectively (by spirometry) identified negative consequence of smoking (airflow limitation) positively affects the outcome of their quit attempt. This idea was already proposed in the 1960's by Peters and Ferris who argued that assessing the negative effects of smoking on lung function "might serve as a lever to influence the young adult to reduce his smoking habits". ${ }^{19}$ Since then, various studies have been performed to study the efficacy of spirometry for smoking cessation. The results, however, are inconclusive as shown in the systematic review by Wilt et al. on spirometry as a motivational tool for smoking cessation. ${ }^{11,12}$ Also, previous studies have one or more important methodological limitations such as unstandardized counselling intensity, incomparable or uncontrolled use of pharmacological aids for smoking cessation between experimental and control group, and different (or unclear) baseline levels of lung function and motivation to quit smoking. More well-designed research is needed to assess the efficacy of spirometry for smoking cessation. 


\section{Hypothesis and research questions}

We hypothesise that early detection of COPD and confrontation with spirometry for smoking cessation may be effective if the following approach we have termed "confrontational counselling" is applied. ${ }^{20}$ Confronting patients with COPD is not an isolated approach but should be integrated into state-of-the-art smoking cessation treatment. Confrontational counselling should consist of several counselling sessions on an individual, face-to-face level, under supervision of a trained smoking cessation specialist, and in combination with evidence-based pharmacological treatment for smoking cessation.

Our primary research question is: what is the efficacy of confrontational counselling in comparison to regular health education and promotion for smoking cessation delivered by specialized respiratory nurses in current smokers with previously undiagnosed mild to moderate airflow limitation (i.e. GOLD ${ }^{1}$ stage 1 and 2 COPD) with regard to prolonged abstinence from smoking during a period of 12 months?

In this group of smokers with previously undiagnosed mild to moderate airflow limitation we want to address the following secondary research questions:

1. Which baseline characteristics are predictors of outcome (i.e. 12-month prolonged abstinence from smoking)?

2. What are the effects of early detection of airflow limitation and smoking cessation on lung function, perceived specific health-related complaints, quality of life, and mental health after 12 months follow-up?

3. What is the cost-effectiveness and cost-utility of early detection in combination with confrontational counselling delivered by respiratory nurses?

4. What are the effects of labelling of disease (COPD) on self-efficacy, perceived health status, quality of life and mental health?

5. What are the ethical considerations of early detection of airflow limitation and subsequent confrontational counselling for smoking cessation?

\section{METHODS/DESIGN}

In short, the design of this study is a randomized controlled trial comparing confrontational counselling delivered by a respiratory nurse (RN) combined with nortriptyline for smoking cessation (experimental group), health education and promotion delivered by a RN combined with nortriptyline for smoking cessation (control group 1), and "care as usual" delivered by the GP (control group 2). Early detection of smokers with mild to moderate airflow limitation is achieved by means of a telephone interview in combination with spirometry. For an overview of the study design see figure 1. 
The efficacy of smoking cessation interventions in clinical studies depends on the characteristics of the study population, the intensity of behavioural support, and the use of pharmacological aids for smoking cessation. ${ }^{21}$ In order to isolate the effect of confrontational counselling on smoking cessation, other factors that are associated with the outcome must be standardized between the comparison groups. All participants of this trial have previously undetected mild to moderate airflow limitation. Participants from both the experimental group and control group 1 receive an equally intensive counselling (in terms of number, duration, and scheduling of counselling visits) and dosage of nortriptyline for smoking cessation. Another reason for using an active control is that it would not be ethically sound to withhold smokers with airflow limitation from smoking cessation treatment.

\section{Sample size calculation}

The primary research question aims at a contrast in efficacy between the experimental group (confrontational counselling) and control group 1 (health education and promotion). Therefore, the calculation of the sample size is based on the identification of a difference in proportion of prolonged abstinence after 12 months between these two groups. We estimated the relevant difference in proportion to be $15 \%$ : $35 \%$ quitters in the experimental group versus $20 \%$ quitters in control group 1. When putting the risk of a type I-error at $5 \%$ and the risk of a type II-error at $20 \%$, 136 participants per group are needed at onset to detect a difference in proportions of $15 \%$. Considering $10 \%$ lost to follow-up, 150 participants per group are needed in the experimental group and control group $1\left(136 \times 0.9^{-1}\right)$. We expect a larger difference between the experimental group (35\%) and control group $2\left(8 \%{ }^{22}\right)$. Therefore, less participants are needed in control group 2 . We used a formula for the calculation of sample sizes of unequal groups and set the ratio between the experimental group and control group 2 at 3:1. This resulted in a minimum of 32 participants in control group 2 (taking into account $10 \%$ lost to follow-up).

\section{Preparation of the trial}

In preparation of the trial, all GPs in Dutch and Belgian Limburg (the area surrounding the city of Maastricht) have been informed about the study. We have prepared an office for the screening, counselling, and follow-up visits of participants at Medical Centre Annadal, Maastricht. We have built a relational database for the control of all study events and the collection of data. This is very important because of the complexity of the study.

\section{Recruitment of participants}

Subjects are recruited in the general population (through advertisements in local newspaper, flyers, posters, and mailings to households) and in primary care practices (during consultations and through posters and personalized mailings) in Dutch and Belgian Limburg. The text in the advertisements, on flyers, and on posters explains 
that Maastricht University is performing a study on smoking cessation treatment in which individual counselling is combined with medication for smoking cessation. Current smokers aged 35 to 70 years, who are motivated to quit smoking, are asked to contact us by telephone or by e-mail. We also refer to a website with information about this study. No information about the target condition we are looking for (airflow limitation) is given to participants during recruitment.

\section{In- and exclusion criteria and process of eligibility screening}

Eligibility is screened in two steps during an initial telephone interview followed by spirometry. The following inclusion criteria are checked during the telephone interview: smoking history of 10 or more pack years*, being motivated to stop smoking, being competent to read and speak Dutch, and reporting a respiratory symptom, defined as an affirmative answer to at least one of the following three questions: "Do you cough regularly?", "Do you cough up phlegm (sputum) when you don't have a cold?" or "Have you been shorter of breath lately?". Exclusion criteria are: evidence of a prior respiratory diagnosis, defined by an affirmative answer to the question "Do you have COPD, chronic bronchitis, asthma or asthmatic bronchitis?". Participants are also not allowed to have undergone a lung function test (spirometry) during the preceding 12 months. One or more contraindications for using the smoking cessation medication (nortriptyline) are also reasons for exclusion, among others the current use of anti-depressants. Nortriptyline is a tricyclic anti-depressant which should not be used for smoking cessation in conjunction with another anti-depressant. After the end of the telephone interview, an appointment for spirometry at Medical Centre Annadal is scheduled. Subjects are eligible for participation who have airflow limitation defined as post-bronchodilator (post-bd.) Forced Expiratory Volume in one second (FEV1) / Forced Vital Capacity (FVC) $<70 \%$ in combination with post-bd. FEV $1>50 \%$ of predicted value; i.e. mild (GOLD 1) or moderate (GOLD 2) airflow limitation, according to the international GOLD guideline1. The results of spirometry are not discussed during or directly after spirometry.

Subjects with severe airflow limitation (post-bd. $\mathrm{FEV}_{1} / \mathrm{FVC}<70 \%$ in combination with post-bd. $\mathrm{FEV}_{1}<50 \%$ of predicted value) are excluded from participation and advised to contact their GP or a lung physician for further evaluation. Subjects without airflow obstruction (post-bd. $\mathrm{FEV}_{1} / \mathrm{FVC} \geq 70 \%$ ) are also excluded. These smokers are told that despite their normal lung function, they still are at risk of getting other smoking related diseases which are not measured by spirometry, such as cancer or cardiovascular disease. They are strongly recommended to give up smoking. Both groups of excluded smokers get the advice to stop smoking and receive a box with information material about all existing therapies for smoking cessation from the Dutch foundation for a smoke free future (STIVORO).

\footnotetext{
${ }^{*}$ Pack year $=($ cigarettes smoked per day $) \times($ years smoking $) / 20$
} 


\section{Spirometry}

Spirometry is performed by two qualified research assistants under permanent supervision of a pulmonologist (GW) according to the criteria of the American Thoracic Society (ATS) / European Respiratory Society (ERS) task force for standardization of lung function testing ${ }^{23,24}$ using a Vitalograph ${ }^{\circledR} 2120$ (Vitalograph Ltd, Buckingham, England). After a minimum of three acceptable and reproducible FVC manoeuvres, a bronchodilator (500 $\mathrm{\mu g}$ terbutaline) is administered to the subject in preparation for the reversibility test. After 15 minutes, another series of three FVC manoeuvres is performed. All spirometric test results are independently validated by a pulmonologist (GW) and by a specialised lung function laboratory assistant who was not involved in the trial. In case of initial disagreement, consensus is obtained during re-examination.

\section{Informed consent procedure}

Written information about the study is sent to the candidate participant after the telephone interview, along with the informed consent form. The candidate has at least one week time for reflection before spirometry and can contact the researcher or the research assistant (RA) for further information at any time. The informed consent form is signed by the participant in presence of the RA during the visit for baseline spirometry.

The participant information letter gives information about the existence of two intervention groups only. It says that in one group participants receive "care as usual" by their own GP (this is control group 2) and in the other group participants receive treatment from a trained $\mathrm{RN}$. The latter group is in fact a combination of the experimental group (confrontational counselling by RN + nortriptyline) and control group 1 (health education and promotion by RN + nortriptyline). These two groups are identical with regard to the number and duration of counselling sessions and the use of nortriptyline, but differ only concerning the content and style of the behavioural support: confrontational counselling versus health education and promotion. Just this difference in content must not explicitly be mentioned in the participant information letter in order to safeguard the internal validity of the study. We would jeopardize the idea behind early detection of patients with airflow obstruction by means of spirometry if we would speak about "confrontational counselling" or mention the target condition (airflow limitation). Participants must not know that we use results from spirometry as part of one intervention. The design we use is adapted from Zelen's design ${ }^{25,26}$ which may be particularly useful when evaluating the full unbiased impact of screening interventions. ${ }^{27}$

At the end of the study, after the 12-month follow-up visit, all participants will indeed be fully informed about the real nature of the study. All participants and their corresponding GPs will be informed about the result of the spirometry. If a GP needs the 
results of spirometry for the regular care of his/her patient before the end of the study, the required information will be provided. This procedure is approved by the medical ethics committee of Maastricht University and Maastricht University Hospital.

\section{Randomization and planning procedure}

All eligible subjects with previously undetected mild to moderate airflow limitation are contacted by telephone a few days after baseline spirometry to be randomised to one of the three intervention groups (apart from those candidates who changed their mind and who are no longer willing to participate in the study). Also at this moment, the results of spirometry are not discussed. The database of the trial incorporates a randomization system of seven participants per block, allowing an unequal group allocation of $3: 3: 1$; experimental group : control group 1 : control group 2 . When eligible subjects are contacted by telephone, the RA randomises the subject by pressing a button on the computer screen. The database then randomly allocates the subject. Neither the primary researcher nor any other person involved in the study can predict or influence which treatment group the next participant will be allocated to.

After randomization, all treatment and follow-up visits are planned for the whole study period. A schedule with all visits is sent to the participant. At the same time, the GP of each participant is sent a letter informing the GP that the participant is taking part in the study.

\section{Experimental group and control group 1: L-MIS as the common basis of counselling}

Participants from both the experimental group and control group 1 receive counselling delivered by a RN combined with nortriptyline for smoking cessation. The common basis for the counselling is the so-called "L-MIS" protocol for the treatment of nicotine and tobacco addiction which has been implemented among all respiratory nurses in the Netherlands in recent years. ${ }^{28}$

The number of counselling sessions, their duration and scheduling is fixed in both the experimental group and control group 1 (see also figure 1). The first face-to-face counselling session (FC1; day 1, duration 40 minutes) starts with getting acquainted with each other. The RN tries to build up a relationship with the participant which is based on trust. The participant's smoking characteristics are defined by asking about smoking status, cigarettes smoked per day, and readiness to quit. Nicotine addiction is assessed by number of cigarettes per day and moment of smoking the first cigarette after waking up in the morning. The motivation for quitting smoking is assessed and increased by asking the readiness for quitting and reasons for smoking and quitting. Also, the health risks of smoking are discussed and the pros of quitting. The use of nortriptyline for smoking cessation is discussed, including mechanism, dosage, 
administration, and possible side effects (for more information about the study medication see paragraph "Use of nortriptyline for smoking cessation"). The participant starts with the intake of the study medication the same day.

At the beginning of the second face-to-face counselling session (FC2; day 8, duration 40 minutes), the use of the study medication is evaluated and possible side effects are discussed. Barriers of quitting and the most important problems with previous quit attempts are discussed. The RN tries to increase the participant's self-efficacy towards smoking cessation. The focus of the second session is to prepare the participant for the target quit date (TQD). The RN discusses with the participant how to deal with the most important barriers of quitting smoking. The RN anticipates problems with withdrawal, difficult moments, and craving. The RN provides pointers for the TQD and schedules the telephone counselling on that day.

On the TQD (day 14), the participant is counselled on the telephone (TC; duration 5 - 15 minutes). The RN evaluates the quit attempt, discusses difficult moments, and gives advice for quitting and abstaining from smoking.

The third face-to-face counselling session is scheduled directly after the TQD (FC3; day 15, duration 40 minutes) and starts with an evaluation of the quit attempt and the use of the study medication (including possible side effects). The RN discusses with the participants what is going well and what is problematic with this quit attempt. The RN identifies difficult moments and strategies to deal with theses situations in the future. Participants who have not quit smoking yet or who already relapsed are asked about their reasons and are encouraged to try again.

The fourth face-to-face counselling session (FC4; day 22, duration 40 minutes) basically resembles the third session; the RN evaluates the quit attempt and the use of the study medication. As this is the last counselling session, the focus lies on preparing the participant for continuation of the quit attempt during the follow-up period. The participant is asked to continue with the intake of nortriptyline according to the protocol. At the end of the session, the RN asks the participant's feedback on the perceived effectiveness of the behavioural support and the study medication.

All RNs have had initial training in the use of the L-MIS method and are experienced in the treatment of nicotine addiction. All RNs are trained to use a tailored version of the L-MIS protocol which is specifically designed for this trial. The compliance of RNs with the treatment of participants is stimulated by the use of a protocolized treatment manual, including intervention registration forms providing information per session about all the aspects of smoking cessation counselling to be addressed. The RNs are trained to fill out these intervention forms during each counselling session. 


\section{Discriminative component in the experimental group: confrontational counselling}

The number of counselling sessions, the duration, and the scheduling are identical between the experimental group and control group 1. However, specific aspects of "confrontational counselling" are added to the L-MIS in the experimental group which discriminate the treatment from the treatment in control group 1. These aspects derive from the principles of "confrontational counselling" which we have described in more detail elsewhere. ${ }^{20}$ Confronting patients with COPD is not an isolated approach but should be integrated into state-of-the-art smoking cessation treatment. Confrontational counselling should consist of several counselling sessions on an individual, face-to-face level, under supervision of a trained smoking cessation specialist, and in combination with evidence-based pharmacological treatment for smoking cessation.

A key element of confrontational counselling is to confront the participant with his/her airflow limitation during the first counselling session (FC1). The RN discusses the results of the participant's individual baseline spirometry and explains the manifestations of COPD; COPD is a slowly progressive, irreversible but treatable disease. Most importantly in this respect is to make the participant understand that his/her cigarette smoking is the primary cause of the disease. The negative effect of smoking on the lungs is illustrated by showing and comparing images of a normal lung and a "smoker's lung". The participant is asked to recognize common symptoms, functional limitations, and participation problems associated with COPD. The natural history of COPD is discussed and illustrated using the so-called "Fletcher curve" (see also figure 2). ${ }^{29}$ Confronting patients with a serious disease that has a bad prognosis arouses fear. Fear arousal is not a goal in itself and it is not likely to automatically lead to the desired action (smoking cessation). Therefore, when discussing the results of spirometry, the RN tries to make the participant understand that there is an effective and feasible therapy for the disease: smoking cessation. Because airflow limitation has been detected early in the participant, early treatment is possible to avoid further damage. Smoking cessation is the only therapy to reduce the progression of the disease resulting in prolonged life expectancy and improved quality of life. The motivation of the participant to stop smoking in combination with the behavioural counselling offered by the RN and the smoking cessation medication (nortriptyline) increases the chance of the participant to quit smoking and to subsequently improve health. At the end of the session, the participant receives a folder with background information on COPD which is developed by the Dutch College of General Practitioners (NHG). In contrast to the experimental group, participants from control group 1 are not being confronted with the detected airflow limitation. The RN from control group 1 is instructed not to discuss the result of spirometry at any time, but to treat the participant as a "healthy smoker". 
The information about airflow limitation and COPD during the first counselling session probably has impact on the participant. At the beginning of the second counselling session (FC2), the RN asks the participant to reflect on this information. The RN assesses whether the participant has processed the information correctly and provides feedback on the thoughts, feelings, and beliefs the participant has reported. Again, the positive effect of smoking cessation on the history of lung function is stressed and illustrated using the Fletcher curve. This is repeated during later counselling sessions if necessary.

Confrontational counselling comprises more than merely confronting the participant with his/her results from spirometry. It is a patient-centred approach which involves specific communication skills and elements of cognitive therapy. An important condition is a relationship (also known as alliance) between RN and participant in which both roles are equivalent (rather than an expert-recipient relationship). The RN respects the participant's freedom of choice regarding his/her own smoking behaviour. The RN stimulates the participant to reflect on his/her smoking behaviour by carefully listening to the participant's story, using open ended questions, paraphrases, and reflections. Confrontational counselling aims to identify certain cognitions about smoking such as health concerns, risk perception and self-exempting beliefs. The RN tries to challenge irrational beliefs about smoking by raising the smokers consciousness about these beliefs, testing their reality, and by exploring the relationship between beliefs and behaviour. An example for a typical self-exempting belief of a smoker would be: "Smoking is possibly not very harmful because many smokers live long. My grandfather is 85 and he has smoked all his life". The RN will try to challenge this belief for instance by conduction an objective risk assessment, or by exploring biases in the belief itself.

At the end of the first, second, and third counselling session, the RN hands out a smoking cessation diary to the participant. Homework is an essential element in cognitive therapy, and self-monitoring diaries are used as extra input for the counselling sessions. In the first diary (evaluated during FC2), participants have to record their smoking behaviour; they have to count the number of cigarettes smoked and have to describe one situation in which they experience great desire to smoke. In the second diary, participants have to record their thoughts when smoking. Again they have to count the number of cigarettes and have to describe one situation, but also what was on their mind directly before and after lighting up. In the third diary (evaluated during FC3), participants have to describe both behaviour and thoughts in situation when they have great desire to smoke.

To ensure the building of an alliance between RN and participant, it is important that the participant is counselled by one and the same pulmonary nurse during the whole intervention period to prevent contamination between the groups. Consequently, 
each treatment group has its own RNs. All RNs have had initial training in the use of the L-MIS method and are experienced in the treatment of nicotine addiction. Additionally, RNs from the intervention group will receive a four-hour group training in confrontational counselling. The group training is led by a cognitive therapist $(\mathrm{MJH})$ who acts as supervisor throughout the study, and incorporates practical training with a simulation patient. Supervision meetings between RNs and the supervisor will be planned every 6 weeks during the whole intervention period of the trial.

RNs from control group 1 do not receive an introductory group training but receive feedback during regular evaluation meetings between the $\mathrm{RN}$ and the principal investigator.

\section{Control group 2: care as usual by GP}

Participants from control group 2 are referred to their own GP for smoking cessation treatment. They are asked to make an appointment with their GP within the next ten days. They are provided with a referral letter explaining to the GP that the person is participating in a study on smoking cessation. This letter does not give any information about the results from spirometry and the fact that the participant has airflow limitation. The GP is asked to provide the care he/she usually provides to patients who want to quit smoking. In the Netherlands, primary "care as usual" for smoking cessation involves the use of a protocol for low intensity health education and promotion, the so-called "H-MIS". ${ }^{22}$ According to the protocol of the H-MIS, the GP and/or the assistant takes the following steps to assist the smoker during a quit attempt: determine the smoking profile of the smoker, determine the motivation to stop smoking (and increase the motivation if necessary), talk about the barriers of quitting smoking, set a target quit date, discuss the use of smoking cessation aids, and arrange follow-up. A semi-structured interview will be used among participants from control group 2 during the first follow-up visit (day 50) in order to assess whether participants have consulted their GP and which treatment for smoking cessation the GP has delivered.

Neither participants from control group 2 nor their GP will be informed about the results from spirometry and the detected airflow limitation. However, if a GP explicitly requests the results from spirometry they will be provided (see further paragraph "ethical considerations").

\section{Use of nortriptyline for smoking cessation}

Previous research has shown that the combination of counselling and pharmacotherapy is more effective than either alone ${ }^{30-32}$ and international guidelines recommend the use of pharmacotherapy in all patients trying to make a quit attempt. ${ }^{21,33}$ Participants from both the experimental group and control group 1 receive an equal dosage of nortriptyline (Nortrilen ${ }^{\circledR}$ ) for smoking cessation. Nortriptyline is a tricyclic anti- 
depressant which has been shown to be a cheap and effective alternative for the anti-depressant bupropion (Zyban ${ }^{\mathrm{TM}}$ ). ${ }^{30,34}$ Participants start taking nortriptyline on the day of the first counselling visit (FC1, day 1$)$ in which they receive instructions about the use of nortriptyline by the RN. A run-in period of 10 days until the TQD is needed to achieve steady-state blood levels of nortriptyline. From day 1 through day 3, participants take one pill of $25 \mathrm{mg}$ nortriptyline once a day (preferably after dinner). From day 4 through day 7, participants take $50 \mathrm{mg}$ a day (given as two pills of $25 \mathrm{mg}$ ). As from day 8 through the end of the treatment period (day 49), participants take $75 \mathrm{mg}$ a day (given as three pills of $25 \mathrm{mg}$ ). The RN monitors the correct use of the medication and the occurrence of side-effects during the intervention period. In case of unpleasant or severe side-effects, the dosage will be reduced or the use of the medication will be stopped. At the first follow-up visit (day 50) the RA collects and counts the remaining pills.

\section{Follow-up visits}

Three follow-up visits for all participants are scheduled at day 50 (approximately five weeks after the TQD), day 197 (approximately six months after the TQD), and day 379 (approximately twelve months after the TQD; see also figure 1). The TQD in participants from control group 2 is set at 8 weeks after the day participants were randomized and informed about group allocation. We estimated that this would be sufficient time to schedule a consultation with the GP and to prepare stopping smoking, and that the time lag between day of randomization and TQD would be about the same compared to the experimental group and control group 1 (in the latter groups, we account for a delay between day of randomization and start of treatment). Participants receive a reminder letter including a follow-up questionnaire and a cost-diary seven weeks prior to all three visits. The RA calls every participant one week prior to the visit to confirm the appointment. Minor deviations from the scheduling of follow-up visits will be allowed in order to retain as many participants in the study as possible.

At every follow-up visit, participants hand in their questionnaire and cost-diary. The RA briefly discusses the quit attempt. Urine is collected from every self-reported nonsmoker for the analysis of cotinine levels.

During the final follow-up visit, spirometry is repeated in all participants. All spirometric outputs are carefully evaluated by a pulmonologist (GW) and reported to the participant's GP by letter. All participants are asked to consult their GP for information about their lung function and further treatment.

\section{Data collection}

An overview of all measurements per visit is given in table 1 . The paper-and-pencil questionnaires are filled out at home by the participants and are handed in during 
the visits. Completion of the questionnaires takes about 30 minutes. Data from the questionnaires will be double-entered and checked by blinded assistants from the centre for data and information management of Maastricht University (MEMIC).

Urine is collected from every self-reported non-smoker during each follow-up visit to validate non-smoking. The urine is kept in a $100 \mathrm{~mL}$ plastic cup with a screw cap and temporarily stored in a refrigerator for a maximum of seven days before delivery to the laboratory of the Department of Health Risk Analysis and Toxicology (GRAT) of Maastricht University. The concentration of cotinine in urine is measured by a highly specific radioimmunoassay using monoclonal antibodies. ${ }^{35}$ The reagents for the assay are obtained from the Department of Biochemistry, Brandeis University, Massachusetts, USA.

The analysts assessing the urine cotinine levels and all assistants entering data from questionnaires are kept blind to the group allocation of participants.

\section{Data analysis}

The primary outcome measure is prolonged abstinence from smoking during a period of 12 months after the TQD. Prolonged abstinence is defined as follows: abstinent from smoking at all three follow-up visits; at day 50 (approximately 5 weeks after the TQD), day 197 (after six months), and day 379 (after 12 months). Participants are allowed to miss the second follow-up visit (day 197) if they have been abstinent from smoking at the first (day 50) and the last follow-up visit (day 379; interpolation). A participant is defined as abstinent from smoking at a follow-up visit if both of the following two conditions are met: urine cotinine level $<50 \mathrm{ng} / \mathrm{mL}^{36}$ and self-reported quitter, not having smoked a single cigarette since stopped smoking.

All randomized subjects will be included in the analysis and subjects not showing up at the follow-up visit or with a missing value on one of the two above measures are regarded as smokers (intention-to-treat analysis). Statistical difference in primary outcome will be analysed using Chi-square tests.

The secondary research questions will be analysed as follows (see the last paragraph of the introduction for an overview of all secondary research questions).

1. Baseline predictors of outcome will be analysed by regressing the primary outcome measure (12-month prolonged abstinence) on candidate predictors measured at baseline (such as age, sex, airflow limitation, previous quit attempts, or nicotine dependence) in a multivariate logistic regression model, controlling for treatment group. 
2. The health effects of smoking cessation will be analysed by regressing measures of lung function, perceived specific health-related complaints, quality of life, and mental health on abstinence at each follow-up visit using linear regression models.

3. The cost-effectiveness and cost-utility of early detection of airflow limitation in combination with smoking cessation will be analysed using data from the cost diaries. ${ }^{37}$ Participants fill out these diaries during three periods of six weeks each: during the intervention until the first follow-up visit (day 50), during the period until the second follow-up visit (day 197), and during the period until the last follow-up visit (day 379). The economic evaluation is based on direct medical costs (e.g., treatment costs, spirometry), direct non-medical costs (e.g., reimbursement of travelling expenses), and on indirect costs (e.g., sickness absence), which are related to respiratory complaints. Effects are measured in physical units (such as number of successful quit attempts, FEV 1 ) and Quality Adjusted Life Years (QALY'S) are measured with the EuroQol (EQ-5D). ${ }^{38,39}$

4. The effects of labelling of disease (COPD) will be analysed by mediation and moderation analyses using linear and logistic regression models. The outcome variable in these analyses is abstinence from smoking at the first follow-up visit. Only data from the experimental group and control group 1 will be used.

5. The ethical considerations of early detection of airflow limitation will be analysed in a qualitative analysis using data from the ethical exit interviews which are performed in participants attending the last follow-up visit.

\section{Ethical approval, review, and registration of the trial}

Participants of this study are not fully informed about the real purpose of the study at the beginning, which is to detect and confront smokers with airflow limitation. Participants from control group 1 and control group 2 are not informed about their results of spirometry during the intervention and follow-up period. This approach is necessary to assess the additional effect of early detection of and confrontation with airflow limitation above the effects of individual counselling and medication use. As already explained in the paragraph "informed consent", all participants as well as their GPs will be fully informed about the purpose of the study and the results of spirometry after the last follow-up visit.

This procedure is approved by the medical ethics committee of Maastricht University and Maastricht University Hospital. We believe that it is ethical to withhold information about the results from spirometry to participants of this study for at least two reasons. The first reason is that the smokers participating in this trial would probably not have been diagnosed with airflow limitation outside the trial setting early due to the problem of underdiagnosis of COPD in primary care. The second reason is that all 
smokers from this trial receive the most effective therapy for airflow limitation from ither the RN or their own GP, which is smoking cessation treatment.

Data from the ethical analysis, which is based on the interviews with participants during the last follow-up visit, should provide information about the participants' view on the ethical aspects of this trial.

The protocol of this study was extensively reviewed by the funding organizations; the Netherlands Asthma Foundation (3.4.03.27), PICASSO for COPD, and Maastricht University Hospital. The study is registered at the Netherlands Trial Register (ISRCTN 64481813).

\section{Time frame}

The recruitment, inclusion, randomization, and treatment of participants will start in 2005 and will continue until 2007. The follow-up of participants is planned until the year 2008. All data will be continuously collected, entered, and cleaned. The analysis of data regarding the primary research question will not be initiated before the completion of follow-up and data collection in the last participants (during the course of the year 2008).

\section{DISCUSSION}

We presented the protocol of a study assessing the efficacy of confrontational counselling for smoking cessation in current smokers with not earlier diagnosed mild to moderate airflow limitation (i.e. GOLD stage 1 and 2 COPD). The design of this study is a randomized controlled trial comparing confrontational counselling delivered by a respiratory nurse (RN) combined with nortriptyline for smoking cessation (experimental group), health education and promotion delivered by a RN combined with nortriptyline for smoking cessation (control group 1), and "care as usual" delivered by the GP (control group 2). We hypothesise that early detection of COPD and confrontation with spirometry for smoking cessation is more effective than regular health education and promotion and primary care as usual for smoking cessation.

In the design of this randomized controlled trial, the baseline risk of all participants is the same; they all have previously undetected airflow limitation. Only participants from the experimental group are confronted with their disease. Participants from the two control groups are not informed before the end of the trial. All other factors which are known to be associated with abstinence from smoking are standardised in both the experimental group and control group 1: type of counsellor (RN), type of counselling (face-to-face and by telephone), number and duration of counselling sessions, and type (nortriptyline) and dosage of smoking cessation medication. 
Therefore, we are able to assess the "net" effect of confronting and counselling smokers with COPD.

There are several critical success factors to be mentioned. A large number of smokers will have to be screened in order to obtain enough eligible participants. No smokers with known airflow limitation are allowed to enter the trial. Also, during the whole recruitment period, candidate participants must not be informed about the real purpose of the study at the beginning: to detect and confront smokers with airflow limitation. This means that a large number of smokers generally interested in quitting must be recruited in order to filter out eligible smokers with airflow limitation.

We expect eligible smokers to have a strong preference to be placed in one of the groups receiving counselling by a $\mathrm{RN}$ in combination with smoking cessation medication, because they may find this intervention more effective than care as usual by their own GP. Therefore, we expect a lower compliance in participants from control group 2 and differential lost to follow-up in this group. It should be noted here that the intervention in control group 2 is probably not care as usual for smoking cessation as it appears in primary care. This is because smokers are more or less "referred" from the study team to their GP and are likely to ask for the same smoking cessation medication as participants randomized to the other two groups (nortriptyline).

Blinding of neither participants nor RNs is possible, because we want to assess behavioural interventions. All participants know what kind of counselling they receive, as do the respiratory nurses who provide the counselling. However, analysts assessing the cotinine levels and assistants entering data from questionnaires are blinded for the group allocation of participants.

The primary outcome, 12-month prolonged abstinence from smoking, is estimated from abstinence measures at three time points. This outcome is therefore no perfect measure of continuous outcome, but it is a feasible estimation, which is very usual in this field of research. Abstinence from smoking is the primary endpoint of this study, but can be regarded as a surrogate for the expected long-term positive effects of smoking cessation on health.

\section{CONCLUSIONS}

Early intervention in COPD is of paramount importance because of the irreversible and progressive nature of the disease. The use of spirometry for early detection of COPD still is an issue of debate because of a lack of convincing evidence that spirometry has an added positive effect on smoking cessation. A significant and relevant 
effect of confrontational counselling compared to regular health education and promotion for smoking cessation would provide an argument in favour of early detection of current smokers with airflow limitation. Successful treatment of tobacco dependence in respiratory patients requires repeated intensive interventions. The results of this study may show that RNs are able to deliver this treatment and that intensive smoking cessation counselling is more feasible for RNs than for physicians who often lack time. 


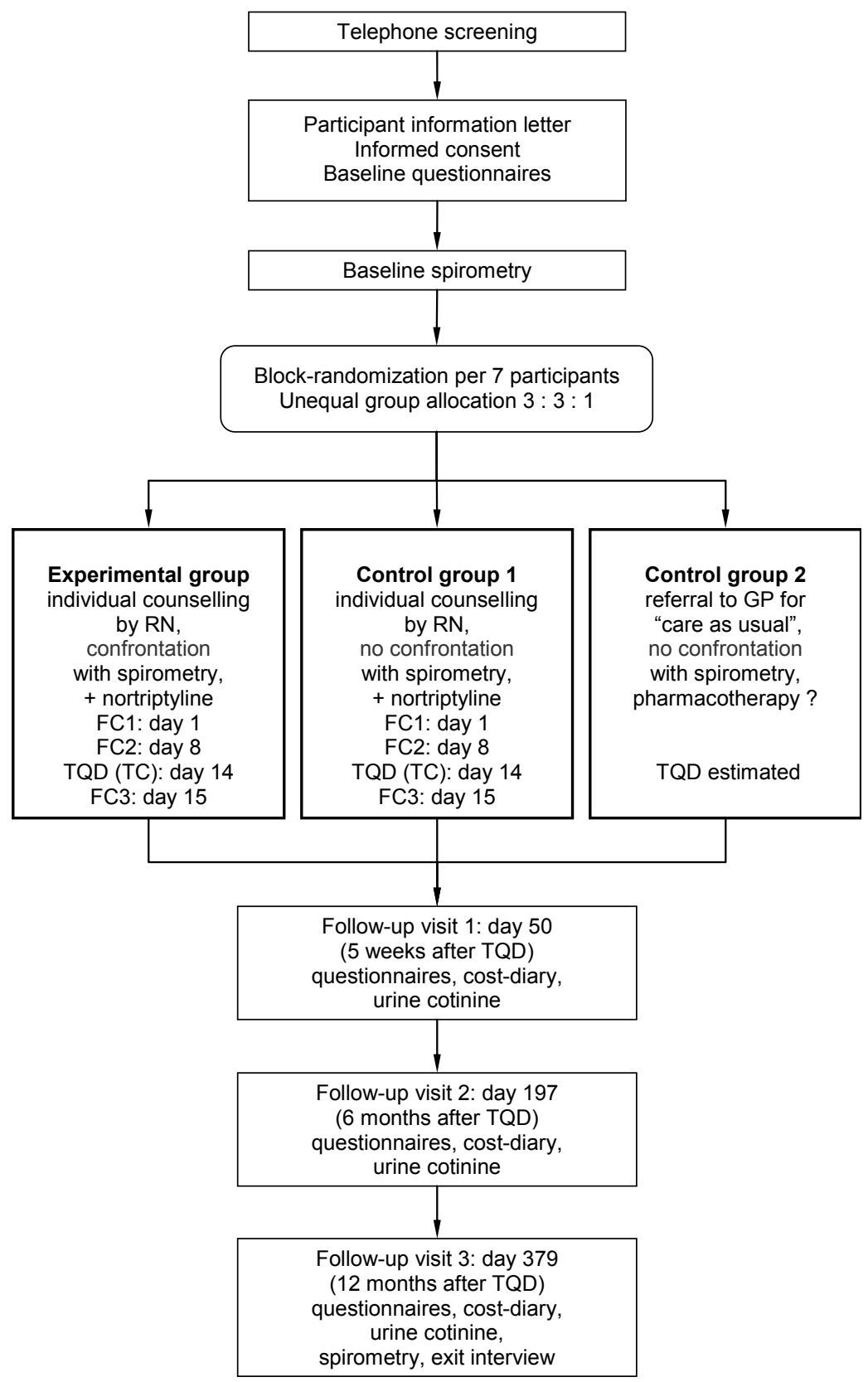

Figure 1: Design. $\mathrm{FC}=$ face-to-face counselling session; $\mathrm{TC}=$ telephone counselling session; $\mathrm{TQD}=$ target quit date; $\mathrm{RN}=$ respiratory nurse; $\mathrm{GP}=$ general practitioner. 


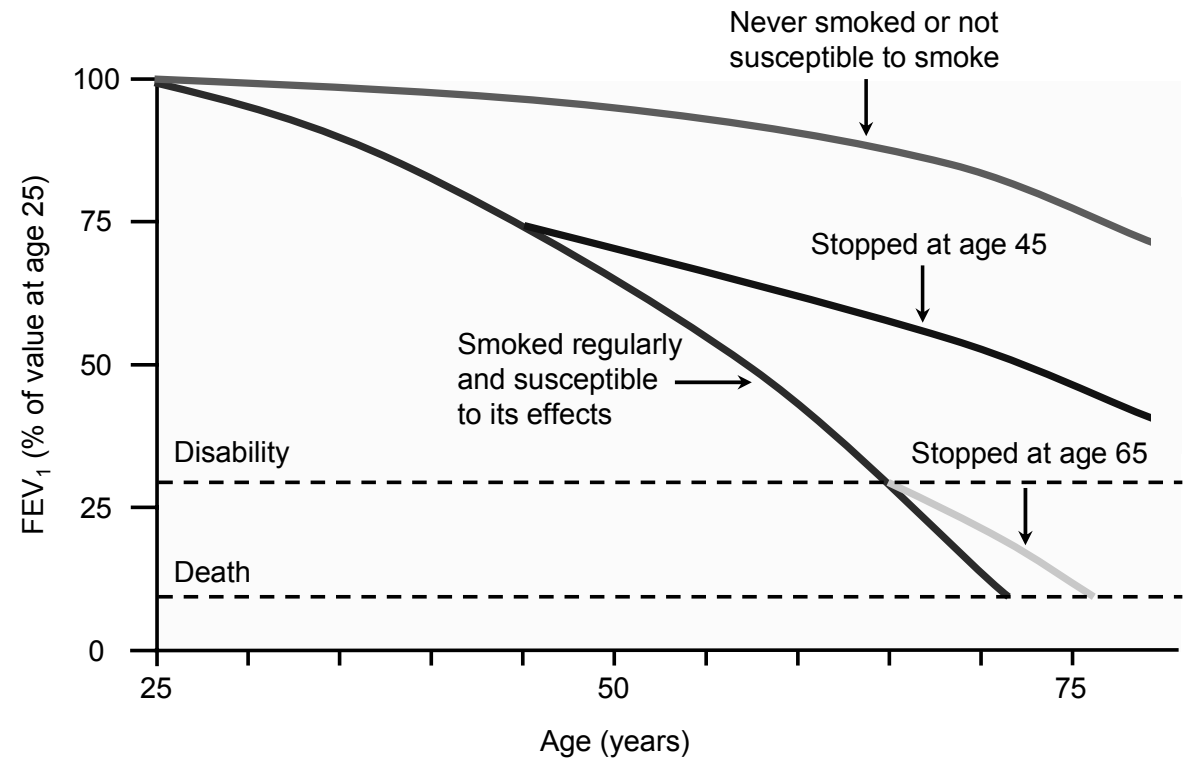

Figure 2: "Fletcher curve". Adapted from Fletcher \& Peto (1977): The natural history of chronic airflow obstruction $^{29}$ 
Table 1: Overview of measurements per visit.

\begin{tabular}{|c|c|c|c|c|}
\hline Measurement & Baseline visit & $\begin{array}{c}\text { Follow-up } \\
\text { visit } 1 \\
\text { (day } 50 \text { ) }\end{array}$ & $\begin{array}{c}\text { Follow-up } \\
\text { visit } 2 \\
\text { (day 197) }\end{array}$ & $\begin{array}{c}\text { Follow-up } \\
\text { visit } 3 \\
\text { (day } 379 \text { ) }\end{array}$ \\
\hline Demographic characteristics & $\bullet$ & & & \\
\hline \multicolumn{5}{|l|}{ Smoking: } \\
\hline Tobacco use and quit attempts ${ }^{40}$ & $\bullet$ & $\bullet$ & $\bullet$ & $\bullet$ \\
\hline Fagerström Test for Nicotine Dependence (FTND) $)^{41}$ & $\bullet$ & & & \\
\hline \multicolumn{5}{|l|}{ Health perception: (self-constructed questions) } \\
\hline health concerns & - & • & - & • \\
\hline risk perception & • & • & • & • \\
\hline self-exempting beliefs & $\bullet$ & $\bullet$ & $\bullet$ & $\bullet$ \\
\hline \multicolumn{5}{|l|}{ Respiratory health complaints: } \\
\hline COPD diagnostic questionnaire ${ }^{42,43}$ & $\bullet$ & & & \\
\hline Medical Research Council (MRC) dyspnoea scale ${ }^{44,45}$ & $\bullet$ & & $\bullet$ & $\bullet$ \\
\hline Clinical COPD Questionnaire (CCQ) ${ }^{46,47}$ & $\bullet$ & • & $\bullet$ & $\bullet$ \\
\hline \multicolumn{5}{|l|}{ Health-related quality of life: } \\
\hline EuroQol (EQ-5D) $)^{38,39}$ & $\bullet$ & $\bullet$ & $\bullet$ & $\bullet$ \\
\hline Short-form 36-item questionnaire (SF-36) $)^{48,49}$ & $\bullet$ & $\bullet$ & $\bullet$ & $\bullet$ \\
\hline $\begin{array}{l}\text { Chronic Respiratory Questionnaire self-reported } \\
\text { (CRQ-SR) }\end{array}$ & $\bullet$ & $\bullet$ & $\bullet$ & $\bullet$ \\
\hline \multicolumn{5}{|l|}{ Mental health: } \\
\hline Beck Depression Inventory $(\mathrm{BDI})^{52}$ & • & $\bullet$ & - & $\bullet$ \\
\hline Hospital Anxiety and Depression Scale (HADS) $)^{53-55}$ & $\bullet$ & $\bullet$ & $\bullet$ & $\bullet$ \\
\hline $\begin{array}{l}\text { Cost diary: measurement of direct en indirect medical and } \\
\text { non-medical costs }{ }^{37}\end{array}$ & & $\bullet$ & $\bullet$ & $\bullet$ \\
\hline \multicolumn{5}{|l|}{ Physical measurements: } \\
\hline Physical height and weight & $\bullet$ & $\bullet$ & $\bullet$ & $\bullet$ \\
\hline Post-bronchodilator spirometry & $\bullet$ & & & $\bullet$ \\
\hline Urine cotinine (only in self- reported quitters) & & • & • & • \\
\hline
\end{tabular}




\section{REFERENCES}

1 Global Initiative for Chronic Obstructive Lung Disease. Global strategy for the diagnosis, management, and prevention of chronic pulmonary disease. Executive summary (http://www.goldcopd.com): NHLBI / WHO, 2006

2 World Health Organisation (WHO). The World Health Report. Reducing risks, promoting healthy life. Geneva: WHO, 2002

3 Murray CJ, Lopez AD. Alternative projections of mortality and disability by cause 1990-2020: Global Burden of Disease Study. Lancet 1997; 349:1498-1504

4 Pauwels RA, Buist AS, Calverley PM, et al. Global strategy for the diagnosis, management, and prevention of chronic obstructive pulmonary disease. NHLBI/WHO Global Initiative for Chronic Obstructive Lung Disease (GOLD) Workshop summary. Am J Respir Crit Care Med 2001; 163:1256-1276

5 Anthonisen NR, Connett JE, Kiley JP, et al. Effects of smoking intervention and the use of an inhaled anticholinergic bronchodilator on the rate of decline of FEV1. The Lung Health Study. J Am Med Assoc 1994; 272:1497-1505

6 Pauwels PRA, Rabe KF. Burden and clinical features of chronic obstructive pulmonary disease (COPD). Lancet 2004; 364:613-620

7 van Schayck CP, van der Heijden FMMA, van den Boom G, et al. Underdiagnosis of asthma: is the doctor or the patient to blame? The DIMCA project. Thorax 2000; 55:562-565

8 Boushey H, Enright P, Samet J. Spirometry for Chronic Obstructive Pulmonary Disease Case Finding in Primary Care? Am J Respir Crit Care Med 2005; 172:1481-1482

9 Enright PL, Studnicka M, Zielinski J. Spirometry to detect and manage chronic obstructive pulmonary disease and asthma in the primary care setting. Eur Respir Mon 2005; 31:1-14

10 White P. Should we use spirometry in the early detection of COPD? Eur Respir J 2005; 26:558-559

11 Wilt TJ, Niewoehner D, Kane RL, et al. Spirometry as a motivational tool to improve smoking cessation rates: a systematic review of the literature. Nicotine Tob Res 2007; 9:21-32

12 Wilt TJ, Niewoehner D, Kim C-B, et al. Use of Spirometry for case finding, diagnosis, and management of chronic obstructive pulmonary disease (COPD). Evidence Report/Technology Assessment No. 121 (Prepared by the Minnesota Evidence-based Practice Center under Contract No. 290-02-0009.) AHRQ Publication No. 05-E017-2. Rockville, MD.: Agency for Healthcare Research and Quality, 2005

13 Enright P. Does screening for COPD by primary care physicians have the potential to cause more harm than good? Chest 2006; 129:833-835

14 Halpern MT. Effect of smoking characteristics on cognitive dissonance in current and former smokers. Addict Behav 1994; 19:209-217

15 McMaster C, Lee C. Cognitive dissonance in tobacco smokers. Addict Behav 1991; 16:349-353

16 Chapman S, Wong WL, Smith W. Self-exempting beliefs about smoking and health: differences between smokers and ex-smokers. Am J Public Health 1993; 83

17 Lee C. Perceptions of immunity to disease in adult smokers. Journal of Behavioral Medicine 1997; 12:267-277

18 McCoy SB, Gibbons FX, Reis TJ, et al. Perceptions of smoking risk as a function of smoking status. J Behav Med 1992; 15:469-488

19 Peters JM, Ferris BG, Jr. Smoking, pulmonary function, and respiratory symptoms in a college-age group. Am Rev Respir Dis 1967; 95:774-782

20 Kotz D, Huibers MJ, Vos R, et al. Principles of confrontational counselling in smokers with chronic obstructive pulmonary disease (COPD). Med Hypotheses 2008; 70:384-386

21 Fiore MC, Bailey WC, Cohen SJ, et al. Treating tobacco use and dependence. Clinical Practical Guideline. Rockville, MD: US Department of Health and Human Services, 2000

22 Pieterse ME, Seydel ER, DeVries $H$, et al. Effectiveness of a minimal contact smoking cessation program for Dutch general practitioners: a randomized controlled trial. Prev Med 2001; 32:182-190

23 Miller MR, Hankinson J, Brusasco V, et al. Standardisation of spirometry. Eur Respir J 2005; 26:319-338

24 Miller MR, Crapo R, Hankinson J, et al. General considerations for lung function testing. Eur Respir J 2005; 26:153-161

25 Zelen M. A new design for randomized clinical trials. N Engl J Med 1979; 300:1242-1245

26 Zelen M. Randomized consent designs for clinical trials: an update. Stat Med 1990; 9:645-656 
27 Torgerson DJ, Roland M. What is Zelen's design? BMJ 1998; 316:606

28 STIVORO. De L-MIS: stoppen met roken voor longpatiënten. Handleiding voor longverpleegkundigen. [The L-MIS: smoking cessation in lung patients. Strategy for respiratory nurses]. Den Haag: STIVORO, 2003

29 Fletcher C, Peto R. The natural history of chronic airflow obstruction. Brit Med J 1977; 1:1645-1648

30 Hughes JR, Stead LF, Lancaster T. Antidepressants for smoking cessation. Cochrane Database Syst Rev 2004

31 Lancaster T, Stead LF. Individual behavioural counselling for smoking cessation. Cochrane Database Syst Rev 2004

32 Silagy C, Lancaster T, Stead LF, et al. Nicotine replacement therapy for smoking cessation. Cochrane Database Syst Rev 2004

33 Tonnesen P, Carrozzi L, Fagerstrom KO, et al. Smoking cessation in patients with respiratory diseases: a high priority, integral component of therapy. ERS task force guideline. Eur Respir J 2007; 29:390-417

34 Wagena EJ, Knipschild P, Zeegers MPA. Should nortriptyline be used as a first-line aid to help smokers quit? Results from a systematic review and meta-analysis. Addiction 2005; 100:317-326

35 Langone JJ, Gjika HB, Van Vunakis H. Nicotine and its metabolites. Radioimmunoassays for nicotine and cotinine. Biochemistry 1973; 12:5025-5030

36 SRNT Subcommittee on Biochemical Verification. Biochemical verification of tobacco use and cessation. Nicotine Tob Res 2002; 4:149-159

37 Goossens MEJB, Molken MPMHR-v, Vlaeyen JWS, et al. The cost diary: a method to measure direct and indirect costs in cost-effectiveness research. Journal of Clinical Epidemiology 2000; 53:688-695

38 The EuroQol Group. EuroQol--a new facility for the measurement of health-related quality of life. Health policy Amsterdam, Netherlands 1990; 16:199-208

39 Brooks R, the EuroQol Group. EuroQol: the current state of play. Health Policy 1996; 37:53-72

40 Mudde AN, Willemsen MC, Kremers S, et al. Meetinstrumenten voor onderzoek naar stoppen met roken. [Measurements for research on smoking cessation.]. Den Haag: Stivoro, 2000

41 Heatherton TF, Kozlowski LT, Frecker RC, et al. The Fagerström Test for Nicotine Dependence: a revision of the Fagerström Tolerance Questionnaire. Br J Addict 1991; 86:1119-1127

42 Price DB, Tinkelman DG, Nordyke RJ, et al. Scoring System and Clinical Application of COPD Diagnostic Questionnaires. Chest 2006; 129:1531-1539

43 Price DB, Tinkelman DG, Halbert RJ, et al. Symptom-based questionnaire for identifying chronic obstructive pulmonary disease in smokers. Respiration 2006; 73:285-295

44 Bestall JC, Paul EA, Garrod R, et al. Usefulness of the Medical Research Council (MRC) dyspnoea scale as a measure of disability in patients with chronic obstructive pulmonary disease. Thorax 1999; 54:581-586

45 Fletcher CM. Standardised questionnaire on respiratory symptoms: a statement prepared and approved by the MRC Committee on the Aetiology of Chronic Bronchitis (MRC breathlessness score). BMJ 1960; 2:1665

46 van der Molen T, Willemse BWM, Schokker S, et al. Development, validity and responsiveness of the Clinical COPD Questionnaire. Health and Quality of Life Outcomes 2003; 1

47 van der Molen T, Willemse BW, Schokker S, et al. Development \& cross sectional validity of the COPD symptom control questionnaire. Prim Care Resp J 2001; 10:75

48 Ware JE, Jr., Sherbourne CD. The MOS 36-item short-form health survey (SF-36). I. Conceptual framework and item selection. Med Care 1992; 30:473-483

49 McHorney CA, Ware JE, Raczek AE. The MOS 36-item short-form health survey (SF-36): II. Psychometric and clinical tests of validity in measuring physical and mental health constructs. Med Care 1993; 31:247-263

50 Williams JEA, Singh SJ, Sewell L, et al. Development of a self-reported Chronic Respiratory Questionnaire (CRQ-SR). Thorax 2001; 56:954-959

51 Guyatt GH, Berman LB, Townsend M, et al. A measure of quality of life for clinical trials in chronic lung disease. Thorax 1987; 42:773-778

52 Beck AT, Ward $\mathrm{CH}$, Mendelson M, et al. An inventory for measuring depression. Arch Gen Psychiatry $1961 ; 4: 561-571$

53 Zigmond AS, Snaith RP. The Hospital Anxiety and Depression Scale. Acta Psyciatr Scan 1983; 67:361-370

54 Spinhoven PH, Ormel J, Sloekers PPA, et al. A validation of the Hospital Anxiety and Depression Scale (HADS) in different groups of Dutch subjects. Psychological Medicine 1997; 27:363-370

55 Bjelland I, Dahl AA, Tangen Haug T, et al. The validity of the Hospital Anxiety and Depression Scale. An updated literature review. J Psychosom Res 2002; 52:69-77 



\title{
Chapter 6
}

\section{External validation of a chronic obstructive pulmonary disease diagnostic questionnaire}

\author{
Daniel Kotz ${ }^{1}$ \\ Patty Nelemans ${ }^{2}$ \\ Onno C.P. van Schayck ${ }^{1}$ \\ Geertjan Wesseling ${ }^{3}$
}

Published in: European Respiratory Journal 2008; 31 (2): 298-303

${ }^{1}$ Department of General Practice, School for Public Health and Primary Care (CAPHRI), Maastricht University Medical Centre, Maastricht, the Netherlands. ${ }^{2}$ Department of Epidemiology, Maastricht University Medical Centre, Maastricht, the Netherlands. ${ }^{3}$ Department of Respiratory Medicine, Maastricht University Medical Centre, Maastricht, the Netherlands 


\section{ABSTRACT}

\section{Background}

The aim of the present study was to determine the external validity of a recently developed questionnaire for identifying patients at increased risk of airflow limitation in smokers from the general population in Dutch- and Belgian-Limburg.

\section{Methods}

As part of a study on early detection of airflow limitation and subsequent smoking cessation treatment (ISRCTN 64481813), the COPD diagnostic questionnaire developed by Price et al. was used in current smokers aged 40 - 70 years, with a smoking history of 10 or more pack years, who reported one or more respiratory symptoms (cough, sputum production, or dyspnoea) but who had no diagnosis of a respiratory disease (COPD, asthma). Spirometry according to ATS/ERS criteria served as a reference test.

\section{Results}

Six hundred seventy-six subjects entered the analyses. Of these, 398 had normal lung function and 278 had a diagnosis of COPD (post-bronchodilator $\mathrm{FEV}_{1} / \mathrm{FVC}<0.70$ ). The ability of the COPD diagnostic questionnaire to discriminate between subjects with and without COPD was poor: $\mathrm{ROC}_{\mathrm{AUC}}=0.65$.

\section{Conclusions}

In a high risk population consisting of middle-aged current smokers with more than 10 pack years, the COPD diagnostic questionnaire is probably not useful as a diagnostic tool to identify patients with an increased risk of airflow limitation. 


\section{INTRODUCTION}

Chronic obstructive pulmonary disease (COPD) is associated with high personal and societal burden and mortality. Projections for 2020 indicate further increase in global COPD mortality, placing COPD on the $3^{\text {rd }}$ position of lethal diseases. ${ }^{1}$ Underdiagnosis of COPD is a worldwide problem. ${ }^{2}$ Most patients present to their doctor for various other reasons but often have respiratory symptoms, and in those who do present with respiratory symptoms, COPD is not always diagnosed. ${ }^{3}$ Because of the irreversible and progressive nature of the disease, early identification of COPD and subsequent treatment is important. However, widespread spirometric testing for early detection without pre-selection of patients at risk may result in wasting of healthcare resource utilization. ${ }^{4,5}$

The efficiency of early detection of COPD can be enhanced (in terms of detection rate and accuracy) by using simple self-administered questionnaires to identify persons in whom airflow limitation is likely. ${ }^{6,7}$ Price et al. have developed a new symptom-based questionnaire for identifying patients at increased risk of airflow limitation. ${ }^{8,9}$ Their "COPD diagnostic questionnaire" discriminates between subjects with and without airflow limitation. The authors concluded that their questionnaire could be used to identify patients with a high likelihood of having airflow limitation and that combining it with spirometry could help to improve the efficiency and accuracy of COPD diagnosis in primary care. ${ }^{8,9}$ The questionnaire has recently been implemented in the guideline for chronic airways diseases of the International Primary Care Airways Group (IPAG). ${ }^{10}$ The guideline recommends to use the questionnaire in current and former smokers aged 40 years or older who present with respiratory symptoms but with no prior history of respiratory disease or current regular respiratory treatment.

Before a new diagnostic questionnaire can be accepted and applied reliably in clinical practice, the analysis of the underlying model must be repeated on new data collected from an appropriate sample of subjects from a different setting. ${ }^{11-13}$ This process is called external validation. The aim of the present study was to determine the validity of the COPD diagnostic questionnaire in current smokers from the general population in Dutch- and Belgian-Limburg.

\section{METHODS}

\section{Development of the COPD diagnostic questionnaire in the original sample}

The development of the COPD diagnostic questionnaire has been described in detail elsewhere. ${ }^{8,9}$ In brief, 818 participants completed a list of questions and underwent spirometry. COPD was defined according to the Global Initiative for Chronic Obstruc- 
tive Lung Disease (GOLD) guideline ${ }^{14}$ by post-bronchodilator (post-bd.) $\mathrm{FEV}_{1} / \mathrm{FVC}<0.70$. Multiple logistic regression models were constructed to identify eight items to discriminate between persons with and without COPD (these items are listed in table 2). The scoring system of the questionnaire allows to calculate an overall COPD risk based on the weighted scores of these items. The sum score ranges from 0 to 38 points. According to the manual of the questionnaire, subjects can be classified as being at "high" (>19.5 points), "moderate" (16.5 - 19.5 points) or "low" (0 16.5 points) risk of COPD. The area under the receiver operating characteristics curve $\left(R_{A O C}\right)$ was 0.82 . The diagnostic odds ratios (DOR) for the two cut-off points 16.5 and 19.5 were $\mathrm{DOR}=5.5$ and $\mathrm{DOR}=4.8$ (calculation based on Price et al. ${ }^{9}$ ).

\section{External validation sample}

This external validation study was part of a study on early detection of airflow limitation and subsequent smoking cessation treatment. The trial protocol was approved by the medical ethics committee of Maastricht University / Maastricht University Hospital and registered at the Netherlands Trial Register (ISRCTN 64481813).

Subjects were recruited from the general population (through advertisements in local newspaper, flyers, posters, and mailings to households) and from primary care practices (during consultations and through posters and personalized mailings) in Dutch- and Belgian-Limburg (the region surrounding Maastricht), in the period from January 2005 through December 2006. Smokers aged 35 through 70 years, who were current smokers but motivated to quit smoking, were invited to take part in a study on individual counselling and medication for smoking cessation. The external validation of the COPD diagnostic questionnaire was performed in current smokers aged 40 through 70 years.

Eligibility was screened during an initial telephonic interview. Inclusion criteria were: smoking history of 10 or more pack years; being motivated to stop smoking; being competent to read and speak Dutch; and reporting a respiratory symptom, defined as an affirmative answer to at least one of the following three questions: "Do you cough regularly?", "Do you cough up phlegm (sputum) when you don't have a cold?" or "Have you been shorter of breath lately?". Exclusion criteria were: evidence of a prior respiratory diagnosis, defined by an affirmative answer to the question "Do you have COPD, chronic bronchitis, asthma or asthmatic bronchitis?". They were also not allowed to have undergone a lung function test (spirometry) during the preceding 12 months. As candidates were screened for taking part in a trial on smoking cessation, one or more contraindications for using the smoking cessation medication (nortriptyline) were also criteria for exclusion, among others the current use of antidepressants. Nortriptyline is a tricyclic anti-depressant which should not be used for smoking cessation in conjunction with another anti-depressant. 
After the initial telephonic interview, the participant information with the informed consent form and the COPD diagnostic questionnaire were sent to eligible subjects, and a date was fixed for spirometry. Linguistic validation of the originally English COPD diagnostic questionnaire for use in Dutch speaking people had been performed by an experienced international research institute (Mapi Research Institute). This process comprised conceptual definition of items, forward and backward translation, and testing. Subjects filled out the questionnaire at home and handed it in during the spirometry visit where they also signed the informed consent form.

Spirometry was performed by two qualified research assistants under permanent supervision of a pulmonologist (GJW) according to the criteria of the American Thoracic Society (ATS) / European Respiratory Society (ERS) task force for standardization of lung function testing ${ }^{15,16}$ using a Vitalograph ${ }^{\circledR} 2120$ (Vitalograph Ltd, Buckingham, England). After a minimum of three acceptable and reproducible FVC manoeuvres, a bronchodilator (500 $\mu$ g terbutaline) was administered to the subject in preparation for the reversibility test. After 15 minutes, another series of three FVC manoeuvres was performed. All spirometric test results were independently validated by a pulmonologist (GJW) and by a specialised lung function laboratory assistant who was not involved in the trial. Both were blinded for the scores on the COPD questionnaire. In case of initial disagreement, consensus was obtained during reexamination. Like in the study of Price et al. ${ }^{8,9}$, a diagnosis of COPD was confirmed by post-bd. $\mathrm{FEV}_{1} / \mathrm{FVC}<0.70$, according to the international GOLD guideline. ${ }^{14}$

\section{Statistical analyses}

We calculated the sum score on the COPD questionnaire based on the original scoring system. ${ }^{9}$ We then calculated the $\mathrm{ROC}_{\mathrm{AUC}}$, sensitivity, specificity, and DOR for the two cut-off points that are presented in the questionnaire manual. We fitted a multiple logistic regression model including the 8 items from the COPD diagnostic questionnaire as independent variables and COPD as dependent variable to compare odds ratios, logistic regression coefficients and corresponding P-values between the original sample and the external validation sample. All analyses were performed using SPSS (version 13.0).

\section{RESULTS}

\section{Study population}

A summary of the study enrolment is shown in figure 1. A total number of 1,711 subjects were screened for eligibility at the telephone. The majority, 1,209 subjects (70\% of 1,711$)$, had responded to an advertisement. One hundred and sixty-six $(10 \%)$ had been motivated by their GP to take part in the study, $196(11 \%)$ by a 
related study participant and $140(8 \%)$ responded to flyers and posters, or their motivation was not recorded. One thousand and fifty-two subjects were found eligible and were invited for spirometry. Spirometry was performed and the COPD diagnostic questionnaire was collected in 826 subjects; the remaining 226 subjects cancelled their spirometry appointment beforehand or did not show up. Thirteen percent of the spirometric test results (110/826) were found invalid and had to be excluded from the analyses. This proportion of invalid test results was higher than in the original study $(80 / 898=9 \%)$. The 110 subjects with invalid test results did not differ from subjects with valid test results with regard to sex, age, pack years and sum score on the COPD diagnostic questionnaire (results of the statistical tests not reported). Forty subjects were excluded because they had a missing value on one or more of the items of the COPD diagnostic questionnaire. The characteristics of these subjects did not differ from subjects with complete data on the COPD diagnostic questionnaire except for the post-bd. FVC, which was significantly lower: 3.51 Liter (standard deviation $(S D)=0.76$ ) compared to 3.98 Liter $(S D=0.95 ; p=0.002)$. Complete valid data from 676 subjects entered the analyses.

\section{Characteristics of the external validation sample}

Three hundred and ninety-eight subjects had a normal lung function (59\% of 676 ; mean post-bd. $\mathrm{FEV}_{1} / \mathrm{FVC}=77.9$, mean post-bd. $\mathrm{FEV}_{1} \%$ predicted=94.1) and 278 subjects had a diagnosis of COPD (41\%; mean post-bd. $\mathrm{FEV}_{1} / \mathrm{FVC}=61.9$, mean postbd. $\mathrm{FEV}_{1} \%$ predicted=79.7). In the latter group, airflow limitation according to GOLD criteria ${ }^{14}$ was mild in 142 (21\% of 676$)$, moderate in $119(18 \%)$ and severe to very severe in $17(3 \%)$ subjects. All other characteristics are shown in table 1 . On average, subjects from our external validation sample were younger and had slightly worse lung function parameters than subjects from the original sample. The prevalence of COPD was much higher ( $41 \%$ versus $19 \%)$. All subjects were current smokers, whereas only $45 \%$ of the original sample were current smokers. This difference in smoking status probably explains the large difference in mean pack years (40 versus 26 in the original sample). We split the external validation sample into two subsets according to the study diagnosis to compare differences in characteristics. This is also shown in table one (without calculating statistical differences). Smokers with COPD were more likely to be older, male, and to have more pack years.

\section{Discriminative ability of the COPD diagnostic questionnaire}

When applying the two cut-off points from the questionnaire manual (16.5 points for a low risk and 19.5 points for a high risk), 127 subjects (19\% of 676) were categorized as having a low risk of COPD, $183(27 \%)$ as having a moderate risk and 366 (54\%) as having a high risk of COPD. Within these three risk categories, the observed prevalence of the COPD was 24\% (30/127 subjects) in the low risk category, $36 \%(65 / 183)$ in the moderate risk category and 50\% $(183 / 366)$ in the high risk 
category. The ability of the COPD diagnostic questionnaire to discriminate between subjects with and without the COPD is graphically shown in the ROC curve of figure 2. The solid line represents levels of sensitivity and false positive rates for all cut-off points on the sum score of the questionnaire. The area under the ROC curve was considerably lower $\left(R O R_{A U C}=0.65\right)$ than in the original sample $\left(R_{O A C C}=0.82\right)$. Sensitivity (SN), specificity (SP) and diagnostic odds ratio (DOR) for the cut-off point 16.5 were $\mathrm{SN}=89.2 \%, \mathrm{SP}=24.4 \%$ and $\mathrm{DOR}=2.67$. For the cut-off point 19.5 , these parameters were $\mathrm{SN}=65.8 \%, \mathrm{SP}=54.0 \%$ and $\mathrm{DOR}=2.26$.

\section{Refit of the multiple logistic regression model}

The results of the multiple logistic regression analysis which modelled the probability of having COPD versus not having COPD are shown in table 2. Statistically significant associations were observed for age, the highest BMI category, and for the two items "phlegm in the morning" and "any wheeze". The other items were associated with odds ratios that were not significantly different from 1 .

As age seemed to be the most important predictor of the disease, we calculated another ROC curve of age as a single factor for predicting COPD. This curve is also shown in figure 2, as the dashed line. The area under that ROC curve was 0.67, meaning that the variable age alone had similar discriminative ability as the sum score on the COPD case finding questionnaire in our sample.

\section{DISCUSSION}

In the present study, we tested the validity of a recently developed COPD diagnostic questionnaire in 676 current smokers from the general population in Dutch- and Belgian-Limburg. The discriminative ability of the questionnaire was poor $\left(R_{A U C}=0.65\right)$.

The COPD diagnostic questionnaire was developed to improve the efficiency and accuracy of COPD diagnosis in primary care by discriminating between subjects with and without airflow limitation. 8,9 When applying the cut-off points from the manual, the questionnaire reached a sensitivity from $65.8 \%$ (at cut-off point of 19.5 ) to $89.2 \%$ (at cut-off point of 16.5 ). However, the corresponding specificity was only $54.0 \%$ and $24.4 \%$. This means that at cut-off point of 16.5 , almost nine out of ten subjects with airflow limitation were correctly identified by the questionnaire (true positive). But at the same time, almost three quarters of subjects without airflow limitation were incorrectly classified by the questionnaire as having COPD (false positive). A diagnostic test that is intended to discriminate between subjects with and without a disease should combine high levels of sensitivity and specificity. 
The area under the receiver operating characteristics curve, however, was very low: $\mathrm{ROC}_{\mathrm{AUC}}=0.65\left(\mathrm{ROC}_{\mathrm{AUC}}=0.50\right.$ indicates a totally uninformative test $\left.{ }^{11}\right)$. The combination of the 8 items of the COPD diagnostic questionnaire did not perform better than the item age alone $\left(\mathrm{ROC}_{\mathrm{AUC}}=0.67\right)$. Another indicator for the discriminative ability of the questionnaire, the diagnostic odds ratio, was also very low: $\mathrm{DOR}=2.67$ at the cut-off point of 16.5 . The value of the DOR ranges from 0 to infinity, with value 1 meaning that a test does not discriminate between subjects with the disease under study and those without it. ${ }^{17}$

Why does the COPD diagnostic questionnaire show such a low external validity? It is not unusual that newly developed diagnostic questionnaires perform more poorly when evaluated in an external sample of subjects from a different setting. An important reason for the poor performance of the COPD diagnostic questionnaire is that our sample differs from the original sample. This external validation was part of a study on early detection of smokers with airflow limitation and subsequent smoking cessation treatment. Subjects from our sample were all current smokers with at least 10 pack years (mean=40 pack years) of smoking history. We included only current smokers because smoking is by far the most important risk factor for COPD, and smoking cessation is the single most effective way to affect the outcome in patients who have been positively screened. ${ }^{18,19}$ In the sample of Price et al. ${ }^{8,9}$, only $45 \%$ were current smokers and the mean number of pack years was much lower (26 pack years). An explanation for the difference in performance may be the difference in smoking status between our external validation sample and the original sample. We suggest that the COPD diagnostic questionnaire discriminates between current smokers and former or non-smokers, rather than between subjects with and without airflow limitation. Previous research has shown that smoking has acute effects on respiratory symptoms. For example, in the Nord-Trøndelag Health Study, which is a population based study of more than 65,000 subjects, a significantly higher proportion of smokers compared to ex-smokers reported symptoms of wheezing, breathlessness, daily coughing, and coughing with phlegm. ${ }^{20}$ Respiratory symptoms were very common among smokers from our study as well: $91 \%$ of the subjects initially screened reported one of the three symptoms: cough, sputum production or shortness of breath, only $9 \%$ had none of these three symptoms. It is not likely that exclusion of this small proportion of subjects from the present study led to a selective study sample. As smoking is so closely correlated with the presence of respiratory symptoms, we think that the development and use of a diagnostic questionnaire for COPD should be done in current and former smokers separately. In the end, such a questionnaire is not intended to discriminate between the presence or absence of respiratory symptoms, but to discriminate between having COPD or not having COPD. It would be interesting to re-analyse the performance of the questionnaire in the two subgroups of smokers and former smokers from the original dataset. 
Apart from the smoking status, subjects from our external validation sample were younger and had slightly worse lung function parameters than subjects from the original sample. Furthermore, the proportion of invalid test results was higher $(17 \%$ versus $9 \%$ ). This may be due to a higher measurement error, or to more conservative criteria for the assessment of the quality. Nevertheless, it is unlikely that the exclusion of subjects with invalid test results affected the results as subjects with invalid test results had similar characteristics when compared to subjects with valid test results. The prevalence of COPD was much higher in our sample $(41 \%)$ than in the original sample (19\%). However, it is not likely that this higher prevalence affected the discriminative ability of the questionnaire because ROC and DOR are not dependent of the prevalence of disease (although they are influenced by the spectrum of disease severity). ${ }^{17,21}$

Based on the results of this external validation study we conclude that the COPD diagnostic questionnaire is probably not useful as a diagnostic tool to identify patients at increased risk of airflow limitation among current smokers. The questionnaire does not discriminate between subjects with and without airflow limitation in a high risk population (i.e. in middle-aged current smokers with more than 10 pack years). This study highlights the importance of external validation of a newly developed diagnostic instrument prior to the implementation in guidelines and daily practice. 


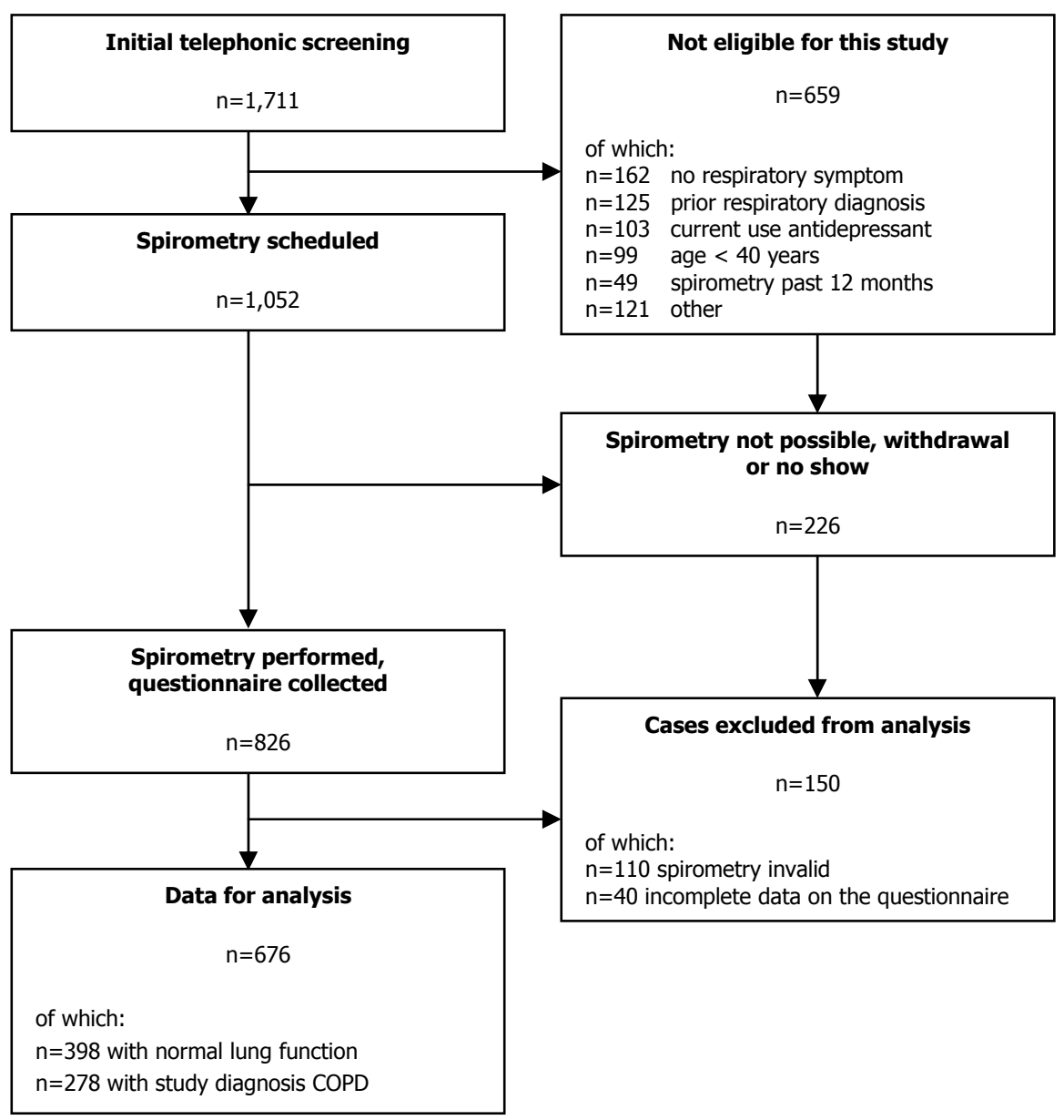

Figure 1: Study enrolment. 
Table 1: Comparison of characteristics between the original sample and the external validation sample

\begin{tabular}{|c|c|c|c|c|}
\hline \multirow[t]{2}{*}{ Characteristics } & \multirow{2}{*}{$\begin{array}{l}\text { Original sample } \\
\qquad n=818\end{array}$} & \multicolumn{3}{|c|}{ External validation sample } \\
\hline & & $\begin{array}{c}\text { Total sample } \\
n=676\end{array}$ & $\begin{array}{c}\text { No COPD } \\
n=398\end{array}$ & $\begin{array}{l}\text { COPD" } \\
n=278\end{array}$ \\
\hline Years of age & $58.2(11.2)$ & $52.3(7.3)$ & $50.6(6.9)$ & $54.7(7.0)$ \\
\hline Male sex, N (\%) & $403(49.3)$ & $397(58.7)$ & $225(57.3)$ & $172(62.3)$ \\
\hline Body Mass Index, $\mathrm{kg} / \mathrm{m}^{2}$ & $28.3(5.7)$ & $25.4(4.2)$ & $25.5(4.2)$ & $25.2(4.1)$ \\
\hline Current smokers, N (\%) & $364(44.5)$ & $676(100)$ & $398(100)$ & $278(100)$ \\
\hline Pack years $^{\dagger}$ & $25.6(24.3)$ & $40.4(19.3)$ & $38.1(19.1)$ & $43.6(19.0)$ \\
\hline FTND $^{\neq}$ & not reported & $4.45(1.4)$ & $4.39(1.4)$ & $4.53(1.5)$ \\
\hline $\mathrm{FEV}_{1}$ post-bd. $\%$ pred. & $94.4(17.0)$ & $88.2(16.4)$ & $94.1(11.9)$ & $79.7(18.1)$ \\
\hline FVC post-bd. \% pred. & $95.8(15.5)$ & $101.4(15.8)$ & $99.9(13.7)$ & $103.6(18.2)$ \\
\hline FEV $_{1} /$ FVC post-bd. $\%$ pred. & $98.2(9.7)$ & $89.5(11.9)$ & $97.5(6.0)$ & $78.1(8.3)$ \\
\hline
\end{tabular}

Data are presented as mean (SD), unless otherwise indicated. ${ }^{\S}$ Data extracted from Price et al. (Respiration 2006; 73:285-295); ${ }^{\dagger} 1$ pack year $=$ number of cigarettes smoked per day $\mathrm{x}$ number of years smoking / 20; ${ }^{\ddagger}$ Fagerström Test for Nicotine Dependence: range from 0 (lowest level of nicotine dependence) through 10 (highest); "COPD is defined as post-bronchodilator (post-bd.) $\mathrm{FEV}_{1} / \mathrm{FVC}<0.70$ according to the Global Initiative for Chronic Obstructive Lung Disease (GOLD) 2006 guideline
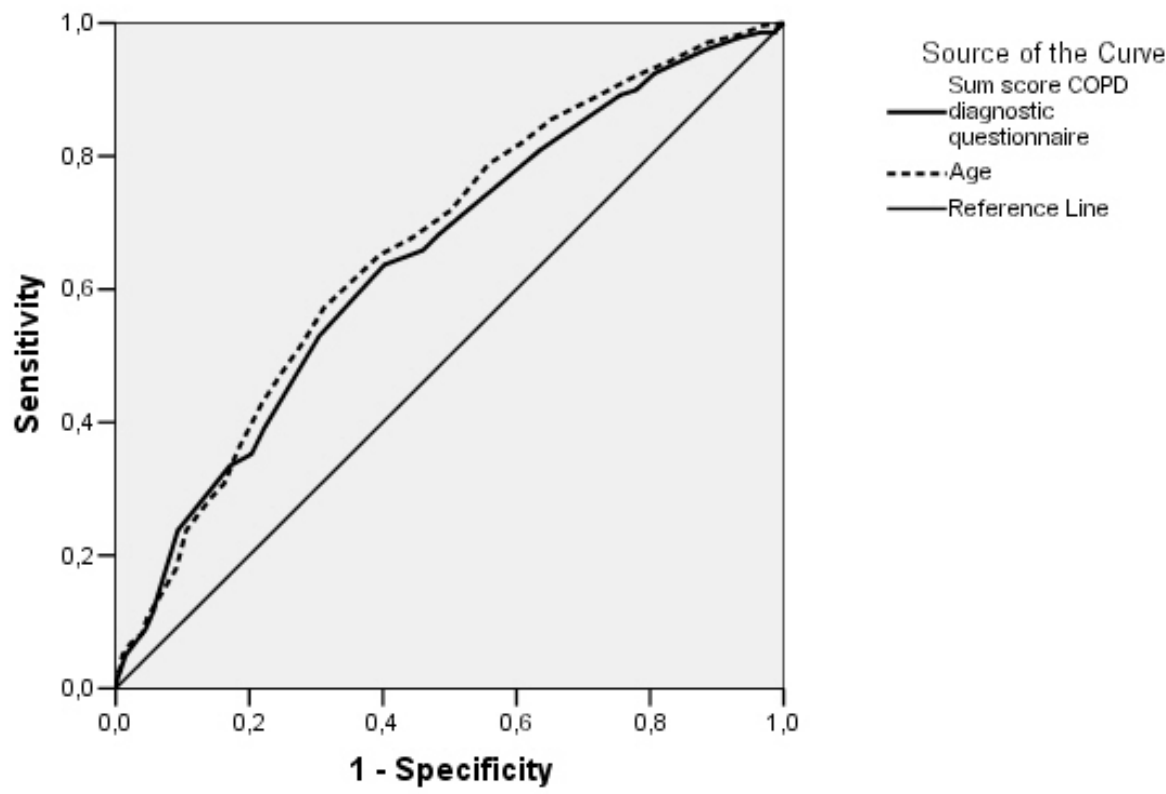

Figure 2: ROC curves of predictors of COPD in the external validation sample. The solid line represents the sum score of the COPD diagnostic questionnaire (area under the solid ROC curve $=0.65$ ). The dashed line represents age only (area under the dashed ROC curve $=0.67$ ). 
Table 2: Comparison of multiple regression models between the original sample and the external validation sample

\begin{tabular}{|c|c|c|c|c|c|c|c|}
\hline \multirow[t]{3}{*}{ Items } & \multicolumn{2}{|c|}{ Original sample ${ }^{\S}(n=798)$} & \multicolumn{5}{|c|}{ External validation sample $(n=676)$} \\
\hline & \multirow[t]{2}{*}{$\mathrm{OR}^{+}$} & \multirow[t]{2}{*}{ P-value } & \multirow[t]{2}{*}{$\mathrm{n}$} & \multirow[t]{2}{*}{$\mathrm{OR}^{+}$} & \multicolumn{2}{|c|}{$95 \%$ CI for OR } & \multirow{2}{*}{ P-value } \\
\hline & & & & & lower & upper & \\
\hline \multicolumn{8}{|l|}{ Age group, years } \\
\hline 40-49 (reference) & 1.00 & & 276 & 1.00 & & & \\
\hline $50-59$ & 2.20 & 0.022 & 265 & 1.99 & 1.38 & 2.88 & $<0.001$ \\
\hline $60-69$ & 4.73 & $<0.001$ & 133 & 2.92 & 1.85 & 4.61 & $<0.001$ \\
\hline$>=70$ & 7.77 & $<0.001$ & 2 & n.a.* & n.a.* & n.a.* & n.a.* \\
\hline \multicolumn{8}{|l|}{ Body Mass Index, $\mathrm{kg} / \mathrm{m}^{2}$} \\
\hline$<25.4$ (reference) & 1.00 & & 386 & 1.00 & & & \\
\hline 25.4-29.7 & 0.44 & 0.002 & 191 & 0.66 & 0.45 & 0.97 & 0.033 \\
\hline$>29.7$ & 0.35 & $<0.001$ & 99 & 0.57 & 0.35 & 0.94 & 0.026 \\
\hline \multicolumn{8}{|l|}{ Pack-years $^{\ddagger}$} \\
\hline$<15$ (reference) & 1.00 & & 26 & 1.00 & & & \\
\hline $15-24$ & 1.63 & 0.112 & 106 & 0.74 & 0.29 & 1.92 & 0.537 \\
\hline $25-49$ & 1.99 & 0.010 & 367 & 1.11 & 0.46 & 2.63 & 0.822 \\
\hline$>=50$ & 4.05 & $<0.001$ & 177 & 1.66 & 0.67 & 4.10 & 0.271 \\
\hline $\begin{array}{l}\text { Weather affects cough, } \\
\text { yes (versus no) }\end{array}$ & 1.68 & 0.089 & 138 & 0.87 & 0.58 & 1.31 & 0.502 \\
\hline $\begin{array}{l}\text { Phlegm without a cold, } \\
\text { yes (versus no) }\end{array}$ & 1.81 & 0.013 & 486 & 1.47 & 0.97 & 2.22 & 0.068 \\
\hline $\begin{array}{l}\text { Phlegm in the morning, } \\
\text { yes (versus no) }\end{array}$ & 0.54 & 0.022 & 253 & 0.67 & 0.46 & 0.99 & 0.045 \\
\hline $\begin{array}{l}\text { Wheeze, any (versus } \\
\text { never) }\end{array}$ & 2.08 & 0.001 & 535 & 1.74 & 1.14 & 2.68 & 0.011 \\
\hline $\begin{array}{l}\text { Present or previous } \\
\text { allergies, yes (versus no) }\end{array}$ & 0.52 & 0.005 & 154 & 0.98 & 0.67 & 1.47 & 0.994 \\
\hline
\end{tabular}




\section{REFERENCES}

1 Murray CJ, Lopez AD. Alternative projections of mortality and disability by cause 1990-2020: Global Burden of Disease Study. Lancet 1997; 349:1498-1504

2 Pauwels PRA, Rabe KF. Burden and clinical features of chronic obstructive pulmonary disease (COPD). Lancet 2004; 364:613-620

3 van Schayck CP, van der Heijden FMMA, van den Boom G, et al. Underdiagnosis of asthma: is the doctor or the patient to blame? The DIMCA project. Thorax 2000; 55:562-565

4 Wilt TJ, Niewoehner D, Kim C-B, et al. Use of Spirometry for case finding, diagnosis, and management of chronic obstructive pulmonary disease (COPD). Evidence Report/Technology Assessment No. 121 (Prepared by the Minnesota Evidence-based Practice Center under Contract No. 290-02-0009.) AHRQ Publication No. 05-E017-2. Rockville, MD.: Agency for Healthcare Research and Quality, 2005

5 Enright P. Does screening for COPD by primary care physicians have the potential to cause more harm than good? Chest 2006; 129:833-835

6 van Schayck CP, Loozen JM, Wagena $E$, et al. Detecting patients at a high risk of developing chronic obstructive pulmonary disease in general practice: cross sectional case finding study. BMJ 2002; 324:13701374

7 van Schayck CP, Halbert RJ, Nordyke RJ, et al. Comparison of existing symptom-based questionnaires for identifying COPD in the general practice setting. Respirology 2005; 10:323-333

8 Price DB, Tinkelman DG, Halbert RJ, et al. Symptom-based questionnaire for identifying chronic obstructive pulmonary disease in smokers. Respiration 2006; 73:285-295

9 Price DB, Tinkelman DG, Nordyke RJ, et al. Scoring System and Clinical Application of COPD Diagnostic Questionnaires. Chest 2006; 129:1531-1539

10 International Primary Care Airways Group (IPAG). Chronic airways diseases. A guide for primary care physicians., 2005

11 Knottnerus JA. The evidence base of clinical diagnosis. London: BMJ Books, 2002

12 Altman DG, Royston P. What do we mean by validating a prognostic model? Statistics in Medicine 2000; 19:453-473

13 Justice AC, Covinsky KE, Berlin JA. Assessing the generalizability of prognostic information. Ann Intern Med 1999; 130:515-524

14 Global Initiative for Chronic Obstructive Lung Disease. Global strategy for the diagnosis, management, and prevention of chronic pulmonary disease. Executive summary (http://www.goldcopd.com): NHLBI / WHO, 2006

15 Miller MR, Hankinson J, Brusasco V, et al. Standardisation of spirometry. Eur Respir J 2005; 26:319-338

16 Miller MR, Crapo R, Hankinson J, et al. General considerations for lung function testing. Eur Respir J 2005; 26:153-161

17 Glas AS, Lijmer JG, Prins MH, et al. The diagnostic odds ratio: a single indicator of test performance. Journal of Clinical Epidemiology 2003; 56:1129-1135

18 Anthonisen NR, Connett JE, Kiley JP, et al. Effects of smoking intervention and the use of an inhaled anticholinergic bronchodilator on the rate of decline of FEV1. The Lung Health Study. J Am Med Assoc 1994; 272:1497-1505

19 Pauwels RA, Buist AS, Calverley PM, et al. Global strategy for the diagnosis, management, and prevention of chronic obstructive pulmonary disease. NHLBI/WHO Global Initiative for Chronic Obstructive Lung Disease (GOLD) Workshop summary. Am J Respir Crit Care Med 2001; 163:1256-1276

20 Langhammer A, Johnsen R, Holmen J, et al. Cigarette smoking gives more respiratory symptoms among women than among men The Nord-Trondelag Health Study (HUNT). J Epidemiol Community Health 2000; 54:917-922

21 Altman DG. Practical statistics for medical research. London: Chapman \& Hall, 1991 



\title{
Chapter 7
}

\section{Confronting smokers with previously undetected COPD does not increase long-term smoking cessation rates}

\author{
Daniel Kotz ${ }^{1}$ \\ Geertjan Wesseling ${ }^{2}$ \\ Marcus J.H. Huibers ${ }^{3}$ \\ Onno C.P. van Schayck ${ }^{1}$
}

Submitted for publication

${ }^{1}$ Department of General Practice, School for Public Health and Primary Care (CAPHRI), Maastricht University Medical Centre, Maastricht, the Netherlands. ${ }^{2}$ Department of Respiratory Medicine, Maastricht University Medical Centre, Maastricht, the Netherlands. ${ }^{3}$ Department of Clinical Psychological Science, Maastricht University, Maastricht, the Netherlands. 


\section{ABSTRACT}

\section{Background}

The objective of the present study was to test whether confronting smokers with previously undetected chronic obstructive pulmonary disease (COPD) increases the rate of smoking cessation.

\section{Methods}

296 smokers with no prior diagnosis of COPD who were detected with mild to moderate airflow limitation by means of spirometry were randomly allocated to; medium intensity confrontational counselling delivered by a respiratory nurse combined with nortriptyline for smoking cessation (experimental group), medium intensity health education and promotion delivered by a respiratory nurse combined with nortriptyline (control group 1), or "care as usual" for smoking cessation by the general practitioner (control group 2). Only the experimental group was confronted with their abnormal spirometry.

\section{Results}

There was no difference in cotinine validated prolonged abstinence rate between the experimental group and control group 1 from week 5 through 52 (OR=0.96, $95 \% \mathrm{CI}=0.43,2.18)$.

\section{Conclusions}

The confrontational counselling approach did not increase the long-term abstinence from smoking rate compared with an equally intensive treatment in which participants were not confronted with spirometry. The high failure rates ( $88 \%$ and higher) highlight the need for treating tobacco addiction as a chronic relapsing disorder, especially in smokers with respiratory disease, who have a more urgent need to stop. 


\section{INTRODUCTION}

Chronic obstructive pulmonary disease (COPD) is a preventable and treatable disease which is characterized by airflow limitation that is not fully reversible. ${ }^{1}$ Spirometry is the gold standard for the diagnosis and assessment of the disease. ${ }^{1}$ COPD is currently the fifth leading cause of death worldwide ${ }^{2}$, and projections for 2020 indicate a further increase in global mortality, placing COPD in the third position of lethal diseases. ${ }^{3}$ Cigarette smoking is by far the most important risk factor for COPD, and smoking cessation is the single most effective way to reduce the risk of developing COPD and to affect the outcome in patients at all stages of the disease. ${ }^{4,5}$

Underdiagnosis of COPD is a worldwide problem. ${ }^{6}$ Most patients present to their doctor for various other reasons but often have respiratory symptoms, and in those who do present with respiratory symptoms, COPD is not always suspected nor diagnosed. ${ }^{7}$ Because of the irreversible and progressive nature of the disease, early intervention is important. However, the use of spirometry for early detection of airflow limitation and COPD is still an issue of debate. ${ }^{8-10}$ The most important counterargument is that there is no convincing evidence that spirometry increases smoking cessation rates. ${ }^{11-13}$

Discussing abnormal test results with smokers has been suggested as a "teachable moment" that may increase motivation to quit smoking, but there is only weak evidence to support such an approach. ${ }^{14}$ Various studies have been performed on the efficacy of spirometry as a motivational tool for smoking cessation but their results are inconclusive. ${ }^{11,12}$ Findings are often of limited validity because of one or more important biases such as unstandardized counselling intensity, incomparable or uncontrolled use of pharmacological aids for smoking cessation between experimental and control group, or different (or unclear) baseline levels of lung function and motivation to quit smoking. ${ }^{15}$ The most recent randomized trial clearly showed a positive effect; telling smokers their "lung age" (based on spirometry) increased the abstinence rate by $7.2 \%$ after 52 weeks. ${ }^{16}$

We hypothesised that early detection of COPD and confrontation with spirometry for smoking cessation may be effective if "confrontational counselling" is applied ${ }^{17}$. Confrontational counselling is a patient-centred approach which involves confronting smokers with the consequences of their addiction (previously undiagnosed COPD) and which uses specific communication skills to identify and challenge irrational beliefs about smoking. We conducted a randomized controlled trial to assess the efficacy of confrontational counselling in comparison with regular health education and promotion for smoking cessation delivered by specialized respiratory nurses in current smokers with previously undiagnosed mild to moderate airflow limitation, with regard to prolonged abstinence from smoking rates from week 5 through 52 
after the target quit date. Secondary outcomes were abstinence rates at week 5 and from week 5 through 26.

\section{METHODS}

The trial was designed to assess the "net" effect of confronting smokers with spirometry by comparing medium intensity confrontational counselling delivered by a respiratory nurse combined with nortriptyline for smoking cessation (experimental group) with medium intensity health education and promotion delivered by a respiratory nurse combined with nortriptyline for smoking cessation (control group 1). The effect of both treatments were compared with low intensity "care as usual" for smoking cessation by the general practitioner (control group 2). A detailed description of the protocol has been published elsewhere. ${ }^{18}$ The trial was approved by the medical ethics committee of Maastricht University Medical Centre and registered at the Netherlands Trial Register (ISRCTN 64481813).

\section{Recruitment and eligibility of participants}

Current smokers aged 35 through 70 years who were interested in quitting were recruited from the general population and from primary care practices in Dutch- and Belgian-Limburg (the region surrounding Maastricht). Eligibility was assessed during an initial telephone interview. Inclusion criteria were: smoking history of 10 or more pack years (= number of cigarettes smoked per day $\mathrm{x}$ number of years smoking / 20); being competent to read and speak Dutch; and reporting at least one of the respiratory symptoms: cough, sputum production, or shortness of breath. Exclusion criteria were: evidence of a prior respiratory diagnosis, defined by an affirmative answer to the question "Do you have COPD, chronic bronchitis, asthma or asthmatic bronchitis?". Subjects were also excluded if they had undergone spirometry during the preceding 12 months. One or more contraindications for using the smoking cessation medication (nortriptyline) were also criteria for exclusion, among others the current use of anti-depressants.

After the initial telephone interview, the participant information sheet with the informed consent form and the baseline questionnaire were sent to eligible subjects, and a date was fixed for spirometry at Medical Centre Annadal (Maastricht). The participant information sheet did not include any information about early detection and confrontation with COPD or the differences in counselling between the experimental and control group 1. The design we used was adapted from Zelen's de$\operatorname{sign}^{19,20}$ which may be particularly useful when evaluating the full unbiased impact of screening interventions. ${ }^{21}$ 
Spirometry was performed according to American Thoracic Society / European Respiratory Society criteria ${ }^{22,23}$ using a Vitalograph ${ }^{\circledR} 2120$ (Vitalograph Ltd, Buckingham, England). Final eligibility was determined if subjects had airflow limitation defined as post-bronchodilator (post-bd.) Forced Expiratory Volume in one second $\left(\mathrm{FEV}_{1}\right)$ / Forced Vital Capacity (FVC) $<70 \%$ in combination with post-bd. FEV $_{1} \geq 50 \%$ of predicted value; i.e. mild or moderate airflow limitation, according to the international Global Initiative for Chronic Obstructive Lung Disease (GOLD) guideline ${ }^{1}$. The results of spirometry were not discussed at that moment. Subjects with severe airflow limitation (post-bd. $\mathrm{FEV}_{1}<50 \%$ of predicted value) were excluded from participation and advised to contact their GP or a lung physician for further evaluation. Subjects without airflow limitation (post-bd. $\mathrm{FEV}_{1} / \mathrm{FVC}>70 \%$ ) were also excluded. All excluded smokers were told that despite their normal lung function, they still were at risk of getting other smoking related diseases which are not measured by spirometry, such as cancer or cardiovascular disease. They were strongly advised to give up smoking. After the last follow-up measurement, all participants received a debriefing letter with detailed information about the study and their GPs were informed about the results from spirometry.

All eligible subjects were contacted by telephone a few days after baseline spirometry to be randomised into one of the three intervention groups. The database of the trial incorporated a randomization system of seven participants per block, allowing an unequal group allocation of $3: 3: 1$; experimental group : control group 1 : control group 2 (seven participants per block ${ }^{\dagger}$.

\section{Interventions}

Participants from both the experimental group and control group 1 received medium intensity counselling delivered by a respiratory nurse combined with nortriptyline for smoking cessation. The common basis for the counselling in both groups was the socalled "L-MIS" protocol for the treatment of nicotine and tobacco addiction which had been implemented among all respiratory nurses in the Netherlands in previous years. ${ }^{24}$ The number of counselling sessions (4), their duration (40 minutes) and scheduling (weeks 1, 2, 3, and 4) was standardized in both treatment groups (table 1). Participants' attendance with counselling was assessed by counting the number of counselling sessions attended and dividing this number by 4 . Specific elements of "confrontational counselling"17 were added to the L-MIS in the experimental group, which discriminated the treatment from that in control group 1 (table 1). This involved discussing the results from spirometry and the prognosis of COPD and challenging irrational beliefs about smoking.

\footnotetext{
${ }^{+}$Note that we started out the trial with an equal group allocation of $1: 1: 1$ and than switched to $3: 3: 1$. This explains why the number of participants from control group 2 is higher than one third of the other two groups.
} 
Participants from the experimental group and control group 1 received an equal dosage of nortriptyline for smoking cessation. Nortriptyline is a tricyclic antidepressant which has been shown to be a cheap and effective alternative for the anti-depressant bupropion. ${ }^{25,26}$ Participants started taking nortriptyline on the day of the first counselling session (day 1 ). From day 1 through day 3, participants took one pill of $25 \mathrm{mg}$ nortriptyline once a day (preferably after dinner). From day 4 through day 7 , participants took $50 \mathrm{mg}$ a day (given as two pills of $25 \mathrm{mg}$ ). From day 8 through the end of the treatment period (day 49), participants took $75 \mathrm{mg}$ a day (given as three pills of $25 \mathrm{mg}$ ). The nurse monitored the correct use of the medication and the occurrence of side-effects. In case of unpleasant or severe side-effects, the dosage was reduced or the use of the medication was stopped.

To test whether the experimental intervention as a whole was more effective than primary care as usual, participants from control group 2 were referred to their own GP for smoking cessation treatment. They were asked to make an appointment with their GP within the next ten days. They were provided with a referral letter explaining to the GP that they were participating in a study on smoking cessation. This letter did not give any information about the results from spirometry and the fact that the participant had airflow limitation. The GP was asked to provide the care he/she usually provides to patients who want to quit smoking. In the Netherlands, primary care as usual for smoking cessation involves the use of a protocol for low intensity health education and promotion, the so-called "H-MIS"27. A semi-structured interview was used among participants from control group 2 during the first follow-up visit in order to assess whether participants had indeed consulted their GP and which treatment for smoking cessation the GP had delivered.

\section{Outcome measures}

The primary outcome measure was prolonged abstinence from smoking from week 5 to 52 after the target quit date. Prolonged abstinence was defined as urine cotinine validated $\left(<50 \mathrm{ng} / \mathrm{mL}^{28}\right.$ ) abstinence from smoking at all three follow-up visits; week 5,26 , and 52 .

Participants completed a questionnaire at baseline and at each follow-up visit. The questionnaire included various smoking characteristics and the Fagerström Test for Nicotine Dependence (FTND) ${ }^{29}$. Respiratory health complaints were measured with the Clinical COPD Questionnaire ${ }^{30,31}$. Health-related quality of life was measured with the Chronic Respiratory Questionnaire self-reported (CRQ) ${ }^{32,33}$.

\section{Data analyses}

Statistical differences in abstinence from smoking rates were analysed using simple logistic regression analyses to calculate odds ratios and $95 \%$ confidence intervals. As part of an ancillary analysis, we used multiple logistic regression models to adjust for 
baseline covariates that are known to be associated with the primary outcome (see for example: ${ }^{34}$ ); age, sex, level of education, number of previous quit attempts, anxiety (measured with the Hospital Anxiety and Depression Scale, HADS ${ }^{35}$ ), and nicotine addiction (measured with the FTND). All randomized subjects were included in the intention-to-treat analyses, and subjects not showing up at the follow-up visit or with a missing value on the measure of abstinence were regarded as smokers. The proportion of missing data on items from the questionnaire ranged between 0 $7 \%$. Missing data were not imputed for any analyses.

\section{RESULTS}

A total of 116 smokers with previously undetected COPD were randomly allocated to the experimental group, 112 to control group 1, and 68 to control group 2 (figure 1). After 52 weeks follow-up, the numbers of participants lost to follow-up (i.e. with no data on the primary outcome variable) were: $14(12 \%)$ in the experimental group, $19(17 \%)$ in control group 1 , and $15(22 \%)$ in control group 2 . The baseline characteristics are shown in table 2. A total of 160 participants (54\%) had mild COPD and $136(46 \%)$ had moderate COPD according to the GOLD classification.

\section{Treatment received}

One participant from the experimental group and one participant from control group 1 dropped out before the start of the counselling because they already had stopped smoking. Among the remaining participants, attendance in the counselling sessions was $95 \%$ in the experimental group and $92 \%$ in control group 1 (no statistically significant difference). The proportion of participants reporting one or more side effects of using nortriptyline to the respiratory nurse was $82 \%, 84 \%$, and $72 \%$ during the counselling sessions 2,3 , and 4 (no statistical significant difference between the groups). The mean number of side effects reported was lower in the experimental group (mean=1.4) than in control group 1 (mean=1.8; $p=0.017$ ). Among all participants reporting side effects, dry mouth was most frequently reported $(39 \%)$, followed by fatigue $(10 \%)$ and dizziness $(10 \%)$.

Of the 68 participants from control group 2 who were referred to their GP for care as usual for smoking cessation, 46 (68\%) actually consulted their GP, 4 (6\%) did not, and no information was available of 18 participants (27\%). Among the 46 participants who consulted their GP, the median number of consultations was 2 (maximum 5). The median duration of these consultations was 10 minutes (range from 5 to 45 minutes). Anti-depressants were prescribed for smoking cessation in 34 of the 46 participants; bupropion ( $\mathrm{N}=15)$, nortriptyline $(\mathrm{N}=17)$, or amitriptyline $(\mathrm{N}=1)$. Nicotine replacement therapy was prescribed in 6 of the 46 participants. 


\section{Abstinence from smoking}

The abstinence from smoking rates in the experimental group, control group 1 , and control group 2 dropped from 51\% ( $\mathrm{N}=59 / 116), 39 \%(\mathrm{~N}=44 / 112)$, and $18 \%$ $(\mathrm{N}=12 / 68)$ at week 5 after the target quit date to $11 \%(\mathrm{~N}=13 / 116), 12 \%(13 / 112)$, and $6 \%(4 / 68)$ from week 5 through 52 (table 3, figure 2). The odds of being abstinent from smoking was $60 \%$ higher in the experimental group than in control group 1 at week $5(\mathrm{OR}=1.60,95 \% \mathrm{CI}=0.95,2.70)$ and $43 \%$ higher from week 5 through 26 $(\mathrm{OR}=1.43,95 \% \mathrm{CI}=0.79,2.58)$. There was no difference in prolonged abstinence rates from week 5 through 52 ( $\mathrm{OR}=0.96,95 \% \mathrm{CI}=0.43,2.18$; table 3 ). The corresponding odds ratios adjusted for baseline covariates were $\mathrm{OR}=2.01$ ( $95 \% \mathrm{CI}=1.1$, 3.7) at week $5, \mathrm{OR}=1.58(95 \% \mathrm{CI}=0.82,3.03)$ from week 5 through 26 , and $\mathrm{OR}=0.88(95 \% \mathrm{CI}=0.38,2.03)$ from week 5 through 52 . Compared to control group 2 , the odds of being abstinent from smoking from week 5 through 52 was about twice as high in the experimental group and control group 1, but these differences were not statistically significant (table 3$)$. However, both at week $5(\mathrm{OR}=4.83)$ and from week 5 through $26(\mathrm{OR}=3.24)$, the odds of abstinence from smoking was significantly higher in the experimental group compared to control group 2.

\section{DISCUSSION}

We conducted a randomized controlled trial in 296 smokers with previously undetected mild to moderate COPD to assess the efficacy of confronting smokers with the results of spirometry for smoking cessation. Although we observed a clinically relevant but statistically non-significant difference at week 5 , the confrontational counselling approach did not increase the prolonged abstinence from smoking rate from week 5 through 52 compared with an equally intensive treatment in which participants were not confronted with spirometry. In both groups, the proportion of smokers that did not succeed to quit or relapsed into smoking were very high (about $88 \%)$.

The use of spirometry for early detection of COPD is an issue of debate, primarily because of a lack of convincing evidence that spirometry has an added positive effect on smoking cessation. ${ }^{11-13}$ The results from previous studies ${ }^{8,11,12}$ are inconclusive, but the most recent one ${ }^{16}$ shows a clear positive effect. Parkes et al. evaluated the impact of telling smokers their estimated lung age after spirometry. ${ }^{16}$ Contrary to our findings, statistically significantly more smokers from the intervention group than from the control group were abstinent from smoking after 52 weeks: $13.6 \%$ vs. $6.4 \%$. The authors concluded that "telling smokers their lung age significantly improves the likelihood of them quitting smoking". ${ }^{16}$ 
Why did Parkes et al. find an effect whereas we did not? We think this can be explained for a large part by the differences between the two studies in their recruitment strategy and resulting characteristics of the study samples. First of all, Parkes et al. mainly included smokers with normal lung function into their trial; only about $25 \%$ had abnormal lung function of which about one third were already known with COPD. In our study, we included only smokers with abnormal lung function and without a previous diagnosis of COPD. Thus, the two study samples are not comparable concerning their baseline risk of COPD, a factor that is likely to affect the treatment outcome. Furthermore, the recruitment strategy of Parkes et al. probably led to a selection of participants who were interested in their lung function and were therefore more susceptible to related health warnings. This is the same mechanism that may explain the results from a large observational study in smokers from Poland that showed that spirometry promoted cessation. ${ }^{36,37}$ In our study, participants responded to announcements for receiving a smoking cessation intervention (no attention was drawn to lung function testing). They may therefore have been less susceptible to our health warnings. Another important point is that we controlled the smoking cessation interventions in the experimental group and control group 1 to assess the "net" effect of confronting smokers with COPD, whereas Parkes et al. did not control the smoking treatments smokers used following confrontation with spirometry. It may be that these smokers made more use of, and were more compliant with evidence-based treatments for smoking cessation. All these differences in recruitment strategies are very important when interpreting the results; using spirometry in average (and mostly healthy) smokers who are interested in their lung function may trigger an attempt to quit smoking (and increase the likelihood of quitting), but in smokers with COPD who are interesting in quitting, confrontation with the results from smoking does not seem to be effective. Another point to be mentioned when comparing the results of our study with those of Parkes et al. is the limitation of the latter study to use a point prevalence estimate as primary outcome; the rate of non-smokers at 52 weeks. We used three follow-up measurements to calculate prolonged abstinence from smoking rates from week 5 through week 52, as recommended by the Society for Research on Nicotine and Tobacco. ${ }^{38}$ Furthermore, the average age of the participants from our study was comparable with the participants from the study of Parkes et al., but our participants had a heavier smoking history: 44 pack-years compared with 31 pack-years in the study of Parkes et al. This indicates higher levels of nicotine and tobacco addiction which is associated with a lower likelihood of smoking cessation.

Taking a closer look at the results from our study, it appears as if our confrontational counselling approach did have a short-term effect on smoking cessation; the abstinence rate at 5 weeks after the target quit date was almost $12 \%$ higher in the experimental group compared with control group $1(O R=1.60)$, which is a clinically relevant difference for a comparison of two equally intensive treatments. The $p$-value 
was only marginally significant $(p=0.08)$, but would probably have reached a level below 0.05 when the sample size (and therefore the power) would have been bigger. After adjusting for baseline covariates, the odds ratio increased to $O R=2.01$ with a $p$ value of 0.023 . These findings suggest that confrontation counselling was likely to have an effect until shortly after the completion of the counselling treatment but that this effect diminished during the follow-up period in which the participants did not receive any more counselling.

To the best of our knowledge, only two other trials studied the efficacy of antidepressants for smoking cessation in smokers with COPD. Tashkin et al. performed a randomized trial on the efficacy of bupropion for smoking cessation in 404 smokers with COPD. ${ }^{39}$ The abstinence rate in the bupropion group after 26 weeks follow-up was $16 \%$ (compared with $9 \%$ in the placebo group). Wagena et al. performed a randomized trial on the efficacy of bupropion and nortriptyline for smoking cessation in 255 smokers of which $56 \%$ had COPD. ${ }^{40}$ The abstinence rates after 26 weeks follow-up were $27 \%$ in the bupropion subgroup with COPD and $21 \%$ in the nortriptyline subgroup with COPD (compared with $8 \%$ in the placebo group). The subgroup of smokers with COPD that received nortriptyline had a lower abstinence rate than the subgroup of smokers with normal lung function (32\%), indicating a lower likelihood of quitting in smokers with airflow limitation. These results are comparable with the results from our study and underline the high relapse rates in smokers with COPD who try to quit smoking.

A major strength of the present study is that all factors which are known to be associated with abstinence from smoking were standardised in both the experimental group and control group 1: type of counsellor (respiratory nurse), type of counselling (face-to-face and by telephone), number and duration of counselling sessions, and type (nortriptyline) and dosage of smoking cessation medication. The baseline risk for COPD of all participants was the same; they all had previously undetected mild to moderate airflow limitation. Only participants from the experimental group were confronted with their disease, and therefore we are able to assess the "net" effect of confronting and counselling smokers with COPD. We were not able to find a statistical significant difference in abstinence rates between the experimental group and control group 2 from week 5 through 52. The sample size calculation was based on the assumption that a higher proportion of smokers would quit in the experimental group. The study was not sufficiently powered to detect the observed difference of $5 \%$.

We conclude that confronting smokers who are interested in quitting smoking with previously undetected COPD does not increase long-term smoking cessation rates. Confrontational counselling may have short-term effects, but these diminish during the first year after initial counselling treatment. The high failure rates dramatically 
emphasize the difficulty tobacco addicted smokers experience with quitting smoking and highlight the need for treating tobacco addiction as a chronic relapsing disorder and to match it with an appropriate and tailored amount of care. This is especially indicated in smokers with respiratory disease, who have a more urgent need to stop smoking. Future research should investigate whether repeated counselling sessions during a longer follow-up period can consolidate an initial short-term effect and therefore increase long-term smoking cessation rates. 
Table 1: Components of counselling in experimental group and control group 1

General components in both experimental group and control group

FC1 (day 1): 40 min. counselling by RN

- assess and discuss smoking characteristics

- assess and increase motivation to quit

- discuss cons of smoking and pros of quitting

- start use of nortriptyline

FC2 (day 8): 40 min. counselling by RN

- evaluate use nortriptyline

- assess and increase self-efficacy to quit

- prepare of the TQD

- anticipate on barriers of quitting and withdrawal

TQD: TC (day 14): 5 min. counselling by RN

- evaluate the quit attempt

- give advice about quitting and abstaining

FC3 (day 15): 40 min. counselling by RN

- evaluate quit attempt

- evaluate use nortriptyline

- give advice about relapse prevention

FC4 (day 22): 40 min. counselling by RN

- evaluate quit attempt

- evaluate use nortriptyline

- give advice about relapse prevention

- end counselling

Additional components of confrontational counselling in the experimental group only

Incorporated in $\mathrm{FC} 1+2$

- discuss the results from spirometry

- confront with the consequences of smoking: the diagnosis COPD

- discuss the severity and prognosis of COPD and the benefits of quitting smoking by using the "Fletcher curve" and images of normal and smoker's lungs ${ }^{33}$

Incorporated in $\mathrm{FC} 3+4$

- reflect on the smoker's thoughts, feelings, and beliefs about COPD

- challenge irrational beliefs about smoking by raising the smoker's consciousness about these beliefs, testing

their reality, and by exploring the relationship between beliefs and behaviour

- use of a smoking cessation diary to monitor smoking behaviour and beliefs about smoking

$\mathrm{FC}=$ face-to-face counselling session; $\mathrm{TC}=$ telephone counselling session; $\mathrm{TQD}=$ target quit date; $\mathrm{RN}=$ respiratory nurse. 


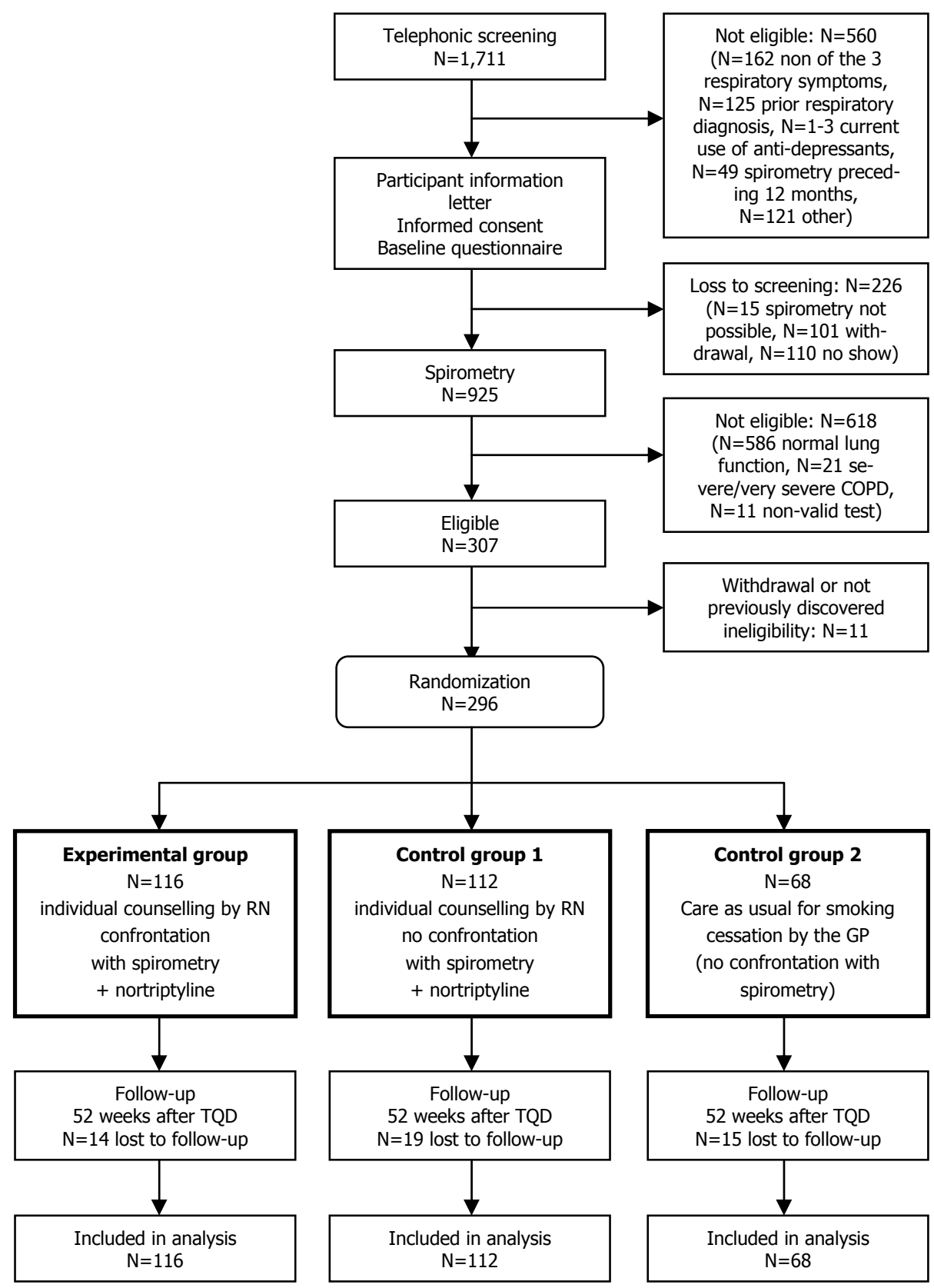

Figure 1: Study flowchart. TQD=target quit date; $R N=$ respiratory nurse; $G P=$ general practitioner 
Table 2: Baseline characteristics of participants

\begin{tabular}{|c|c|c|c|}
\hline Characteristic & $\begin{array}{l}\text { Experimental group } \\
(\mathrm{EX}) \\
\mathrm{N}=116\end{array}$ & $\begin{array}{c}\text { Control group } 1 \\
\text { (C1) } \\
N=112\end{array}$ & $\begin{array}{c}\text { Control group } 2 \\
\text { (C2) } \\
N=68\end{array}$ \\
\hline Years of age & $53.8(7.0)$ & $54.9(8.0)$ & $53.0(7.6)$ \\
\hline Male sex, N (\%) & $71(61.2)$ & $74(66.1)$ & $40(58.8)$ \\
\hline Level of educational background* & $3.9(1.7)$ & $3.8(1.8)$ & $4.0(1.9)$ \\
\hline Body Mass Index, $\mathrm{kg} / \mathrm{m}^{2}$ & $25.0(4.0)$ & $25.3(4.1)$ & $24.8(4.2)$ \\
\hline Cigarettes per day & $23.9(8.4)$ & $23.2(9.9)$ & $22.7(9.6)$ \\
\hline Pack years ${ }^{\dagger}$ & $44.1(18.3)$ & $44.2(19.1)$ & $41.5(19.7)$ \\
\hline Nicotine dependence, FTND $^{\ddagger}$ & $4.6(1.5)$ & $4.5(1.5)$ & $4.4(1.5)$ \\
\hline Number of previous quit attempts & $3.5(3.5)$ & $4.0(5.7)$ & $4.2(3.3)$ \\
\hline \multicolumn{4}{|l|}{ Previous use of pharmacotherapy for smoking } \\
\hline cessation, N (\%) & $30(25.9)$ & $36(32.1)$ & $30(44.1)$ \\
\hline nicotine gum & $60(51.7)$ & $51(45.5)$ & $35(51.5)$ \\
\hline $\begin{array}{l}\text { nicotine patch } \\
\text { bupropion }\end{array}$ & $23(19.8)$ & $23(20.5)$ & $18(26.4)$ \\
\hline $\begin{array}{l}\text { Previous use of individual counselling for } \\
\text { smoking cessation, } \mathrm{N}(\%)\end{array}$ & $7(6.0)$ & $4(3.6)$ & $5(7.3)$ \\
\hline Clinical control of COPD, $\mathrm{CCQ}^{\neq}$ & $1.3(0.7)$ & $1.3(0.8)$ & $1.2(0.8)$ \\
\hline \multicolumn{4}{|l|}{ Disease specific quality of life, CRQ-SR } \\
\hline fatigue & $4.7(1.1)$ & $4.8(1.2)$ & $4.6(1.4)$ \\
\hline emotional function & $4.8(1.1)$ & $4.8(1.2)$ & $4.8(1.2)$ \\
\hline mastery & $5.0(0.8)$ & $4.9(0.8)$ & $5.0(0.8)$ \\
\hline Anxiety, HADS ${ }^{\#}$ & $5.6(3.7)$ & $6.0(3.9)$ & $5.9(4.1)$ \\
\hline $\mathrm{FEV}_{1}$ post-bd. $\%$ pred. & $80.5(14.7)$ & $83.7(16.8)$ & $79.7(14.0)$ \\
\hline FVC post-bd. \% pred. & $103.9(14.9)$ & $107.6(17.8)$ & $105.4(14.4)$ \\
\hline $\begin{array}{l}\text { FEV } 1 / \text { FVC post-bd. } \\
\text { GOLD classification" }\end{array}$ & $62.5(5.9)$ & $63.0(6.1)$ & $61.9(6.3)$ \\
\hline GOLD 1 (mild COPD) & $62(53.4)$ & $66(58.9)$ & $32(47.1)$ \\
\hline GOLD 2 (moderate COPD) & $54(46.6)$ & $46(41.1)$ & $36(52.9)$ \\
\hline
\end{tabular}

Data are presented as mean (SD), unless otherwise stated. *Educational background: range from 1 (lowest education) through 7 (highest). ${ }^{\dagger} 1$ pack year $=$ number of cigarettes smoked per day $\mathrm{x}$ number of years smoking / 20. "Fagerström Test for Nicotine Dependence: range from 0 (lowest level of nicotine dependence) through 10 (highest). ${ }^{\neq}$Clinical COPD Questionnaire: range from 0 (very good control of COPD) through 6 (very poor). ${ }^{\ngtr}$ Chronic Respiratory Disease Questionnaire self-rated: range per sub domain from 1 (maximum impairment) through 7 (minimum). ${ }^{\#}$ Hospital Anxiety and Depression Scale: range from 0 (lowest degree of anxiety) through 21 (highest)]. "Global Initiative for Chronic Obstructive Lung Disease.

Table 3: Cotinine validated abstinence from smoking rates

\begin{tabular}{|c|c|c|c|c|c|c|}
\hline \multirow{4}{*}{$\begin{array}{l}\text { Weeks } \\
\text { from target } \\
\text { quit date } \\
5\end{array}$} & \multirow{3}{*}{$\begin{array}{c}\text { Experimental } \\
\text { group (EX) } \\
N(\%)\end{array}$} & \multirow{3}{*}{$\begin{array}{c}\text { Control } \\
\text { group } 1(\mathrm{C} 1) \\
\mathrm{N}(\%)\end{array}$} & \multirow{3}{*}{$\begin{array}{c}\text { Control } \\
\text { group } 2(\mathrm{C} 2) \\
\mathrm{N}(\%)\end{array}$} & \multicolumn{3}{|c|}{ Odds Ratio for abstinence (95\%CI) } \\
\hline & & & & & $\mathrm{p}$-value & \\
\hline & & & & EX vs. $\mathrm{C} 1$ & EX vs. C2 & $\mathrm{C} 1$ vs. $\mathrm{C} 2$ \\
\hline & 59 (50.9) & $44(39.3)$ & $12(17.6)$ & $\begin{array}{c}1.60(0.91,2.80) \\
p=0.080\end{array}$ & $\begin{array}{c}4.83(2.23,10.64) \\
p<0.001\end{array}$ & $\begin{array}{c}3.02(1.38,6.70) \\
p=0.003\end{array}$ \\
\hline-26 & $35(30.2)$ & $26(23.2)$ & $8(11.8)$ & $\begin{array}{c}1.43(0.76,2.69) \\
p=0.236\end{array}$ & $\begin{array}{c}3.24(1.32,8.20) \\
p=0.006\end{array}$ & $\begin{array}{c}2.27(0.90,5.87) \\
p=0.062\end{array}$ \\
\hline-52 & $13(11.2)$ & $13(11.6)$ & $4(5.9)$ & $\begin{array}{c}0.96(0.40,2.34) \\
p=0.961\end{array}$ & $\begin{array}{c}2.02(0.58,7.70) \\
p=0.236\end{array}$ & $\begin{array}{c}2.10(0.60,8.02) \\
p=0.211\end{array}$ \\
\hline
\end{tabular}




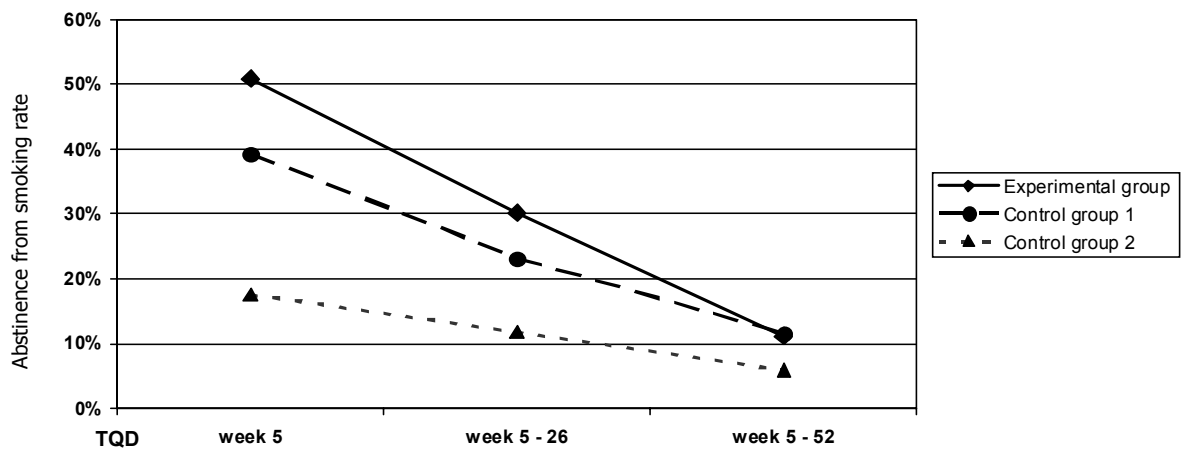

Figure 2: Cotinine validated abstinence from smoking rates during 52 weeks after the target quit date (TQD). 


\section{REFERENCES}

1 Global Initiative for Chronic Obstructive Lung Disease. Global strategy for the diagnosis, management, and prevention of chronic pulmonary disease. Executive summary (http://www.goldcopd.com): NHLBI / WHO, 2006

2 World Health Organisation (WHO). The World Health Report. Reducing risks, promoting healthy life. Geneva: WHO, 2002

3 Murray CJ, Lopez AD. Alternative projections of mortality and disability by cause 1990-2020: Global Burden of Disease Study. Lancet 1997; 349:1498-1504

4 Pauwels RA, Buist AS, Calverley PM, et al. Global strategy for the diagnosis, management, and prevention of chronic obstructive pulmonary disease. NHLBI/WHO Global Initiative for Chronic Obstructive Lung Disease (GOLD) Workshop summary. Am J Respir Crit Care Med 2001; 163:1256-1276

5 Anthonisen NR, Connett JE, Kiley JP, et al. Effects of smoking intervention and the use of an inhaled anticholinergic bronchodilator on the rate of decline of FEV1. The Lung Health Study. J Am Med Assoc 1994; 272:1497-1505

6 Pauwels PRA, Rabe KF. Burden and clinical features of chronic obstructive pulmonary disease (COPD). Lancet 2004; 364:613-620

7 van Schayck CP, van der Heijden FMMA, van den Boom G, et al. Underdiagnosis of asthma: is the doctor or the patient to blame? The DIMCA project. Thorax 2000; 55:562-565

8 Boushey H, Enright P, Samet J. Spirometry for Chronic Obstructive Pulmonary Disease Case Finding in Primary Care? Am J Respir Crit Care Med 2005; 172:1481-1482

9 Enright PL, Studnicka M, Zielinski J. Spirometry to detect and manage chronic obstructive pulmonary disease and asthma in the primary care setting. Eur Respir Mon 2005; 31:1-14

10 White P. Should we use spirometry in the early detection of COPD? Eur Respir J 2005; 26:558-559

11 Wilt TJ, Niewoehner D, Kane RL, et al. Spirometry as a motivational tool to improve smoking cessation rates: a systematic review of the literature. Nicotine Tob Res 2007; 9:21-32

12 Wilt TJ, Niewoehner D, Kim C-B, et al. Use of Spirometry for case finding, diagnosis, and management of chronic obstructive pulmonary disease (COPD). Evidence Report/Technology Assessment No. 121 (Prepared by the Minnesota Evidence-based Practice Center under Contract No. 290-02-0009.) AHRQ Publication No. 05-E017-2. Rockville, MD.: Agency for Healthcare Research and Quality, 2005

13 Enright P. Does screening for COPD by primary care physicians have the potential to cause more harm than good? Chest 2006; 129:833-835

14 McBride CM, Emmons KM, Lipkus IM. Understanding the potential of teachable moments: the case of smoking cessation. Health Educ Res 2003; 18:156-170

15 Aveyard P, Brown K, Saunders C, et al. Weekly versus basic smoking cessation support in primary care: a randomised controlled trial. Thorax 2007; 62:898-903

16 Parkes G, Greenhalgh T, Griffin M, et al. Effect on smoking quit rate of telling patients their lung age: the Step2quit randomised controlled trial. BMJ 2008; 336:598-600

17 Kotz D, Huibers MJ, Vos R, et al. Principles of confrontational counselling in smokers with chronic obstructive pulmonary disease (COPD). Med Hypotheses 2008; 70:384-386

18 Kotz D, Wesseling GJ, Huibers MJH, et al. Efficacy of confrontational counselling for smoking cessation in smokers with previously undiagnosed mild to moderate airflow limitation: study protocol of a randomized controlled trial. BMC Public Health 2007; 7:332

19 Zelen M. A new design for randomized clinical trials. N Engl J Med 1979; 300:1242-1245

20 Zelen M. Randomized consent designs for clinical trials: an update. Stat Med 1990; 9:645-656

21 Torgerson DJ, Roland M. What is Zelen's design? BMJ 1998; 316:606

22 Miller MR, Hankinson J, Brusasco V, et al. Standardisation of spirometry. Eur Respir J 2005; 26:319-338

23 Miller MR, Crapo R, Hankinson J, et al. General considerations for lung function testing. Eur Respir J 2005; 26:153-161

24 STIVORO. De L-MIS: stoppen met roken voor longpatiënten. Handleiding voor longverpleegkundigen. [The L-MIS: smoking cessation in lung patients. Strategy for respiratory nurses]. Den Haag: STIVORO, 2003 25 Hughes JR, Stead LF, Lancaster T. Antidepressants for smoking cessation. Cochrane Database Syst Rev 2004 
26 Wagena EJ, Knipschild P, Zeegers MPA. Should nortriptyline be used as a first-line aid to help smokers quit? Results from a systematic review and meta-analysis. Addiction 2005; 100:317-326

27 Pieterse ME, Seydel ER, DeVries $H$, et al. Effectiveness of a minimal contact smoking cessation program for Dutch general practitioners: a randomized controlled trial. Prev Med 2001; 32:182-190

28 SRNT Subcommittee on Biochemical Verification. Biochemical verification of tobacco use and cessation. Nicotine Tob Res 2002; 4:149-159

29 Heatherton TF, Kozlowski LT, Frecker RC, et al. The Fagerström Test for Nicotine Dependence: a revision of the Fagerström Tolerance Questionnaire. Br J Addict 1991; 86:1119-1127

30 van der Molen T, Willemse BWM, Schokker S, et al. Development, validity and responsiveness of the Clinical COPD Questionnaire. Health and Quality of Life Outcomes 2003; 1

31 van der Molen T, Willemse BW, Schokker S, et al. Development \& cross sectional validity of the COPD symptom control questionnaire. Prim Care Resp J 2001; 10:75

32 Williams JEA, Singh SJ, Sewell L, et al. Development of a self-reported Chronic Respiratory Questionnaire (CRQ-SR). Thorax 2001; 56:954-959

33 Guyatt GH, Berman LB, Townsend M, et al. A measure of quality of life for clinical trials in chronic lung disease. Thorax 1987; 42:773-778

34 Caponnetto P, Polosa R. Common predictors of smoking cessation in clinical practice. Respir Med 2008

35 Zigmond AS, Snaith RP. The Hospital Anxiety and Depression Scale. Acta Psyciatr Scan 1983; 67:361-370

36 Bednarek M, Gorecka D, Wielgomas J, et al. Smokers with airway obstruction are more likely to quit smoking. Thorax 2006; 61:869-873

37 Kotz D, van Schayck CP, Huibers MJ, et al. Assessing the efficacy of spirometry for smoking cessation. Thorax 2007; 62:742

38 Hughes J, Keely J, Niaura R, et al. Measures of abstinence in clinical trials: issues and recommendations. Nicotine \& Tobacco Research 2003; 5:13-26

39 Tashkin D, Kanner R, Bailey W, et al. Smoking cessation in patients with chronic obstructive pulmonary disease: a double-blind, placebo-controlled, randomised trial. Lancet 2001; 357:1571-1575

40 Wagena EJ, Knipschild PG, Huibers MJH, et al. Efficacy of Bupropion and Nortriptyline for Smoking Cessation Among People at Risk for or With Chronic Obstructive Pulmonary Disease. Arch Intern Med 2005; 165:2286-2292

41 Fletcher C, Peto R. The natural history of chronic airflow obstruction. Brit Med J 1977; 1:1645-1648 



\title{
Chapter 8
}

\section{What mediates the effect of confrontational counselling on smoking cessation in smokers with COPD?}

\author{
Daniel Kotz ${ }^{1}$ \\ Marcus J.H. Huibers ${ }^{2}$ \\ Robert J. West ${ }^{3}$ \\ Geertjan Wesseling ${ }^{4}$ \\ Onno C.P. van Schayck ${ }^{1}$
}

\section{Submitted for publication}

\footnotetext{
${ }^{1}$ Department of General Practice, School for Public Health and Primary Care (CAPHRI), Maastricht University Medical Centre, Maastricht, the Netherlands. ${ }^{2}$ Department of Clinical Psychological Science, Maastricht University, Maastricht, the Netherlands. ${ }^{3}$ Cancer Research UK Health Behaviour Unit, Department of Epidemiology and Public Health, University College London, London, UK. ${ }^{4}$ Department of Respiratory Medicine, Maastricht University Medical Centre, Maastricht, the Netherlands.
} 


\section{ABSTRACT}

\section{Objective}

Within the framework of a randomized, active treatment controlled trial, we used a mediation analysis to understand the mechanisms by which an intervention that uses confrontation with spirometry for smoking cessation achieves its effects.

\section{Methods}

Participants were 228 smokers from the general population with previously undetected chronic obstructive pulmonary disease (COPD), who were detected with airflow limitation by means of spirometry. They received two equally intensive behavioural treatments by a respiratory nurse combined with nortriptyline for smoking cessation: confrontational counselling with spirometry versus conventional health education and promotion (excluding confrontation with spirometry and COPD).

\section{Results}

Cotinine validated abstinence rates from smoking at 5 weeks after the target quit date were $43.1 \%$ in the confrontational counselling group versus $31.3 \%$ in the control group $(\mathrm{OR}=1.67,95 \% \mathrm{CI}=0.97-2.87)$. The effect of confrontational counselling on abstinence was independently mediated by the expectation of getting a serious smoking related disease in the future $(\mathrm{OR}=1.76,95 \% \mathrm{CI}=1.03-3.00)$, selfexempting beliefs $(\mathrm{OR}=0.42,95 \% \mathrm{CI}=0.21-0.84)$, and self-efficacy $(\mathrm{OR}=1.38$, $95 \% \mathrm{CI}=1.11-1.73)$.

\section{Conclusion}

This study is the first to attempt a mediation analysis of the treatment effects of confronting smokers with the results from spirometry and the diagnosis COPD. The study design accounts for potential biases and enables us to isolate the effects of labelling with disease and to unseal the "black box" of mechanisms of change which may account for the effects of the intervention. We conclude that confrontational counselling increases risk perceptions and self-efficacy, and decreases self-exempting beliefs (risk denial) in smokers with previously undetected COPD. These changes in mediators are associated with a higher likelihood of smoking cessation.

\section{Practice implications}

Combining counselling with pharmacotherapy may increase the chances of smokers to quit smoking. Apart from the intensity, the content of counselling may be an important factor of success. A confrontational counselling approach as we applied may have the potential to alter smoking-related cognitions in such a way that smokers are more successful in quitting smoking. Nurses can be trained to deliver this treatment. 


\section{INTRODUCTION}

Chronic obstructive pulmonary disease (COPD) is a preventable and treatable disease which is characterized by airflow limitation that is not fully reversible. ${ }^{1}$ Spirometry is the gold standard for the diagnosis and assessment of the disease. ${ }^{1}$ COPD is currently the fifth leading cause of death worldwide ${ }^{2}$, and projections for 2020 indicate further increase in global mortality, placing COPD on the third position of lethal diseases. ${ }^{3}$ Cigarette smoking is by far the most important risk factor for COPD, and smoking cessation is the single most effective way to reduce the risk of developing COPD and to affect the outcome in patients at all stages of the disease., ${ }^{4,5}$

Discussing abnormal test results with smokers has been suggested as a "teachable moment" that may increase motivation to quit smoking, but there is only weak evidence to support such an approach. ${ }^{6}$ Various studies have been performed on the efficacy of spirometry as a motivational tool for smoking cessation but their results are inconclusive ${ }^{7-9}$ Findings are often of limited validity because of one or more important biases such as unstandardized counselling intensity, incomparable or uncontrolled use of pharmacological aids for smoking cessation between experimental and control group, or different (or unclear) baseline levels of lung function and motivation to quit smoking. ${ }^{10} \mathrm{~A}$ recent randomized trial clearly showed a positive effect; telling smokers their "lung age" (based on spirometry) increased the 12month abstinence rate by $7.2 \%$, but the mechanisms by which the intervention achieved its effect were unclear. ${ }^{11}$

We conducted a randomized controlled trial on the efficacy of what we have termed "confrontational counselling"12. This is a patient-centred approach which involves confronting smokers with the consequences of their addiction (previously undiagnosed COPD) and which uses specific communication skills to identify and challenge irrational beliefs about smoking. In this article, we will use a mediation analysis to understand how exactly confrontational counselling may effect short-term abstinence from smoking (five weeks after the target quit date). We hypothesised that confrontational counselling - through labelling with a potentially life-threatening illness status - increases risk perception and health concerns and decreases self-exempting beliefs in smokers, factors which in turn account for abstinence from smoking (see figure 1). Mediation analysis allows us to open up the "black box" that conceals the mechanisms of change in our intervention.

\section{METHODS}

We used data from a randomized controlled trial comparing two active smoking cessation treatments in smokers with previously undiagnosed COPD: medium intensi- 
ty confrontational counselling delivered by a respiratory nurse combined with nortriptyline for smoking cessation (experimental group) with medium intensity health education and promotion delivered by a respiratory nurse combined with nortriptyline for smoking cessation (control group). The third trial arm, "care as usual by the GP", was not included in the current analysis because the goal was to assess mediation of the specific effect of the nurses' counselling. The trial has been approved by the medical ethics committee of Maastricht University and Maastricht University Hospital and registered at the Netherlands Trial Register (ISRCTN 64481813). A detailed description of the trial protocol has been published elsewhere. ${ }^{13}$

\section{Recruitment, eligibility, informed consent, and randomization of participants}

Current smokers aged 35 through 70 years who were interested in quitting smoking were recruited from the general population and from primary care practices in Dutchand Belgian-Limburg (the region surrounding Maastricht). They were invited to take part in a study on individual counselling and medication for smoking cessation. Neither information about the target condition we were looking for (COPD) nor the difference in individual counselling between the groups was given to participants before randomization.

Eligibility was assessed during an initial telephone interview. Inclusion criteria were: smoking history of 10 or more pack years (= number of cigarettes smoked per day $\mathrm{x}$ number of years smoking / 20); being competent to read and speak Dutch; and reporting at least one of the respiratory symptoms: cough, sputum production, or shortness of breath. Exclusion criteria were: evidence of a prior respiratory diagnosis, defined by an affirmative answer to the question "Do you have COPD, chronic bronchitis, asthma or asthmatic bronchitis?". Subjects were also not allowed to have undergone a lung function test (spirometry) during the preceding 12 months. One or more contraindications for using the smoking cessation medication (nortriptyline) were also criteria for exclusion, among others the current use of anti-depressants.

Subjects filled out a baseline questionnaire at home and handed it in during the spirometry visit. Spirometry was performed according to American Thoracic Society (ATS) / European Respiratory Society (ERS) criteria ${ }^{14,15}$ using a Vitalograph ${ }^{\circledR} 2120$ (Vitalograph Ltd, Buckingham, England). Subjects were eligible if they had airflow limitation defined as post-bronchodilator Forced Expiratory Volume in one second $\left(\mathrm{FEV}_{1}\right)$ / Forced Vital Capacity (FVC) $<70 \%$ in combination with post-bronchodilator $\mathrm{FEV}_{1} \geq 50 \%$ of predicted value; i.e. mild or moderate airflow limitation, according to the international GOLD guideline ${ }^{1}$. The results of spirometry were not discussed at that moment. Eligible subjects were contacted by telephone shortly after spirometry and randomized using a database which precluded that any person involved in the 
study could predict or influence which treatment group the next participant would be allocated to.

\section{Interventions}

Participants from both the experimental group and the control group received medium intensity counselling delivered by a respiratory nurse combined with nortriptyline for smoking cessation. The common basis for the counselling in both groups was the so-called "L-MIS" protocol for the treatment of nicotine and tobacco addiction which had been implemented among all respiratory nurses in the Netherlands in previous years (table 1, top). ${ }^{16}$ Specific elements of "confrontational counselling" ${ }^{12}$ were added to the L-MIS in the experimental group which discriminated the treatment from that in the control group (table 1, bottom). Confrontational counselling is a patient-centred approach which involves communication skills and elements of cognitive therapy. ${ }^{12,13}$ It aims to identify certain cognitions about smoking such as health concerns, risk perception, and self-exempting beliefs. The beliefs were challenged by the respiratory nurse during the counselling sessions. Notes from a smoking cessation diary that smokers from the experimental group used served as input for the counselling.

Participants from both groups received an equal dosage of nortriptyline (Nortrilen ${ }^{\circledR}$ ) for smoking cessation. Nortriptyline is a tricyclic anti-depressant which has been shown to be a cheap and effective alternative for the anti-depressant bupropion $\left(Z y b a{ }^{\mathrm{TM}}\right) .{ }^{17,18}$ The nurse monitored the correct use of the medication and the occurrence of side-effects. In case of unpleasant or severe side-effects, the dosage was reduced or the use of the medication was stopped.

\section{Measurements}

Participants completed a questionnaire at baseline and at follow-up (day 50; approximately 5 weeks after the quit date). Health perceptions included self-designed questions measuring the domains health concerns ( 3 single items), risk perception (2 single items), and self-exempting beliefs on a 5-point Likert scale (for an overview of these questions see the appendix). Self-exempting beliefs are statements of risk denial that smokers may use to relieve fear and anxiety that may be associated with the detrimental consequences of smoking on health. The 9 items measuring these beliefs were combined into one scale which showed a good reliability (Cronbach's $a=0.84$ ). Quality of life was measured with the Short-form 36-item questionnaire $(\mathrm{SF}-36)^{19,20}$, the Chronic Respiratory Questionnaire self-reported (CRQ) ${ }^{21,22}$, and the EuroQol (EQ-5D) $)^{23,24}$. Mental health (depression and anxiety) was measured with the Beck Depression Inventory $(\mathrm{BDI})^{25}$ and the Hospital Anxiety and Depression Scale $(\mathrm{HADS})^{26-28}$. 
Motivation to quit was measured by the nurse during FC1 by asking: "How important do you find it to stop smoking on a scale from 0 (not important at all) through 10 (very important)?". Self-efficacy towards smoking cessation was measured by the nurse during FC2, thus before the target quit date, by asking: "How high do you consider your chance to succeed in stopping smoking on a scale from 0 (very low) through 10 (very high)?".

To validate non-smoking, urine was collected from every self-reported non-smoker at the follow-up visit and analysed at the laboratory of the Department of Health Risk Analysis and Toxicology (GRAT) of Maastricht University. The concentration of cotinine in urine was measured by a highly specific radioimmunoassay using monoclonal antibodies. ${ }^{29}$

The outcome measure for the current analysis was abstinence from smoking at 5 weeks after the target quit date. A participant was defined as abstinent from smoking if both of the following two conditions were met: urine cotinine level of $<50 \mathrm{ng} / \mathrm{mL}^{30}$ in combination with self-reported non smoking (i.e. not a single cigarette since the target quit date). All randomized subjects were included in an intention-to-treat analysis and subjects not showing up at the follow-up visit or with a missing value on one of the two criteria for abstinence were regarded as smokers.

\section{Statistical analyses}

Baseline demographic and smoking characteristics as well as mean scores of health perceptions, quality of life, and mental health were compared to assess potential differences between the groups before treatment. Within-subject difference scores were calculated by subtracting the score at baseline from the score at follow-up (except for self-efficacy which was measured at one point of time). Statistical differences in mean-difference scores between the groups were tested with t-tests. Candidate variables $(p<0.05)$ were entered into the mediation analysis.

We used an approach for the mediation analysis which was based on a method described by Baron \& Kenny. ${ }^{31}$ They define a mediator as a variable that "accounts for the relation between the predictor and the criterion". In our case, the mediator would account for the relation between intervention and abstinence from smoking. The mediation analysis comprised four steps for each candidate mediator using regression analysis. In step 1 , we regressed abstinence (1=abstinent from smoking, $0=$ not abstinent) on intervention ( 1 =experimental group, $0=$ control group) in a simple logistic regression. In step 2, we regressed the candidate mediator on intervention in a simple linear regression. In step 3, we regressed abstinence on the candidate mediator, again in a simple logistic regression. In step 4, we regressed abstinence on intervention and the candidate mediator in a multiple logistic regres- 
sion model. We defined a mediator as a variable that was significantly (at $p<0.1$ ) associated with the intervention in step 2 and with the outcome in steps 3 and 4.

Finally, we built multiple logistic regression models with all mediators verified by the previous mediation analysis. In model 1 , we regressed abstinence on intervention. In model 2, we added all verified mediators. We then adjusted model 2 for all important baseline characteristics.

We did not impute missing values. The percentage missing values on the measures of health perceptions, quality of life, and mental health was on average $14 \%$ in the experimental group and $15 \%$ in the control group. In ancillary analyses, we ran all logistic regression models with missing values imputed by the series mean.

\section{RESULTS}

A total of 116 smokers with previously undetected COPD were randomly allocated to the experimental group, 112 to the control group. The baseline characteristics are shown in table 2. Mean baseline scores on all measures of health perceptions, quality of life, and mental health were comparable between the groups (not shown in the table), except for the subscale "physical role limitations" on the SF-36; the mean score in the experimental group was substantially higher $(M=84.1, S D=30.0)$ than in the control group $(M=73.4,39.1)$. The abstinence from smoking 5 weeks after the target quit date were $43.1 \%(\mathrm{~N}=50$ of 116$)$ in the experimental group and $31.3 \%$ $(\mathrm{N}=35$ of 112$)$ in the control group ( $\mathrm{OR}=1.67,95 \% \mathrm{CI}=0.97-2.87, \mathrm{p}=0.065,2$-tailed). This association remained unchanged when adjusting for the variable "physical role limitations" on the SF-36.

\section{Effect of the interventions on health perceptions, quality of life, mental health, and self-efficacy}

Table 3 shows the mean-difference scores between baseline and follow-up for the experimental group and the control group, and the difference in means between the groups (experimental group minus control group). A positive mean-difference indicates an increase of the measure from baseline to follow-up in the experimental group relative to the control group, and a negative mean-difference a relative decrease in the experimental group. Four measures of health perceptions were significantly increased in the experimental group: health concerns 1 (meandifference $=0.81)$, health concerns $3(0.35)$, risk perception $1(0.32)$, and risk perception 2 (0.34). A fifth measure, self-exempting beliefs, was significantly decreased in the experimental group (-0.31). There were no differences between the groups with regard to measures of quality of life and mental health. Self-efficacy towards smoking cessation, which was measured at one point in time (at $\mathrm{FC} 2$, before the quit 
date), and is therefore not shown in table 3, was significantly higher in the experimental group $(M=8.4, S D=2.0)$ than in the control group $(M=7.7, S D=1.7$; $\mathrm{p}=0.004)$.

\section{Mediators of the association between intervention and abstinence}

The five measures of health perceptions and the variable self-efficacy were entered into the mediation/moderation analysis. In step 1, the intervention was associated with abstinence from smoking: the odds of abstinence was $67 \%$ higher in the experimental group than in the control group $(\mathrm{OR}=1.67,95 \% \mathrm{CI}=0.97-2.87, \mathrm{p}=0.065)$. Three measures were verified as mediators of the association between intervention and abstinence in steps 2 through 4: risk perception 2, self-exempting beliefs, and self-efficacy. In step 4, the odds for being abstinent from smoking adjusted for the effects of variable intervention per one point increase on the scale of the mediator were: $\mathrm{OR}=1.40(95 \% \mathrm{CI}=0.96-2.04, \mathrm{p}=0.081)$ for risk perception 2 , $O R=0.58$ (95\%CI $=0.34-1.01, \quad \mathrm{p}=0.052)$ for self-exempting beliefs, and $\mathrm{OR}=1.23$ $(95 \% \mathrm{CI}=1.03-1.46, \mathrm{p}=0.020)$ for self-efficacy.

Running all models with missing values replaced by the series mean resulted in similar results except for the variable risk perception 2 that failed to be a mediator.

We also performed a moderation analysis ${ }^{31}$ but did not find a variable that affected the direction and/or strength of the relation between intervention and abstinence.

\section{Adjusted effects of mediators of the association between intervention and abstinence}

The three verified mediators were entered into a multiple logistic regression, modelling abstinence from smoking (table 4). When the mediators were entered into model 2 , the effect of the intervention was eliminated $(O R=0.87, p=0.687)$. This does not mean that the intervention was ineffective, but rather that "perfect mediation"31 occurred: risk perception, self-exempting beliefs, and self-efficacy accounted for the effect of confrontational counselling on abstinence from smoking. The mediators had an independent effect on abstinence after adjustment for the potentially confounding baseline characteristics: age, sex, educational background, nicotine dependence, motivation to quit, number of previous quit attempts, airflow limitation, the subscale "physical role limitations" of the SF-36, and the other mediators. One point increase on the scale for risk perceptions 2 increased the odds of smoking abstinence by $76 \%$, and one point increase on the scale for self-efficacy increased the odds by $38 \%$. One point increase on the scale for self-exempting beliefs decreased the odds of smoking abstinence by $58 \%$.

When excluding the variable risk perception 2 from the adjusted model, the odds of smoking abstinence increased by $38 \%$ for one point increase on the scale for self- 
efficacy and decreased by $63 \%$ for one point increase on the scale for self-exempting beliefs.

\section{DISCUSSION}

We found three factors that mediated the effect of confrontational counselling on smoking cessation: self-efficacy towards successful smoking cessation, the expectation of getting a serious smoking-related disease within the next 10 years, and selfexempting beliefs towards smoking. All three mediators were independently associated with abstinence and accounted for the effect of the intervention.

The current evidence on the efficacy of spirometry as a motivational tool for smoking cessation is inconclusive. ${ }^{7,8}$ In one primary care study, for example, current smokers motivated to quit were randomized to undergo spirometry followed by brief advice for smoking cessation or to brief advice alone. ${ }^{32}$ The proportion of non-smokers was about 5\% higher in the spirometry group at 6, 12, and 24 months follow-up (not statistically significant at $p=0.05$ ). The study had several limitations including lack of information of actual use of smoking cessation medication in participants as well as lack of a valid measure of abstinence that meets international guidelines. ${ }^{30,33} \mathrm{~A}$ different and more valid primary care study clearly showed that telling smokers their "lung age" (based on spirometry) increased the 12-month abstinence rate by $7.2 \%$. However, the mechanisms by which the intervention achieved its effect were unclear. ${ }^{11}$ The present study may explain some of the underlying mechanisms.

In contrast to previous studies, our study is the first to attempt a mediation analysis of the treatment effects of confronting smokers with the results from spirometry and the diagnosis COPD. The design of our study accounts for potential biases by standardizing counselling intensity, using pharmacological aids for smoking cessation, and by randomizing smokers into two groups with comparable baseline levels of lung function and motivation to quit smoking. This design enables us to isolate the effects of labelling with disease and to unseal the "black box" of mechanisms of change which may account for the effects of the intervention.

There is sufficient evidence from the field of health education and promotion that fear arousal on its own is not a good motivator but that a combination of factors is more likely to result in change of health behaviour: high levels of perceived severity, susceptibility, outcome expectancy, and self-efficacy. ${ }^{34-36}$ Our experimental intervention, confrontational counselling, aimed to target these factors. The severity of the disease COPD was stressed by discussing the prognosis in case of continued smoking (deterioration of lung function resulting in decreased quality of life and finally premature death). The smoker's susceptibility was demonstrated by confronting the smoker 
with the results from spirometry and his/her respiratory symptoms. Our results show that confrontational counselling increased risk perception, which subsequently led to smoking cessation. Outcome expectancy and self-efficacy were triggered by making the smoker understand that there is an effective and feasible therapy for the disease: smoking cessation, aided by behavioural counselling and smoking cessation medication. Our results show that confrontational counselling increased self-efficacy, which again led to smoking cessation.

Self-exempting beliefs are common among smokers and these beliefs may interfere with smoking cessation. ${ }^{37-42}$ Smokers have these beliefs in order to reduce cognitive dissonance and alleviate anxiety. ${ }^{43}$ We challenged these beliefs by confronting smokers with objective evidence of the health consequences of their own smoking behaviour (COPD measured by spirometry) and by using specific communication skills deriving from cognitive therapy. The results from the mediation analysis show that confrontational counselling decreased self-exempting beliefs and that this led to smoking cessation.

The $p$-value resulting from statistical testing of the difference in observed effect between the intervention groups was $p=0.064$. A $p$-value is often misinterpreted as an indicator of clinical significance and misused when treated in a dichotomous manner as either "significant" $(p<0.05)$ or "not significant" $(p>0.05) .{ }^{44-46}$ The $p$-value does not provide information about the magnitude of the effect and the precision of the estimate, whereas confidence intervals do. ${ }^{44,47,48}$ The estimate of the effect size $(\mathrm{OR}=1.67)$ and the $95 \%$ confidence interval around this estimate $(0.97-2.87)$ show that the true population estimate is likely to be higher than 1 . Thus, the p-value would probably have reached a level below 0.05 when the sample size (and therefore the power) would have been bigger. It should also be noted that the observed effect size is large for a trial comparing two active treatments for smoking cessation which differ only in type of counselling and of clinical importance $(67 \%$ increase of the odds of abstinence from smoking in the experimental group compared with the control group). It should also be noted that the question if confrontational counselling is effective will be determined in a different analysis, as the efficacy of smoking cessation interventions should be based on the 12 month prolonged abstinence rates. $^{33}$ This mediation analysis concerns a different topic; understanding how confrontational counselling works.

The ancillary analyses with missing values replaced by the series mean resulted in similar results. However, the variable risk perception 2 failed to be a significant mediator. This may be an indication that this factor is a less strong mediator compared with the mediators self-exempting beliefs and self-efficacy. Removing the variable risk perception 2 from the final model only slightly changed the estimated odds ratios of the other two mediators, indicating the robustness of the model. 
Respiratory nurses from the experimental group received a group training and regular supervision in confrontational counselling. The effects of the intervention may be partly due to increased non-specific counselling skills of these nurses compared to nurses in the control group. One might therefore ascribe the combination of specific skills in confrontational counselling with training effects to the efficacy of the intervention.

The informed consent design we used raises some ethical issues as participants were not fully informed about the real purpose of the study, which is to detect and confront smokers with COPD. Participants randomized to the control group were not informed about their results of spirometry during the intervention but only after completing the follow-up period. This procedure was approved by a medical ethics committee and was regarded as ethical because smokers participating in this trial would probably not have been diagnosed outside the trial setting early due to the problem of underdiagnosis of COPD in primary care. The second reason is that all smokers from this trial received the most effective therapy for mild to moderate COPD, which is smoking cessation treatment.

Another important issue in the discussion about early detection of airflow limitation in smokers is the suggested counterproductive effect of communicating negative tests results (i.e. normal lung function) to smokers. ${ }^{49}$ Smokers with normal lung function might use their result from spirometry as a message that they are not susceptible to the effects of smoking and as an argument to continue smoking. If such an effect occurs, the abstinence rates of smokers diagnosed with normal lung function should be very low. In a recent large-scaled prospective study on the association between airway obstruction and smoking cessation in Poland, 12-month abstinence rates from smoking were only slightly lower in subjects with normal lung function (12\%) than in subjects with abnormal lung function. ${ }^{50}$ To test whether a counterproductive effect occurred in our sample, we randomized another 59 smokers with normal lung function (formally defined as GOLD 0 COPD) to the three intervention groups. Among these, 22 subjects (37\%) were abstinent from smoking at follow-up. This is about the same success rate as within the subgroup with GOLD 1 COPD (36\%; N=46/128) and within the subgroup with GOLD 2 COPD (39\%; $N=61 / 100)$. These findings do not provide evidence of counterproductive effects of reporting normal lung function in our sample. This might be due to the high initial motivation of all trial participants to stop smoking. This might also be due to the case-finding approach we chose. Therefore, our results may not be transferable to a large-scaled screening approach.

\section{Conclusion}

We conclude that confrontational counselling increases risk perceptions and selfefficacy, and decreases self-exempting beliefs in smokers with previously undetected 
COPD. These changes in mediators are associated with a higher likelihood of smoking cessation.

\section{Practice implications}

The best treatment for smoking cessation is a combination of evidence-based pharmacotherapy in combination with counselling. But with the currently available treatments, the majority of smokers fails to quit or relapses into smoking. It is therefore important to optimize available treatments and to develop new ones. The doseresponse relationship between counselling intensity and treatment success has been well established. In order to further increase the efficacy of counselling we must understand the psycho-social mechanisms that underlie changes in smoking behaviour and how these can be targeted. The results form our study show that risk perceptions, self-exempting beliefs, and self-efficacy are three important cognitions that may be associated with treatment success. Our confrontation counselling approach, delivered by trained respiratory nurses, altered these cognitions in such a way that smokers were more successful in quitting smoking in the short term. 


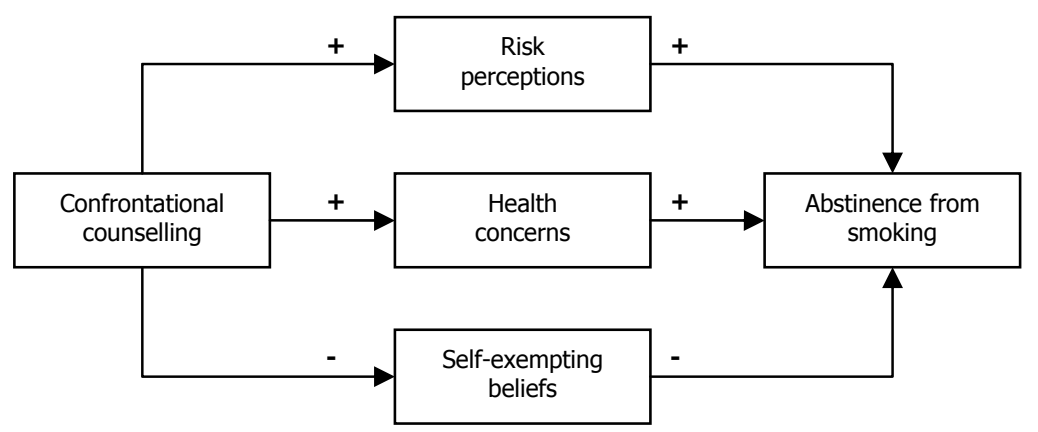

Figure 1: Hypothetical model of the mediating effects of confrontational counselling. 
Table 1: Components of counselling in experimental group and control group General components in both experimental group and control group

FC1 (day 1): 40 min. counselling by RN

- assess and discuss smoking characteristics

- assess and increase motivation to quit

- discuss cons of smoking and pros of quitting

- start use of nortriptyline

FC2 (day 8): 40 min. counselling by RN

- evaluate use nortriptyline

- assess and increase self-efficacy to quit

- prepare of the TQD

- anticipate on barriers of quitting and withdrawal

TQD: TC (day 14): 5 min. counselling by RN

- evaluate the quit attempt

- give advice about quitting and abstaining

FC3 (day 15): 40 min. counselling by RN

- evaluate quit attempt

- evaluate use nortriptyline

- give advice about relapse prevention

FC4 (day 22): 40 min. counselling by RN

- evaluate quit attempt

- evaluate use nortriptyline

- give advice about relapse prevention

- end counselling

Additional components of confrontational counselling in the experimental group only

Incorporated in $\mathrm{FC} 1+2$

- discuss the results from spirometry

- confront with the consequences of smoking: the diagnosis COPD

- discuss the severity and prognosis of COPD and the benefits of quitting smoking by using the "Fletcher curve" and images of normal and smoker's lungs ${ }^{58}$

Incorporated in $\mathrm{FC} 3+4$

- reflect on the smoker's thoughts, feelings, and beliefs about COPD

- challenge irrational beliefs about smoking by raising the smoker's consciousness about these beliefs, testing

their reality, and by exploring the relationship between beliefs and behaviour

- use of a smoking cessation diary to monitor smoking behaviour and beliefs about smoking

$\mathrm{FC}=$ face-to-face counselling session; $\mathrm{TC}=$ telephone counselling session; $\mathrm{TQD}=$ target quit date; $\mathrm{RN}=$ respiratory nurse.

Table 2: Demographic and smoking characteristics and lung function at baseline

\begin{tabular}{|c|c|c|}
\hline & $\begin{array}{l}\text { Experimental group } \\
\qquad \mathrm{N}=116\end{array}$ & $\begin{array}{l}\text { Control group } \\
\mathrm{N}=112\end{array}$ \\
\hline Years of age, mean (SD) & $53.8(7.0)$ & $54.9(8.0)$ \\
\hline Male sex, N (\%) & $71(61.2)$ & $74(66.1)$ \\
\hline $\begin{array}{l}\text { Educational background, median (range) [range from } 1 \\
\text { (lowest category) through } 7 \text { (highest)] }\end{array}$ & $4(1-7)$ & $4(1-7)$ \\
\hline Pack years ${ }^{\dagger}$, mean (SD) & $44.1(18.3)$ & $44.2(19.1)$ \\
\hline $\begin{array}{l}\text { FTND }^{\ddagger} \text {, mean (SD) [range from } 0 \text { (lowest level of nicotine } \\
\text { dependence) through } 10 \text { (highest)] }\end{array}$ & $4.6(1.5)$ & $4.5(1.5)$ \\
\hline $\begin{array}{l}\text { Motivation to quit: "How important do you find it to stop } \\
\text { smoking?", mean (SD) [range from } 0 \text { (not important at all) } \\
\text { through } 10 \text { (very important). }\end{array}$ & $9.0(1.2)$ & $8.8(1.2)$ \\
\hline Number of previous quit attempts, median (range) & $3(1-25)$ & $3(1-50)$ \\
\hline FEV $_{1}$ post-bd. \% pred. $^{*}$, mean (SD) & $80.5(14.7)$ & $83.7(16.8)$ \\
\hline FVC post- bd. \%pred. ${ }^{*}$, mean (SD) & $103.9(14.9)$ & $107.6(17.8)$ \\
\hline $\mathrm{FEV}_{1} / \mathrm{FVC}$ post-bd., mean (SD) & $62.5(5.9)$ & $63.0(6.1)$ \\
\hline
\end{tabular}




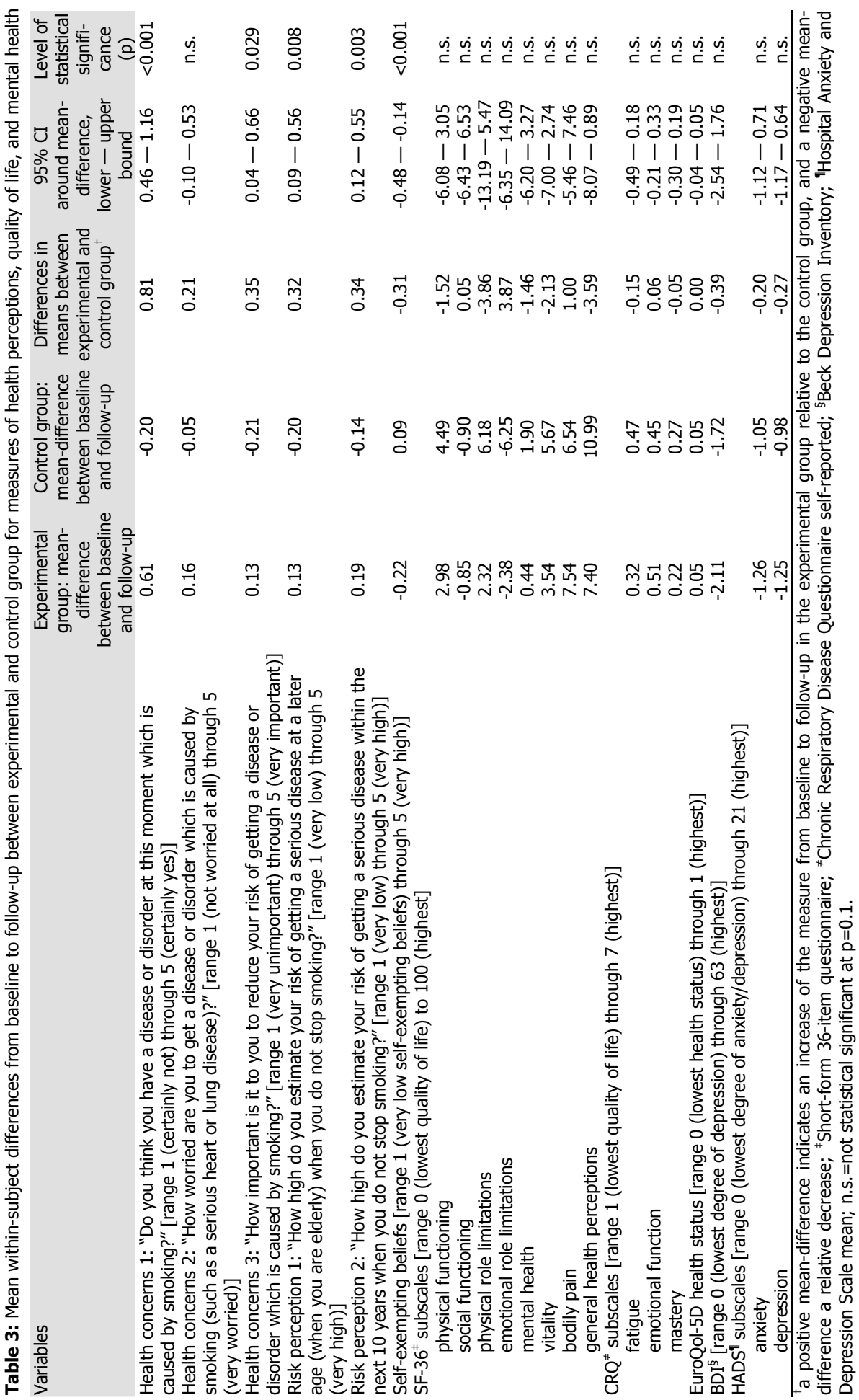


Table 4: Multiple logistic regression modelling the odds of abstinence from smoking for adjusted mediators Independent variables included $(\beta)$

$\operatorname{Exp}(\beta) \quad 95 \%$ confidence interval around $\operatorname{Exp}(\beta)$; lower - upper

Model 1

Intervention (control group=reference)

1.67

$0.97-2.87$

$0.43-1.73$

$0.94-2.19$

$0.25-0.85$

$1.12-1.63$

1.43

0.46

1.35

Self-exficacy ${ }^{\#}$

Model 2 adjusted for baseline characteristics ${ }^{\dagger}$

Intervention (control group = reference)

Risk perceptions $2^{\S}$

Self-exempting beliefs ${ }^{\sharp}$

Self-efficacy ${ }^{\#}$

0.78

1.85

0.41

1.40

§"How high do you estimate your risk of getting a serious disease within the next 10 years when you do not stop smoking?"; ${ }^{\ngtr}$ range from 1 (very low self-exempting beliefs) through 5 (very high); "How high do you consider your chance to succeed in stopping smoking?" [range from 0 (very low chance) through 10 (very high)]; ${ }^{\dagger}$ adjusted for age, sex, educational background, nicotine dependence (FTND), motivation to quit, number of previous quit attempts, airflow limitation (FEV 1 post-bd. \%pred.), and the subscale "physical role limitations" of the SF-36. 


\section{APPENDIX}

Questions measuring "health concerns"

Health concerns 1: "Do you think you have a disease or disorder at this moment which is caused by smoking?"

certainly not probably not indecisive/don't know probably yes certainly yes

Health concerns 2: "How worried are you to get a disease or disorder which is caused by smoking (such as a serious heart or lung disease)?

not worried at all not worried indecisive/don't know worried

Health concerns 3: "How important is it to you to reduce your risk of getting a disease or disorder which is caused by smoking?

very unimportant

unimportant

indecisive/don't know

important

very important

Scoring:

1

2

3

4

5

Questions measuring "risk perceptions"

Risk perception 1: "How high do you estimate your risk of getting a serious disease at a later age (when you are elderly) when you do not stop smoking?

very low low indecisive/don't know $\quad$ high $\quad$ very high

Risk perception 2: "How high do you estimate your risk of getting a serious disease within the next 10 years when you do not stop smoking?

very low low indecisive/don't know high

Scoring:

口

Scoring:

12

2

4

5

Questions measuring "self-exempting beliefs"

In how far do you agree or disagree with the following statements?

totally disagree $\begin{gathered}\text { indecisive / agree totally agree } \\ \text { don't know }\end{gathered}$
disagree

The scientific evidence that smoking is

harmful is not convincing.

Smoking is possibly not very harmful because

many smokers live long.

I would need to smoke far more to jeopardize my health.

The harmful effects can be cleared away, e.g., by eating healthy or exercising regularly.

My body is by nature not susceptible to the harmful effects of smoking.

Smoking is not more harmful than many other things people do.

Everything causes cancer nowadays.

Smoking light cigarettes is not harmful.

Only heavy smoking (20 cigarettes a day or more) is harmful.

Scoring:

$\begin{array}{lllll}\square & \square & \square & \square & \square \\ \square & \square & \square & \square & \square \\ \square & \square & \square & \square & \square \\ \square & \square & \square & \square & \square \\ \square & \square & \square & \square & \square \\ \square & \square & \square & \square & \square \\ \square & \square & \square & \square & \square \\ \square & \square & \square & \square & \square \\ \square & \square & \square & \square & \square \\ 1 & 2 & 3 & 4 & 5\end{array}$




\section{REFERENCES}

1 Global Initiative for Chronic Obstructive Lung Disease. Global strategy for the diagnosis, management, and prevention of chronic pulmonary disease. Executive summary (http://www.goldcopd.com): NHLBI / WHO, 2006

2 World Health Organisation (WHO). The World Health Report. Reducing risks, promoting healthy life. Geneva: WHO, 2002

3 Murray CJ, Lopez AD. Alternative projections of mortality and disability by cause 1990-2020: Global Burden of Disease Study. Lancet 1997; 349:1498-1504

4 Pauwels RA, Buist AS, Calverley PM, et al. Global strategy for the diagnosis, management, and prevention of chronic obstructive pulmonary disease. NHLBI/WHO Global Initiative for Chronic Obstructive Lung Disease (GOLD) Workshop summary. Am J Respir Crit Care Med 2001; 163:1256-1276

5 Anthonisen NR, Connett JE, Kiley JP, et al. Effects of smoking intervention and the use of an inhaled anticholinergic bronchodilator on the rate of decline of FEV1. The Lung Health Study. J Am Med Assoc 1994; 272:1497-1505

6 McBride CM, Emmons KM, Lipkus IM. Understanding the potential of teachable moments: the case of smoking cessation. Health Educ Res 2003; 18:156-170

7 Wilt TJ, Niewoehner D, Kane RL, et al. Spirometry as a motivational tool to improve smoking cessation rates: a systematic review of the literature. Nicotine Tob Res 2007; 9:21-32

8 Wilt TJ, Niewoehner D, Kim C-B, et al. Use of Spirometry for case finding, diagnosis, and management of chronic obstructive pulmonary disease (COPD). Evidence Report/Technology Assessment No. 121 (Prepared by the Minnesota Evidence-based Practice Center under Contract No. 290-02-0009.) AHRQ Publication No. 05-E017-2. Rockville, MD.: Agency for Healthcare Research and Quality, 2005

9 Boushey $\mathrm{H}$, Enright $\mathrm{P}$, Samet J. Spirometry for Chronic Obstructive Pulmonary Disease Case Finding in Primary Care? Am J Respir Crit Care Med 2005; 172:1481-1482

10 Aveyard P, Brown K, Saunders C, et al. Weekly versus basic smoking cessation support in primary care: a randomised controlled trial. Thorax 2007; 62:898-903

11 Parkes G, Greenhalgh T, Griffin M, et al. Effect on smoking quit rate of telling patients their lung age: the Step2quit randomised controlled trial. BMJ 2008; 336:598-600

12 Kotz D, Huibers MJ, Vos R, et al. Principles of confrontational counselling in smokers with chronic obstructive pulmonary disease (COPD). Med Hypotheses 2008; 70:384-386

13 Kotz D, Wesseling GJ, Huibers $\mathrm{MJH}$, et al. Efficacy of confrontational counselling for smoking cessation in smokers with previously undiagnosed mild to moderate airflow limitation: study protocol of a randomized controlled trial. BMC Public Health 2007; 7:332

14 Miller MR, Hankinson J, Brusasco V, et al. Standardisation of spirometry. Eur Respir J 2005; 26:319-338

15 Miller MR, Crapo R, Hankinson J, et al. General considerations for lung function testing. Eur Respir J 2005; 26:153-161

16 STIVORO. De L-MIS: stoppen met roken voor longpatiënten. Handleiding voor longverpleegkundigen. [The L-MIS: smoking cessation in lung patients. Strategy for respiratory nurses]. Den Haag: STIVORO, 2003 17 Hughes JR, Stead LF, Lancaster T. Antidepressants for smoking cessation. Cochrane Database Syst Rev 2004

18 Wagena EJ, Knipschild P, Zeegers MPA. Should nortriptyline be used as a first-line aid to help smokers quit? Results from a systematic review and meta-analysis. Addiction 2005; 100:317-326

19 Ware JE, Jr., Sherbourne CD. The MOS 36-item short-form health survey (SF-36). I. Conceptual framework and item selection. Med Care 1992; 30:473-483

20 McHorney CA, Ware JE, Raczek AE. The MOS 36-item short-form health survey (SF-36): II. Psychometric and clinical tests of validity in measuring physical and mental health constructs. Med Care 1993; 31:247-263

21 Williams JEA, Singh SJ, Sewell L, et al. Development of a self-reported Chronic Respiratory Questionnaire (CRQ-SR). Thorax 2001; 56:954-959

22 Guyatt GH, Berman LB, Townsend M, et al. A measure of quality of life for clinical trials in chronic lung disease. Thorax 1987; 42:773-778

23 The EuroQol Group. EuroQol--a new facility for the measurement of health-related quality of life. Health policy Amsterdam, Netherlands 1990; 16:199-208

24 Brooks R, the EuroQol Group. EuroQol: the current state of play. Health Policy 1996; 37:53-72 
25 Beck AT, Ward $\mathrm{CH}$, Mendelson $\mathrm{M}$, et al. An inventory for measuring depression. Arch Gen Psychiatry $1961 ; 4: 561-571$

26 Zigmond AS, Snaith RP. The Hospital Anxiety and Depression Scale. Acta Psyciatr Scan 1983; 67:361-370

27 Spinhoven PH, Ormel J, Sloekers PPA, et al. A validation of the Hospital Anxiety and Depression Scale (HADS) in different groups of Dutch subjects. Psychological Medicine 1997; 27:363-370

28 Bjelland I, Dahl AA, Tangen Haug T, et al. The validity of the Hospital Anxiety and Depression Scale. An updated literature review. J Psychosom Res 2002; 52:69-77

29 Langone JJ, Gjika HB, Van Vunakis H. Nicotine and its metabolites. Radioimmunoassays for nicotine and cotinine. Biochemistry 1973; 12:5025-5030

30 SRNT Subcommittee on Biochemical Verification. Biochemical verification of tobacco use and cessation. Nicotine Tob Res 2002; 4:149-159

31 Baron RM, Kenny DA. The moderator-mediator variable distinction in social psychological research: conceptual, strategic, and statistical considerations. J Pers Soc Psychol 1986; 51:1173-1182

32 Buffels J, Degryse J, Decramer M, et al. Spirometry and smoking cessation advice in general practice: a randomised clinical trial. Respir Med 2006; 100:2012-2017

33 West R, Hajek P, Stead L, et al. Outcome criteria in smoking cessation trials: proposal for a common standard. Addiction 2005; 100:299-303

34 Ruiter RAC, Abraham C, Kok G. Scary warnings and rational precautions: A review of the psychology of fear appeals. Psychol Health 2001; 16:613-630

35 Witte K, Allen M. A meta-analysis of fear appeals: Implications for effective public health campaigns. Health Educ Behav 2000; 27:591-615

36 Milne S, Sheeran P, Orbell S. Prediction and intervention in health-related behavior: A meta-analytic review of protection motivation theory. J Appl Soc Psychol 2000; 30:106-143

37 Peretti-Watel P, Constance J, Guilbert P, et al. Smoking too few cigarettes to be at risk? Smokers' perceptions of risk and risk denial, a French survey. Tob Control 2007; 16:351-356

38 Halpern MT. Effect of smoking characteristics on cognitive dissonance in current and former smokers. Addict Behav 1994; 19:209-217

39 McMaster C, Lee C. Cognitive dissonance in tobacco smokers. Addict Behav 1991; 16:349-353

40 Chapman S, Wong WL, Smith W. Self-exempting beliefs about smoking and health: differences between smokers and ex-smokers. Am J Public Health 1993; 83

41 Lee C. Perceptions of immunity to disease in adult smokers. J Behav Med 1997; 12:267-277

42 McCoy SB, Gibbons FX, Reis TJ, et al. Perceptions of smoking risk as a function of smoking status. J Behav Med 1992; 15:469-488

43 Festinger L. A theory of cognitive dissonance. Stanford: Stanford University Press, 1957

44 Morgan JF. p Value fetishism and use of the Bonferroni adjustment. Evid Based Ment Health 2007; 10:34-35

45 Wainer H, Robinson DH. Research News and Comments - Shaping Up the Practice of Null Hypothesis Significance Testing. Educational Researcher 2003; 32:22-30

46 Altman DG, Bland JM. Absence of evidence is not evidence of absence. Brit Med J 1995; 311:485

47 Nakagawa S, Cuthill IC. Effect size, confidence interval and statistical significance: a practical guide for biologists. Biol Rev Camb Philos Soc 2007; 82:591-605

48 Balluerka N, Gómez J, Hidalgo D. The controversy over Null Hypothesis Significance Testing revisited. Methodology 2005; 1:55-70

49 Bize R, Burnand B, Mueller Y, et al. Biomedical risk assessment as an aid for smoking cessation. Cochrane Database Syst Rev 2005

50 Bednarek M, Gorecka D, Wielgomas J, et al. Smokers with airway obstruction are more likely to quit smoking. Thorax 2006; 61:869-873 



\section{Chapter 9}

\section{Ethical analysis of the justifiability of labelling with COPD for smoking cessation.}

Daniel Kotz
Rein Vos $^{2}$

Marcus J.H. Huibers ${ }^{3}$

Submitted for publication

${ }^{1}$ Department of General Practice, School for Public Health and Primary Care (CAPHRI), Maastricht University Medical Centre, Maastricht, the Netherlands. ${ }^{2}$ Department of Health Ethics and Philosophy, School for Public Health and Primary Care (CAPHRI). ${ }^{3}$ Department of Clinical Psychological Science, Maastricht University, Maastricht, the Netherlands 


\section{ABSTRACT}

\section{Background}

Spirometry for early detection of chronic obstructive pulmonary disease (COPD) and smoking cessation is criticized because of the potential negative effects of labelling with disease.

\section{Aim}

To assess the opinions of smokers on the effectiveness of spirometry for smoking cessation, the justification of early detection of airflow limitation in smokers, and the impact of confrontation with COPD.

\section{Design}

Qualitative study with data from a randomized controlled trial.

\section{Setting}

General population of Dutch and Belgian Limburg, the Netherlands.

\section{Methods}

We conducted semi-structured ethical exit interviews in 205 smokers who were motivated to quit smoking, had no prior diagnosis of COPD, but were detected with airflow limitation by means of spirometry. They received either (1) counselling including labelling with COPD in combination with nortriptyline for smoking cessation, (2) counselling excluding labelling with COPD in combination with nortriptyline for smoking cessation, or (3) care as usual for smoking cessation by the general practitioner excluding labelling with COPD.

\section{Results}

One hundred seventy-seven participants (86\%) agreed or completely agreed that it is justified to measure lung function in heavy smokers. These participants argued that measuring lung function raises consciousness about the negative effects of smoking, helps to prevent disease, or increases motivation to stop smoking. Most of the 18 participants who disagreed argued that routinely measuring lung function in smokers would interfere with one's freedom of choice.

\section{Conclusions}

Labelling with disease is probably a less important issue in the discussion about the pros and cons of early detection of COPD. 


\section{How this fits in}

The use of spirometry for early detection of chronic obstructive pulmonary disease (COPD) and smoking cessation is criticized because of the potential negative effects of labelling with disease. Whereas many authors have raised ethical concerns, empirical data of patients' perspectives in this debate are rare. The results from our trial on early detection and labelling with COPD for smoking cessation show that the vast majority of smokers finds it justified to measure lung function in heavy smokers for the sake of consciousness-raising about the negative effects of smoking, prevention of disease, or increase of motivation to stop smoking. Labelling with disease is probably a less important issue in the discussion about the pros and cons of early detection of COPD.

\section{INTRODUCTION}

Chronic obstructive pulmonary disease (COPD) is a preventable and treatable disease which is characterized by airflow limitation that is not fully reversible ${ }^{1}$. It is currently the fifth leading cause of death worldwide ${ }^{2}$, and a further increase in mortality is predicted in the coming decades. ${ }^{3}$ Smoking is by far the most important risk factor for COPD, and smoking cessation is the single most effective way to reduce the risk of developing COPD and to affect the outcome in patients at all stages of the disease. ${ }^{4,5}$ Because of the irreversible and progressive nature of the disease, early intervention in COPD is important. However, COPD is usually underestimated by patients and by health-care providers, because most patients do not present with respiratory symptoms, and those who do are not always diagnosed as such. ${ }^{6}$ An issue of debate is the use of spirometry for early detection of COPD. ${ }^{7-10}$ Its ethical aspects are the subjects of this paper.

"Confrontational counselling"11 is a patient-centred approach which involves confronting smokers with the consequences of their addiction, i.e. labelling smokers with COPD. Labelling with disease - confronting healthy subjects with a previously undetected or unrecognized disease status - has for long been an issue of debate in the medical field. In most cases, the debate has focused on the potential adverse effects of labelling, ranging from the ethical aspects of screening for an incurable disease, thereby advancing the onset of life years afflicted by disease, to the risks of stigmatization and exclusion involved in genetic screening programmes ${ }^{12}$, and to the impact the diagnosis of a contested illness such as chronic fatigue syndrome might have ${ }^{13}$. With regard to early detection of COPD by means of spirometry, it has been argued that widespread spirometry is likely to label a large number of individuals with disease, and that this results in considerable testing, treatment costs, and healthcare resource utilization. ${ }^{14-16}$ However, it is also possible that the act of labelling can be used as a motivational tool to change unhealthy behaviour (e.g., smoking). 
Many authors have raised ethical concerns with regard to early detection of COPD and our claim is not that our confrontational counselling approach is ethically the correct stance. Although we think it is a pragmatic, feasible, and legitimate method, our major aim in this paper is to let the smokers receiving smoking cessation treatment speak for themselves. This is relevant by itself, since empirical data of patients' perspectives in this debate are rare. As part of a randomized controlled trial on early detection of airflow limitation and subsequent smoking cessation treatment ${ }^{17}$, we conducted semi-structured ethical exit interviews on this subject with the study participants. The objective of this paper is to assess the opinions of these participants on the effectiveness of spirometry for smoking cessation, the justification of early detection of airflow limitation in smokers, and the impact of confrontation with COPD.

\section{METHODS}

We used data from a randomized controlled trial confrontational counselling with previously undiagnosed COPD for smoking cessation. A detailed description of the trial protocol has been published elsewhere. ${ }^{17}$ In brief, current smokers aged 35 through 70 years who were motivated to quit smoking were invited to take part in a study on individual counselling and medication for smoking cessation. Participants were excluded when they reported to have COPD, chronic bronchitis, asthma, or asthmatic bronchitis. The participant information sheet did not include any information about early detection and confrontation with COPD and the differences in counselling between the experimental and the control group. Subjects were eligible if they had mild or moderate airflow limitation defined as post-bronchodilator (postbd.) $\mathrm{FEV}_{1} / \mathrm{FVC}<70 \%$ in combination with post-bd. $\mathrm{FEV}_{1} \geq 50 \%$ of predicted value. ${ }^{1}$ The results of spirometry were not discussed at that moment. Subjects with severe or very severe COPD were not included but referred to their GP.

Participants from both the experimental group and control group 1 received equally intensive counselling delivered by a respiratory nurse combined with nortriptyline for smoking cessation, based on a national protocol. ${ }^{18}$ Specific elements of "confrontational counselling"11 were added to the protocol in the experimental group which discriminated the treatment from that in control group 1. Most importantly, only these subjects were confronted with the results from spirometry and labelled with the disease COPD. Participants from control group 2 were referred to their own GP for primary "care as usual" for smoking cessation, which involves the use of a protocol for low intensity health education and promotion, the so-called "H-MIS". ${ }^{19}$ 
All participants were followed-up for 12 months after their target quit date. During the 12-month follow-up visit, a research assistant conducted a semi-structured ethical exit interview on the issue of early detection of COPD and confrontation with spirometry. The interview consisted of four statements (box 1) the assistant read out to the participant. The participant was asked to rate the statement on a 5-point Likert scale ranging from "completely disagree" (score 1) through "completely agree" (score 5). Subsequently, the assistant stimulated the participant to elaborate on his/her opinion and to formulate arguments. The assistant wrote down all arguments in the participant's own words, providing transcripts of "short hand descriptions" for the data analysis.

\section{Data analysis}

The quantitative data from the Likert scales were used to calculate means and standard deviations. For the statistical testing, participants scoring 1 ("completely disagree") and 2 ("disagree") were combined into one group and participants scoring 4 ("agree") and 5 ("completely agree") into another group (participants scoring 3 "neutral" were omitted). We found it justifiable to omit "neutral" responses because they were rare $(1-6 \%)$ and because we wanted to compare the two opposite sides of viewpoint. Chi-square tests were used to test statistical differences in proportions of subjects who agreed versus disagreed. All these analyses were performed in SPSS.

The analysis of the qualitative data was performed according to the method of grounded theory as developed by Glaser and Strauss ${ }^{20}$ and further extended by Strauss and Corbin ${ }^{21,22}$. Grounded theory uses the constant comparative method of data analysis. In this approach coding is used to conceptualize data into patterns or concepts. ${ }^{23}$ Participants' arguments were extracted from the transcripts, analyses and finally aggregated into six clusters: freedom of choice, consciousness-raising, regular health check-up, prevention of disease, motivation to quit smoking, and other. All analyses were performed by two researchers (DK and RV). Both investigators brought different skills and expertise to the analysis, benefiting the analysis and interpretation form divergent perspectives. ${ }^{23}$

\section{RESULTS}

A total of 296 participants were included into the trial in the period from February 2005 through January 2007. Two hundred and five participants (69\%) completed the 12-month follow-up measurement; 85 out of 116 participants (73\%) randomized to the experimental group, 80 out of 112 participants (71\%) randomized to control group 1, and 40 out of 68 participants (59\%) randomized to control group 2 . The statistical test for difference in lost to follow-up across the three groups was not significant (Chi-square $=4.60, \mathrm{df}=2, \mathrm{p}=0.100$ ). The baseline characteristics of the 
participants who entered the analyses are shown in table 1 . These did not differ from the characteristics of the full sample of 296 participants who were randomized. Responses to the three questions about health concerns showed that, on average, participants were unsure about having a smoking-related disease (health concern 1), but that they were worried about getting such a disease (health concern 2) and that they found it very important to reduce their risk of getting such a disease (health concern 3).

\section{Statement 1: Does measuring lung function have a favourable effect on the attempt to quit smoking?}

Ninety-four participants (46\%) agreed or completely agreed that measuring lung function positively influenced their quit attempt (figure 1). Ninety-seven participants $(47 \%)$ disagreed or completely disagreed and $13(6 \%)$ were neutral. More participants from the experimental group (61\%) than from control group 1 (33\%) and control group 2 (51\%) agreed (table 2$)$.

Within the 94 participants who agreed with the statement, $16 \%(\mathrm{~N}=15)$ argued that confrontation with objective evidence of respiratory illness influenced their quit attempt, $12 \%(\mathrm{~N}=11)$ that the measurement confirmed a presumption that something was wrong with their lungs, and $18 \%(\mathrm{~N}=17)$ that they had difficulties with performing the lung function test. Another frequently mentioned argument was taking part in a serious research project as a reason to quit $(\mathrm{N}=17,18 \%)$.

Within the 97 participants who disagreed with statement 1, 57\% ( $\mathrm{N}=55)$ argued that spirometry itself without discussing the results did not have an effect. The proportion of participants using this argument was higher in control group $1(34 \%)$ than in the experimental group (20\%) and control group 2 (28\%). In control group 1, the respiratory nurses were instructed not to discuss the results from spirometry during the counselling session.

\section{Statement 2: Is it justifiable to measure lung function in heavy smokers?}

One hundred seventy-seven participants (86\%) agreed or completely agreed that it is justified to measure lung function in smokers (figure 2). Eighteen participants (9\%) disagreed or completely disagreed and 9 (4\%) were neutral. Participants who agreed were more likely to be smokers, i.e. participants who were not successful in quitting smoking during the 12 month follow-up (table 2). A selection of quotes relating to frequently mentioned arguments is presented in table 3.

Within the 177 participants who agreed with the statement, 57\% ( $\mathrm{N}=100)$ argued that measuring lung function raises consciousness about the negative effects of smoking, and 34\% ( $\mathrm{N}=60)$ justified it with early detection and prevention of disease. Using lung function as part of a regular health check-up was an argument brought 
up by $14 \%(\mathrm{~N}=24)$, and $21 \%(\mathrm{~N}=37)$ found that it was justified because it increases the motivation to stop smoking. ${ }^{\ddagger}$ The mean number of arguments used was about the same (mean range 1.6-1.7) in all three intervention groups. There was also no difference between the groups in the number of arguments from the different clusters.

Within the 18 participants who disagreed with statement 2, 89\% ( $\mathrm{N}=16)$ argued that routinely measuring lung function in smokers would interfere with one's freedom of choice.

\section{Statements 3 and 4: What impact does confrontation with COPD have?}

Statements 3 and 4 refer only to participants from the experimental group. Solely in this group, participants were confronted with the results from spirometry and the fact that they have airflow limitation. Of the 85 participants who entered the analysis, $83(98 \%)$ agreed that it was justified that the respiratory nurse confronted them with COPD, whereas only one participant disagreed (1\%). Also, 78 participants (92\%) agreed that the confrontation with spirometry helped them to stop smoking. Five participants (6\%) disagreed and one (1\%) was neutral. These answers were not associated with self-reported smoking status at follow-up, but more participants with a worse lung function (lower half: $\mathrm{FEV}_{1}<84.1 \%$ ) stated that confrontation with spirometry motivated them to stop smoking (table 2).

\section{DISCUSSION}

Within the framework of a randomized controlled trial on smoking cessation treatment in smokers with previously undiagnosed COPD, and with data from semistructured exit interviews in 205 participants, we performed an analysis of ethical issues regarding early detection of aifflow limitation and confrontation with COPD. We provided new empirical data of patients' perspectives about these issues. The vast majority of participants found it justified to measure lung function in smokers and to confront smokers with the results from spirometry. This opinion was supported by arguments about consciousness-raising, prevention of disease, regular health check-up, and motivation to stop smoking.

Many authors have raised ethical concerns with regard to early detection of COPD, basically because widespread spirometry would be likely to label a large number of individuals with disease. The major aim of our analyses was to let smokers who experienced early detection and confrontation with COPD speak for themselves. The very few smokers who did not find it justified to measure lung function in smokers

₹ Note that the proportions do not sum-up $100 \%$ because participants could use multiple arguments (a maximum 5). 
saw this approach as an interference with one's freedom of choice. The majority of smokers, however, were in favour of measuring lung function in smokers in general, basically because it helps to prevent disease and allows early intervention, and because it might motivate smoking cessation due to consciousness-raising about the negative effects of smoking. This suggests, that labelling with disease is not found as problematic by the people to which it applies (the smokers with mild to moderate COPD) as by some authors. ${ }^{14-16,24}$ The number of arguments used between the three intervention groups were indifferent. The fact that the experimental group (the only group that was confronted with declined lung function as part of the smoking cessation counselling) did not use more arguments than the two other groups indicate that there was no learning effect or, formulated more sharply, no effect of medicalization.

Less than half of all participants agreed that measuring lung function positively influenced their quit attempt, regardless of smoking status. However, within participants from the experimental group, nearly everybody agreed that confrontation with lung function was helpful for their quit attempt. Interestingly, relatively more smokers with worse lung function agreed, which may indicate a greater susceptibility to confrontational counselling in smokers with more advanced COPD. These viewpoints and the arguments used for underpinning suggest that merely measuring lung function does not promote smoking cessation, but that confrontation with lung function should be integrated into a smoking cessation counselling programme. This confirms our hypothesis that altering irrational cognitions smokers use to legitimatize their behaviour is not likely to be achieved by brief advice. ${ }^{11}$

The smokers participating in this trial were all characterized by a high motivation to quit smoking, by the presence of concerns about getting a smoking related disease in the future and by the urge of reducing that risk. This is important when interpreting the results of this study, because smokers who are not motivated to quit smoking or who are not concerned about their health are likely to have different attitudes towards early detection and labelling with disease. We do think, however, that our study population represents an important group of people, as most smokers want to quit smoking and many of them because of health concerns. We created a clinical context for participants from the experimental group and control group 1 in which they were treated individually and with an intensive interaction with a respiratory nurse, which does not reflect current care as usual for smoking cessation. Thus, this paper does not give an answer as to whether smokers find a more population-based, screening approach of COPD ethically acceptable. This paper does also not represent the views of ex- or non-smokers. However, early detection of COPD in this group is probably not ethical because smoking cessation is the only effective therapy for progression of the disease, leaving not much to offer to those who already quit smoking (despite from symptom relief therapy with medication). 
We are aware of the limitations of our study, particularly using short exit interviews and short hand descriptions of the answers smokers provided in these sessions delivered by respiratory nurses - constraining more in-depth qualitative research, which is impossible due to the demands of performing such a clinical trial. Part of the participants who were randomized at the beginning of the trial were lost to follow-up after 12 months. Though the characteristics of the sample that entered this analysis were comparable with the full sample of randomized participants it may be that participants who were lost to follow-up had different attitudes. We suspect that smokers who relapsed to smoking were less likely to complete the follow-up visits, but smoking status was not an important factor associated with the responses. Despite these limitations, we hope to provide interesting data on the ethical issues involved and to the ethical debate on confrontational forms of counselling as in our case the early detection of COPD and the confrontation with such a diagnosis in order to change life style practices.

We conclude that smokers find measuring lung function in smokers and labelling with COPD justifiable for the sake of early detection and prevention of disease. It may also facilitate smoking cessation, but only in the context of an individual counselling approach. Labelling with disease is probably a less important issue in the discussion about the pros and cons of early detection of COPD. 
Box 1: Statements on the issue of early detection of COPD and confrontation with spirometry

Statement 1: "The fact that my lung function was measured at the start of this study has had a favourable effect on my attempt to quit smoking."

Statement 2: "It is justifiable to measure the lung function of people who have been heavy smokers for a long time."

Statement 3: "The respiratory nurse was right to confront me with this lung disease (COPD) during the study."

Statement 4: "The fact that the pulmonary nurse discussed the lung function measurement with me in order to confront me with COPD has helped me in my attempt to quit smoking."

Table 1: Baseline characteristics of the respondents to the ethical exit-interview

\begin{tabular}{|c|c|}
\hline Characteristics & $\mathrm{N}=205$ \\
\hline \multicolumn{2}{|l|}{ Intervention group, $\mathrm{N}(\%)$} \\
\hline Experimental group & $85(39.0)$ \\
\hline Control group 1 & $80(39.0)$ \\
\hline Control group 2 & $40(19.5)$ \\
\hline Years of age & $54.5(7.2)$ \\
\hline Male sex, N (\%) & $85(61.2)$ \\
\hline Educational level $^{\dagger}$ & $4.0(1.9)$ \\
\hline $\mathrm{FTND}^{\ddagger}$, mean (SD) & $4.5(1.5)$ \\
\hline $\mathrm{FEV}_{1}$ post-bd.\% pred ${ }^{q}$ & $82.3(15.8)$ \\
\hline $\begin{array}{l}\text { Health concerns } 1 \text { : "Do you think you have a disease or disorder at this moment } \\
\text { which is caused by smoking?" [range from } 1 \text { (certainly not) through } 5 \text { (certainly } \\
\text { yes)] }\end{array}$ & $2.8(1.1)$ \\
\hline $\begin{array}{l}\text { Health concerns 2: "How worried are you to get a disease or disorder which is } \\
\text { caused by smoking (such as a serious heart or lung disease)?" [range from } 1 \text { (not } \\
\text { worried at all) through } 5 \text { (very worried)] }\end{array}$ & $3.9(0.9)$ \\
\hline $\begin{array}{l}\text { Health concerns } 3 \text { : "How important is it to you to reduce your risk of getting a } \\
\text { disease or disorder which is caused by smoking? [range from } 1 \text { (very unimportant) } \\
\text { through } 5 \text { (very important)] }\end{array}$ & $4.4(0.9)$ \\
\hline \multicolumn{2}{|c|}{$\begin{array}{l}\text { Data are presented as mean (SD), unless otherwise indicated. }{ }^{\dagger} \text { Range from } 1 \text { (lowest educational level) } \\
\text { through } 7 \text { (highest). }{ }^{7} \text { Fagerström Test for Nicotine Dependence }{ }^{25} \text { : range from } 0 \text { (lowest level of nicotine } \\
\text { dependence) through } 10 \text { (highest). " Baseline post-bronchodilator forced expiratory volume in one second } \\
\text { (percentage of predicted value). }\end{array}$} \\
\hline
\end{tabular}




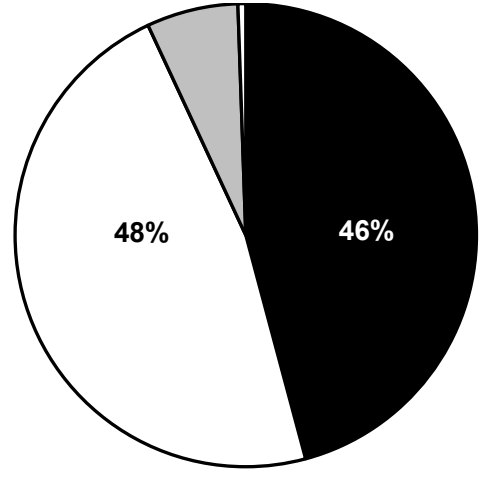

agree $\square$ disagree $\square$ neutral $\square$ missing

Figure 1: Responses to statement 1 "The fact that my lung function was measured at the start of this study has had a favourable effect on my attempt to quit smoking."

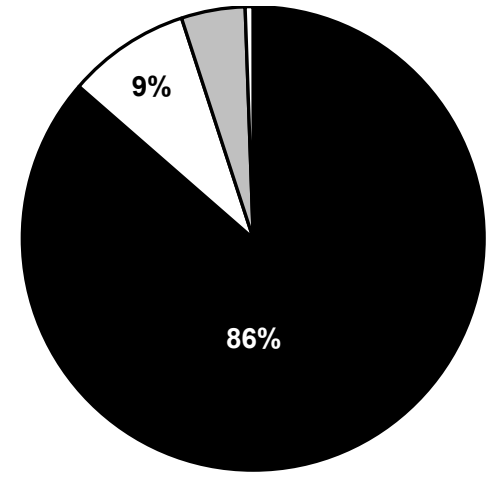

agree $\square$ disagree $\square$ neutral $\square$ missing

Figure 2: Responses to statement 2"It is justifiable to measure the lung function of people who have been heavy smokers for a long time.

Table 2: Numbers (\% within stratum) of participants agreeing ${ }^{\dagger}$ with the four statements, stratified by possible covariates

\begin{tabular}{|c|c|c|c|c|}
\hline Strata & $\begin{array}{c}\text { Statement } 1 \\
\text { "Measuring lung } \\
\text { function had a } \\
\text { favourable effect } \\
\text { on my quit } \\
\text { attempt" }\end{array}$ & $\begin{array}{l}\text { Statement } 2 \\
\text { "It is justifiable to } \\
\text { measure lung } \\
\text { function in heavy } \\
\text { smokers" }\end{array}$ & $\begin{array}{c}\text { Statement } 3^{\ddagger} \\
\text { "The respiratory } \\
\text { nurse was right to } \\
\text { confront me with } \\
\text { COPD" }\end{array}$ & $\begin{array}{l}\text { Statement } 4^{\ddagger} \\
\text { "Confrontation with } \\
\text { my lung function } \\
\text { helped me with my } \\
\text { quit attempt" }\end{array}$ \\
\hline Intervention group & ** & n.s. & & \\
\hline Experimental group & $52(61.2)$ & $72(87.8)$ & 83 (97.6) & 78 (92.9) \\
\hline Control group 1 & $24(33.3)$ & 74 (94.9) & n.a. & n.a. \\
\hline Control group 2 & $18(51.4)$ & $31(86.1)$ & n.a. & n.a. \\
\hline $\begin{array}{l}\text { males } \\
\text { females }\end{array}$ & $\begin{array}{c}\text { n.s. } \\
61(48.0)\end{array}$ & $\begin{array}{c}\text { n.s. } \\
120(93.8)\end{array}$ & $\begin{array}{c}\text { n.s. } \\
53(98.1)\end{array}$ & $\begin{array}{c}\text { n.s. } \\
48(90.6)\end{array}$ \\
\hline $\begin{array}{l}\text { females } \\
\text { Self-reported smoking status at }\end{array}$ & $33(50.8)$ & $57(83.8)$ & $30(96.8)$ & $30(96.8)$ \\
\hline $\begin{array}{l}\text { Self-reported smoking status at } \\
12 \text { month follow-up } \\
\text { smoker } \\
\text { non-smoker }\end{array}$ & $\begin{array}{c}\text { n.s. } \\
48(46.6) \\
46(51.7)\end{array}$ & $\begin{array}{c}* \\
102(95.3) \\
75(84.3)\end{array}$ & $\begin{array}{c}\text { n.s. } \\
41(100) \\
42(95.5)\end{array}$ & $\begin{array}{c}\text { n.s. } \\
38(95.0) \\
40(90.9)\end{array}$ \\
\hline $\begin{array}{l}\text { Educational level } \\
\text { lower } 50 \% \text { (level 1-3) } \\
\text { higher } 50 \% \text { (level } 4-7 \text { ) }\end{array}$ & $\begin{array}{l}\text { n.s. } \\
43(48.9) \\
51(49.0)\end{array}$ & $\begin{array}{l}\text { n.s. } \\
80(87.0) \\
97(93.3)\end{array}$ & $\begin{array}{l}\text { n.s. } \\
38(95.0) \\
45(100)\end{array}$ & $\begin{array}{l}\text { n.s. } \\
37(94.9) \\
41(91.1)\end{array}$ \\
\hline $\begin{array}{l}\mathrm{FEV}_{1} \text { post-bd. } \% \text { pred at } \\
\text { baseline } \\
\text { lower } 50 \%\left(\mathrm{FEV}_{1}<84.1\right) \\
\text { higher } 50 \%\left(\mathrm{FEV}_{1}>=84.1\right)\end{array}$ & $\begin{array}{c}\text { n.s. } \\
54(50.9) \\
40(46.5)\end{array}$ & $\begin{array}{c}\text { n.s. } \\
75(86.2) \\
102(93.6)\end{array}$ & $\begin{array}{c}\text { n.s. } \\
44(95.7) \\
39(100)\end{array}$ & $\begin{array}{c}* \\
44(97.8) \\
34(87.2)\end{array}$ \\
\hline
\end{tabular}

${ }^{\dagger}$ The original answering scale ranged from 1 (completely disagree) through 5 (completely agree). Participants who agreed or completely agreed (scores 4 and 5) were clustered. The Chi-square tests were used to test differences in proportion of subjects who agreed with the proportion of subjects who disagreed (scores 1 and 2). Subjects who gave a neutral answer (score 3$)$ were excluded from the statistical testing $(\mathrm{N}=13(6.3 \%)$, $\mathrm{N}=9(4.4 \%), \mathrm{N}=0, \mathrm{~N}=1(1.1 \%)$ for the statements $1,2,3$, and 4 respectively). ${ }^{\ddagger}$ Questions 3 and 4 refer to the experimental group only. $\mathrm{RN}=$ respiratory nurse. ${ }^{*} \mathrm{p}<0.05, * * \mathrm{p}<0.01$, n.s. $=$ no statistical significant difference $(p \geq 0.05)$, n.a.=not applicable. 
Table 3: Selection of quotes from participants arguing for or against statement 2 "It is justifiable to measure the lung function of people who have been heavy smokers for a long time." Agree Disagree

"The fact that you smoke doesn't imply that you have "People should be given a free choice in this respect." to disregard your health status."

"It should even be compulsory. People tell you 'smoking is bad for you', but in fact, far too little fuss is being made about it."

"If you have no complaints, there's no point."

"It would be good to do these tests as a matter of routine, as a preventive measure to detect diseases."

"It's always your own choice. Some people have no problem at all with the fact that they smoke."

"It's a good opportunity to detect diseases at an early stage, which can motivate people to stop smoking."

"People have to be forcefully confronted with the facts. You can't be too clear about this!"

"There's no harm in it anyway."

"It's a good idea to monitor the process, since smoking puts your airways at risk."

"If my lungs should turn out to have deteriorated from smoking, I would definitely change my ways. It's a 'must', for the sake of your own health."

"Everyone knows smoking is bad for you, but the message is probably more convincing if it's backed up by figures."

"It's better to look forward than to look backward. If you wait too long, you might one day think 'if only I'd acted sooner'."

"Many people are unaware of the real consequences of smoking."

"You want to know the state of your health, so that you take action at an early stage if necessary."

"Yes, it's the moment of truth."

"It's your own choice to smoke, so your GP is allowed to measure your pulmonary function."

"Smokers often won't admit that they're addicted, but such tests force you to face facts."

"If you're addicted, you don't know your limits. Such a confrontation makes you aware of them."

"You can't fool yourself. It's a bad habit, and such a clear confrontation may be that little extra push you need to finally quit."
"It's a form of discrimination."

"That's for people to decide for themselves!"

"Whether someone smokes or not is up to them."

"Everybody knows that smoking is bad for your health, especially for the airways. Routine measurements, that's overdoing it."

"Only if you have any complaints, at your GP's advice."

"You're old and wise enough to know it's bad for you."

"I really think it's patronising, doctors are interfering

too much. The media, doctors, etc.: we're being swamped with rules." 


\section{REFERENCES}

1 Global Initiative for Chronic Obstructive Lung Disease. Global strategy for the diagnosis, management, and prevention of chronic pulmonary disease. Executive summary (http://www.goldcopd.com): NHLBI / WHO, 2006

2 World Health Organisation (WHO). The World Health Report. Reducing risks, promoting healthy life. Geneva: WHO, 2002

3 Murray CJ, Lopez AD. Alternative projections of mortality and disability by cause 1990-2020: Global Burden of Disease Study. Lancet 1997; 349:1498-1504

4 Pauwels RA, Buist AS, Calverley PM, et al. Global strategy for the diagnosis, management, and prevention of chronic obstructive pulmonary disease. NHLBI/WHO Global Initiative for Chronic Obstructive Lung Disease (GOLD) Workshop summary. Am J Respir Crit Care Med 2001; 163:1256-1276

5 Anthonisen NR, Connett JE, Kiley JP, et al. Effects of smoking intervention and the use of an inhaled anticholinergic bronchodilator on the rate of decline of FEV1. The Lung Health Study. J Am Med Assoc 1994; 272:1497-1505

6 van Schayck CP, van der Heijden FMMA, van den Boom G, et al. Underdiagnosis of asthma: is the doctor or the patient to blame? The DIMCA project. Thorax 2000; 55:562-565

7 Boushey $\mathrm{H}$, Enright P, Samet J. Spirometry for Chronic Obstructive Pulmonary Disease Case Finding in Primary Care? Am J Respir Crit Care Med 2005; 172:1481-1482

8 Enright PL, Studnicka M, Zielinski J. Spirometry to detect and manage chronic obstructive pulmonary disease and asthma in the primary care setting. Eur Respir Mon 2005; 31:1-14

9 White P. Should we use spirometry in the early detection of COPD? Eur Respir J 2005; 26:558-559

10 Gorecka D, Bednarek M, Nowinski A, et al. Diagnosis of airflow limitation combined with smoking cessation advice increases stop-smoking rate. Chest 2003; 123:1916-1923

11 Kotz D, Huibers MJ, Vos R, et al. Principles of confrontational counselling in smokers with chronic obstructive pulmonary disease (COPD). Med Hypotheses 2008; 70:384-386

12 Markel $\mathrm{H}$. The stigma of disease: implications of genetic screening. Am J Med 1992; 93:209-215

13 Huibers $\mathrm{MJH}$, Wessely S. The act of diagnosis: pros and cons of labelling chronic fatigue syndrome. Psychol Med 2006; 10:1-6

14 Wilt TJ, Niewoehner D, Kim C-B, et al. Use of Spirometry for case finding, diagnosis, and management of chronic obstructive pulmonary disease (COPD). Evidence Report/Technology Assessment No. 121 (Prepared by the Minnesota Evidence-based Practice Center under Contract No. 290-02-0009.) AHRQ Publication No. 05-E017-2. Rockville, MD.: Agency for Healthcare Research and Quality, 2005

15 Enright P. Does screening for COPD by primary care physicians have the potential to cause more harm than good? Chest 2006; 129:833-835

16 Wilt TJ, Niewoehner D, Kane RL, et al. Spirometry as a motivational tool to improve smoking cessation rates: a systematic review of the literature. Nicotine Tob Res 2007; 9:21-32

17 Kotz D, Wesseling GJ, Huibers MJH, et al. Efficacy of confrontational counselling for smoking cessation in smokers with previously undiagnosed mild to moderate airflow limitation: study protocol of a randomized controlled trial. BMC Public Health 2007; 7:332

18 STIVORO. De L-MIS: stoppen met roken voor longpatiënten. Handleiding voor longverpleegkundigen. [The L-MIS: smoking cessation in lung patients. Strategy for respiratory nurses]. Den Haag: STIVORO, 2003

19 Pieterse ME, Seydel ER, DeVries $H$, et al. Effectiveness of a minimal contact smoking cessation program for Dutch general practitioners: a randomized controlled trial. Prev Med 2001; 32:182-190

20 Glaser BG, Strauss AL. The discovery of grounded theory: strategies for qualitative research. Chicago: Aldine, 1967

21 Strauss AL, Corbin JM. Grounded theory: an overview. In: Denzin NK, Lincoln YS, eds. Handbook of qualitative research. Thousand Oaks: Sage, 1994

22 Strauss AL, Corbin JM. Basics of qualitative research. Techniques and procedures for developing grounded theory. 2nd ed. Thousand Oaks: Sage, 1998

23 Polit DF, Beck CT. Nursing research: principles and methods. Philadelphia: Lippincot Williams \& Wilkins, 2004

24 Miller MR. What defines abnormal lung function? Thorax 2007; 62:1107; author reply 1108-1109 
Chapter 9

25 Heatherton TF, Kozlowski LT, Frecker RC, et al. The Fagerström Test for Nicotine Dependence: a revision of the Fagerström Tolerance Questionnaire. Br J Addict 1991; 86:1119-1127 
EPILOGUE 



\section{Chapter 10}

\section{Explaining the social gradient in smoking cessation: it's not in the trying, but in the succeeding}

Daniel Kotz ${ }^{1}$

Robert West ${ }^{2}$

Accepted for publication in Tobacco Control

${ }^{1}$ Department of General Practice, School for Public Health and Primary Care (CAPHRI), Maastricht University Medical Centre, Maastricht, the Netherlands. ${ }^{2}$ Cancer Research UK Health Behaviour Unit, Department of Epidemiology and Public Health, University College London, London, UK 


\section{ABSTRACT}

\section{Objective}

Smokers from more deprived socio-economic groups are less likely to become exsmokers. This study examined how far this is attributable in England to a social gradient in quit attempts, use of aids to cessation, and/or the success of quit attempts.

\section{Design}

Cross-sectional household survey.

\section{Setting}

England.

\section{Participants}

6,950 respondents aged $16+$ who had smoked in the past year of whom 2,983 had tried to stop in the past year and 469 reported having stopped at the time of the survey.

\section{Main outcome measures}

Reported attempts to stop smoking in the past year; the use of stop-smoking medications (nicotine replacement therapy, bupropion or varenicline); use of behavioural support offered by the National Health Service (NHS) Stop Smoking Services; current smoking status.

\section{Results}

There was a large difference in success rates of quit attempts: $20.4 \%$ in social grade $A B$ versus $11.4 \%$ in social grade $E$ of those who made attempts were still not smoking by the time of the survey $(p<0.001)$. However, there was no difference across social grades in the rate of attempts to stop ( $42.7 \%$ in social grade $A B$ to $41.3 \%$ in $E$ ), use of medications to aid cessation ( $46.7 \%$ of those making quit attempts in $A B$ to $50.9 \%$ in E) or use of NHS Stop Smoking Services $(7.0 \%$ of those making quit attempts in $A B$ to $4.8 \%$ in $E$ ).

\section{Conclusions}

Smokers in more deprived socio-economic groups are just as likely as those in higher groups to try to stop and use aids to cessation but there is a strong gradient across socio-economic groups in success, with those in the lowest group being half as likely to succeed compared with the highest. Determining the cause of, and counteracting, this gradient is paramount in reducing health inequalities. 


\section{What is already known about this topic}

There is a strong socio-economic gradient in the rate at which smokers become exsmokers but it is not clear how far this relates to differences in the rate at which they make quit attempts and/or use effective aids to cessation.

\section{What this study adds}

The difference in cessation rates is not attributable to differences in rate of attempts to stop but to differences in the rates of success of quit attempts. This difference in success rate is not attributable to differences in adoption of effective smoking cessation aids.

\section{BACKGROUND}

In England, cigarette smoking killed about 82,000 men and women in 2005 (17\% of all deaths of adults aged 35 years and over). ${ }^{1}$ The rate of decline in smoking prevalence has slowed considerably in recent years ${ }^{2}$ and remains above $20 \%{ }^{3}$. There is a large socio-economic gradient with $29 \%$ of adults in 'routine and manual' occupational groups currently smoking cigarettes compared with $15 \%$ in 'professional and managerial' groups. $^{3}$ This gradient is estimated to be responsible for half of socioeconomic differences in mortality in men aged 35-69 years in developed countries. ${ }^{4}$ The present paper examines how far the gradient can be explained in terms of attempts to stop smoking and/or use of effective aids to cessation. This has implications for strategies aimed at narrowing the gap in smoking prevalence.

The UK government has set a reduction in smoking prevalence as a high priority. ${ }^{5}$ Its target is to reduce the prevalence of cigarette smoking among adults in England to $21 \%$ or less by 2010 , with a reduction in prevalence among routine and manual groups to $26 \%$ or less ${ }^{6}$. Reducing the gap in smoking prevalence requires a better understanding of what is causing it. There is evidence that much of the socioeconomic difference in smoking prevalence is attributable to differences in cessation rates. For example, a pseudo-birth-cohort analysis with combined data from the 1972-2004/05 General Household Surveys showed that smokers born more recently (1956-1985) were less likely to give up smoking than earlier cohorts and that inequalities in smoking prevalence between the manual and non-manual group increased with age; the vast majority of manual men and women who started smoking remained smokers. ${ }^{7}$

What is less clear is what underlies the difference in cessation rates. The process of becoming an ex-smoker involves two potentially separable stages: initiating the quit attempt and then maintaining abstinence. Different factors may contribute to each of these. ${ }^{8,9}$ There is relatively little evidence on how far socio-economic status (SES) 
contributes to each of these phases. One study from the UK found no difference in attempts to stop by SES but a difference in success rates ${ }^{9}$ while another found no difference in attempts but those with an intermediate level of educational attainment were less likely than those with the lowest level to succeed ${ }^{8}$. However, both studies were small and the second study involved telephone interviews which may undermine representativeness.

We are aware of no other studies that have separated out attempts and success of attempts in this way. However, there is a large body of research on motivation to quit. One study found that smokers with low education and with low income were less likely to report the intention to quit than smokers with high education and income ${ }^{10}$ However, other, much larger studies of representative samples in the UK have consistently shown that smokers with lower SES report wanting to stop to the same degree as higher SES smokers. ${ }^{3,11}$ Social deprivation has been associated with "hard core" smoking; a syndrome which includes the absence of the desire to give up smoking ${ }^{12}$; however, this construct also includes indices of dependence and it is not clear how far the latter might account for the SES gradient. Taken together, these data suggest that there would be little or no difference in attempts to stop by SES. However, there may be many factors other than motivation that contribute to this behaviour.

It is also possible that SES differences in rates of becoming an ex-smoker are due to more deprived smokers being less likely to use effective aids to cessation such as nicotine replacement therapy (NRT) or the National Health Service (NHS) Stop Smoking Services. This is what would be predicted by the 'inverse care law' ${ }^{13}$ which states that individuals in lower strata of society who have the greatest need to healthcare services are least likely to gain benefit from them. We are aware of one study carried out in the US examining this in the case of smoking cessation. ${ }^{14}$ This study found that utilisation of behavioural and pharmacological aids to cessation was higher in more highly educated and more affluent smokers. The healthcare system in the US is different from the UK and it remains to be seen whether a similar pattern would be observed in this country. A study on utilisation of behavioural support, provided free of charge via the NHS in the UK, reported that smokers from more deprived geographical areas were more, rather than less, likely to use this service. ${ }^{15,16}$ However, we are not aware of good evidence from the UK on the social gradient in use of other aids such as NRT. A survey of utilisation of medications in the UK found no evidence for differences by SES but the sample was small and would have had limited power to detect a gradient. ${ }^{17}$

The present paper uses data from the 'Smoking Toolkit Study' (a series of monthly national surveys focusing on smoking and smoking cessation patterns), to examine 
socio-economic differences in: 1) attempts to stop smoking in the past year, 2) use of aids to cessation of those attempts, and 3) short-term 'success' rates.

\section{METHOD}

The Smoking Toolkit Study has been designed to provide ongoing, up-to-date national statistics on key performance indicators relating to smoking cessation to guide policy and clinical practice. ${ }^{18}$ The study involves monthly household surveys of national samples in England, carried out by the British Market Research Bureau.

The survey uses computer-assisted face to face interviews and a two-stage mixed probability and quota sampling method designed to maximise representativeness within the age range 16 and over. In the first stage, grouped output areas (containing 300 households) have an equal chance of being selected. The interviewers then go to the selected areas and attempt to secure interviews with members of households - one member per household, according to quotas based on known percentages for age, sex, social grade, region, working status, and presence of children in the population.

A total of 25,591 respondents were interviewed from November 2006 through to January 2008. Demographic information and smoking status were assessed by using standard questions based on those used in other national surveys. ${ }^{17}$ The data were weighted by age, sex, and socioeconomic group to match the 2001 Census.

People who had smoked in the past year were identified by asking respondents to indicate which of a series of statements applied to them: a) I smoke cigarettes (including hand-rolled) every day; b) I smoke cigarettes (including hand-rolled), but not every day; c) I do not smoke cigarettes at all, but I do smoke tobacco of some kind (e.g. pipe or cigar); d) I have stopped smoking completely in the last year; e) I stopped smoking completely more than a year ago; f) I have never been a smoker (i.e. smoked for a year or more). Cigarette smoking prevalence (using responses a. and b.) was calculated at $24 \%$ which is slightly higher than the 2006 data from the General Household Survey for England $(22 \%)^{3}$ and the 2007 Office of National Statistics omnibus survey for Great Britain $(22 \%)^{17}$. Those who endorsed a) to d) $(\mathrm{N}=6,950)$ comprised the sample.

The number of quit attempts in the last year was assessed by asking: "How many serious attempts to stop smoking have you made in the last 12 months? By serious attempt I mean you decided that you would try to make sure you never smoked again. Please include any attempt that you are currently making and please include any successful attempt made within the last year." 
The use of smoking cessation aids was assessed for the three most recent quit attempts and included: NRT over the counter (e.g. patches, gum, inhaler), NRT on prescription or given by a health professional, bupropion, varenicline, attending a NHS Stop Smoking Service group session or an NHS Stop Smoking Service individual counselling session.

Short-term success at stopping smoking was assessed by asking respondents who had made a quit attempt during the past 12 months whether they were still not smoking. This was checked against their response to the smoking status question (above).

Nicotine dependence in current smokers was assessed by the number of cigarettes smoked per day and the Fagerström Test for Nicotine Dependence (FTND) ${ }^{19}$. The sum score on the 6 items of the FTND has a range from $0-10$, with higher scores indicating higher levels of nicotine dependence.

Social grade was defined according to the National Statistics Socio-Economic Classification as: AB (managerial and professional occupations), C1 (intermediate occupations), C2 (small employers and own account workers), D (lower supervisory and technical occupations), and E (semi-routine and routine occupations, never workers, and long-term unemployed).

The statistical significance of differences in proportions across social grade was calculated using chisquared tests. The significance in cigarettes per day and FTND scores were assessed by means of analyses of variance. To examine social grade differences in proportions adjusting for age and sex we used multiple logistic regression analyses with all these predictors entered together.

\section{RESULTS}

The prevalence of smoking, including other forms of tobacco than cigarettes, was $25 \%$ with a large social gradient from $16 \%$ in $A B$ to $37 \%$ in $E(p<0.001$ by chisquared test). A similar gradient was found among smokers who had smoked in the past year for cigarettes per day and nicotine dependence. The mean number of cigarettes smoked per day ranged from $12.1(S D=8.7)$ in $A B$ to $15.8(S D=10.0)$ in $E$, and the mean level of nicotine dependence (according to the FTND) ranged from 2.3 $(S D=2.3)$ in $A B$ to $3.8(S D=2.6)$ in $E(p<0.001$ by analysis of variance in both cases) (table 1). 
There was no social gradient in attempts to stop smoking. Neither did we find a significantly lower rate of use of NRT bought over the counter or the NHS Stop Smoking Services in lower social grades (table 1). In fact, smokers from lower social grades were more likely to use NRT obtained on prescription than those in higher grades ( $p=0.016$ by chisquared test). There was also a small and non-significant gradient in use of NHS Stop Smoking Services. By contrast there was a large socioeconomic gradient in the success rates of those who attempted to stop with those in the lowest social grade having just over half the success rate of those in the highest $(p<0.001)$ (table 1 , figure 1$)$.

A multiple logistic regression analysis showed that the social gradient in cessation rate remained after adjusting for age and sex $(O R=0.86, p<0.001)$. Compared with the highest social grade $A B$, the odds of having stopped smoking were significantly lower the lower the social grades: $\mathrm{OR}=0.79(95 \% \mathrm{CI}=0.59,1.05)$ for social grade $\mathrm{C} 1$, $\mathrm{OR}=0.67(95 \% \mathrm{CI}=0.49,0.90)$ for social grade $\mathrm{C} 2, \mathrm{OR}=0.68(95 \% \mathrm{CI}=0.50,0.93)$ for social grade $D$, and $O R=0.50(0.33,0.74)$ for the lowest social grade $E$. The reverse social gradient in use of NRT obtained on prescription also remained after this adjustment $(\mathrm{OR}=1.11, \mathrm{p}=0.027)$.

\section{DISCUSSION}

Our results indicate that socio-economic differences in smoking cessation rates cannot be attributed to a lower likelihood of making quit attempts or using aids for smoking cessation. More deprived smokers appear to be engaged with public health messages about smoking, but their quit attempts are less likely to succeed.

Our finding of no social gradient in attempts to stop supports those of West et al. ${ }^{9}$ and Hyland et al. ${ }^{8}$. The finding of a social gradient in success of attempts is in accordance with West et al. ${ }^{9}$ but conflicts with Hyland et al. ${ }^{8}$. The size of the present sample and the strength of the association between social grade and success leads to the view that the association found here is genuine.

Our finding that lower SES smokers were at least as likely to use aids to cessation conflicts with those of Shiffman et al. ${ }^{14}$ One possible explanation for this may be that treatments for smoking cessation are reimbursed in England but not in the USA. As a consequence, increased use of pharmacological treatment among US smokers with higher incomes may be due to greater insurance coverage or greater ability to pay for non-covered medications. ${ }^{14}$ However, we did not find a social gradient in NRT purchased over-the-counter either. This suggests that another factor must be in play. The UK government has adopted a strategy of maximising NRT use in the smoking population and this may have played a role. However, there may be other cultural 
and economic factors involved as well, such as the fact that the US has a wider income gradient than the UK..$^{20}$

The question remains as to what factors may account for differences in quitting success by SES. Nicotine dependence is one possibility. Our data confirm previous reports of higher nicotine dependence scores in smokers from more deprived socioeconomic groups ${ }^{12}$, and nicotine dependence has been found in other studies to predict failure of attempts to stop smoking. ${ }^{21}$ Another possible explanation is that smokers in more deprived socio-economic groups have more smokers in their immediate circle of family, co-workers and friends. Qualitative research has shown that smoking is often deeply embedded in the lives of disadvantaged smokers because they live, socialize, and/or work with other smokers, which makes it more difficult to refrain from smoking. ${ }^{22}$ It has also been shown that smoking cessation is influenced by unfavourable social trajectories such as childhood and educational disadvantages and early motherhood in female smokers. ${ }^{23}$ Smokers from more deprived socioeconomic groups also have higher levels of stress which can play a role in relapse because smokers often use smoking to cope with stressful aspects of their lives. ${ }^{22,24}$ Factors underlying differences in quitting success by SES is an important topic for future research.

One limitation of the current study is that it uses cross-sectional data. Many of the respondents who were not smoking at the time of the survey will have relapsed afterwards. Further research using longitudinal data is needed to establish the differential long-term success rates of smokers in different socio-economic groups. Secondly, we only measured the smokers' reports of having used or not used a certain treatment for smoking cessation and not how compliant they were with the use of that treatment (e.g. if they used the prescribed dose of NRT or to which extent they attended behavioural counselling sessions). It cannot be ruled out that compliance with treatment varies across socio-economic groups. A third potential limitation is the fact that our study did not involve a pure probability sample. However, the present method has been found to yield results very similar to those of pure probability samples and neither of these can eliminate the problem of nonresponse bias. The major limitation of the study concerns the inability to test other hypotheses to explain the social gradient in success rates. Because the data were cross-sectional we could not reliably assess the role of factors such as nicotine dependence and social milieu that might be subject to recall bias in those who had attained abstinence. This should be a priority for future studies.

We conclude that smokers from more deprived socio-economic groups are less successful in quitting smoking but try to quit as often and use aids to cessation at least as often as less deprived smokers. Research now needs to focus on possible 
explanations for this so that this important source of health inequalities can be addressed. 


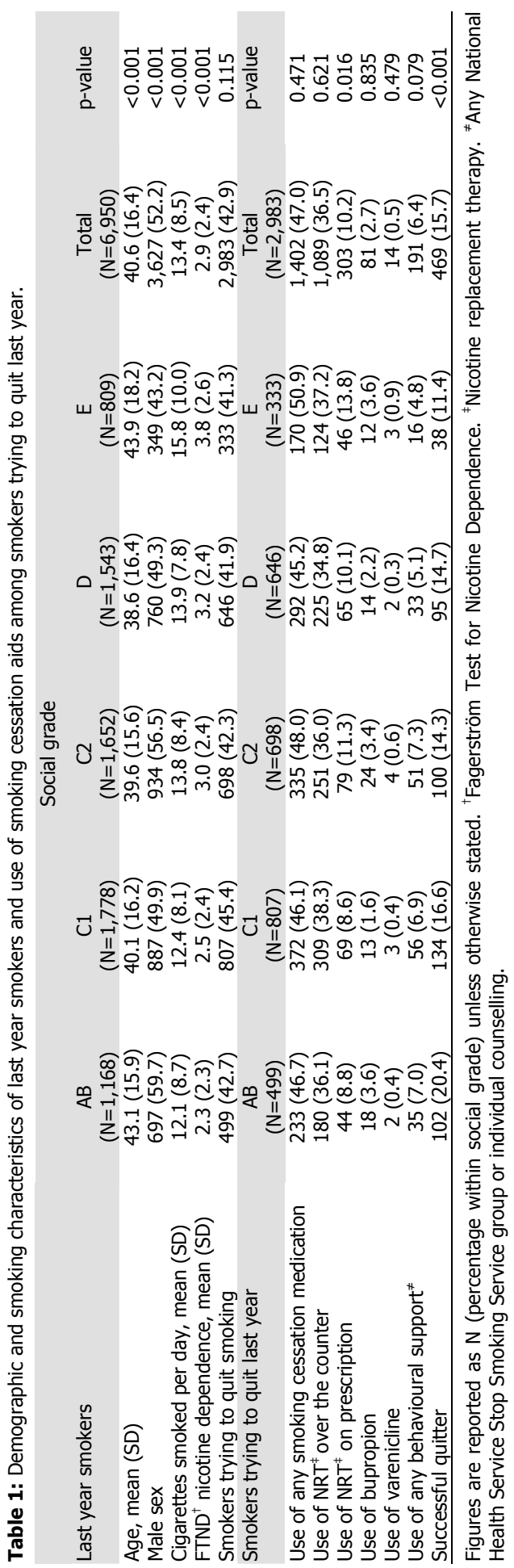



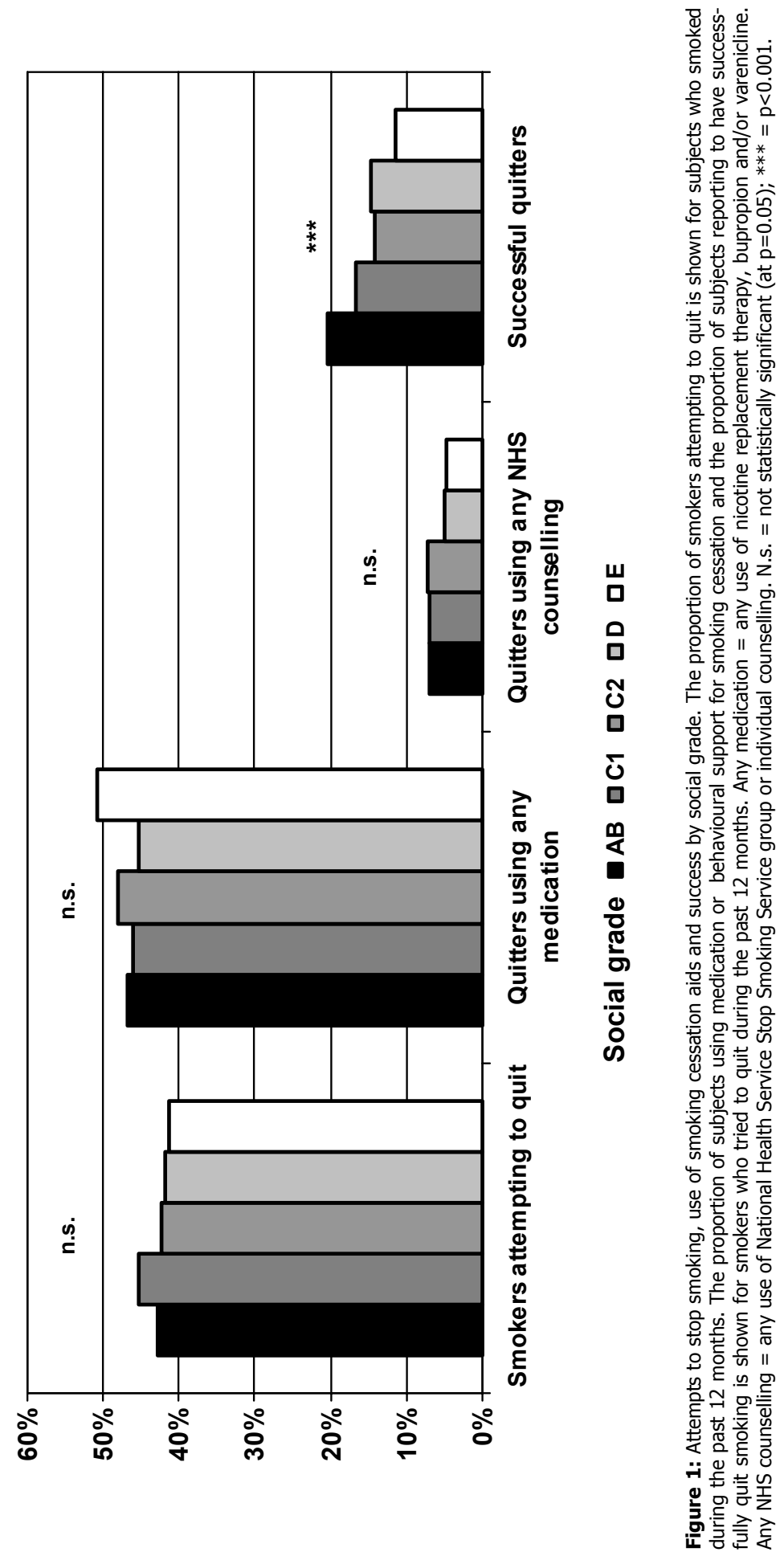


\section{REFERENCES}

1 The Information Centre. Statistics on Smoking: England, 2007. www.ic.nhs.uk

2 Jarvis MJ. Monitoring cigarette smoking prevalence in Britain in a timely fashion. Addiction 2003; 98:1569-1574

3 Goddard E. General Household Survey 2006. Smoking and drinking among adults, 2006. London: Office for National Statistics, 2007

4 Jha $\mathrm{P}$, Peto R, Zatonski W, et al. Social inequalities in male mortality, and in male mortality from smoking: indirect estimation from national death rates in England and Wales, Poland, and North America. Lancet 2006; 368:367-370

5 Department of Health. Smoking kills: a White Paper on tobacco. London: The Stationary Office, 1998

6 Department of Health. The NHS Cancer Plan: www.doh.gov.uk/cancer/cancerplan.htm, 2000

7 Davy M. Socio-economic inequalities in smoking: an examination of generational trends in Great Britain. Health Stat Q 2007:26-34

8 Hyland A, Borland R, Li Q, et al. Individual-level predictors of cessation behaviours among participants in the International Tobacco Control (ITC) Four Country Survey. Tob Control 2006; 15 Suppl 3:iii83-94

9 West R, McEwen A, Bolling K, et al. Smoking cessation and smoking patterns in the general population: a 1-year follow-up. Addiction 2001; 96:891-902

10 Siahpush M, McNeill A, Borland R, et al. Socioeconomic variations in nicotine dependence, self-efficacy, and intention to quit across four countries: findings from the International Tobacco Control (ITC) Four Country Survey. Tob Control 2006; 15 Suppl 3:iii71-75

11 Goddard E. General Household Survey 2005. Smoking and drinking among adults, 2005. London: Office for National Statistics, 2006

12 Jarvis MJ, Wardle J, Waller J, et al. Prevalence of hardcore smoking in England, and associated attitudes and beliefs: cross sectional study. BMJ 2003; 326:1061

13 Hart JT. The inverse care law. Lancet $1971 ; 1: 405-412$

14 Shiffman S, Brockwell SE, Pillitteri JL, et al. Individual differences in adoption of treatment for smoking cessation: demographic and smoking history characteristics. Drug Alcohol Depend 2008; 93:121-131

15 Bauld L, Judge K, Platt S. Assessing the impact of smoking cessation services on reducing health inequalities in England: observational study. Tob Control 2007; 16:400-404

16 Chesterman J, Judge $\mathrm{K}$, Bauld L, et al. How effective are the English smoking treatment services in reaching disadvantaged smokers? Addiction 2005; 100 Suppl 2:36-45

17 Lader D. Smoking related behaviour and attitudes, 2007. A report on research using the National Statistics Omnibus Survey produced on behalf of the NHS Information Centre for health and social care. London: Office for National Statistics, 2008

18 West R. The Smoking Toolkit Study: www.smokinginengland.info, 2008

19 Heatherton TF, Kozlowski LT, Frecker RC, et al. The Fagerström Test for Nicotine Dependence: a revision of the Fagerström Tolerance Questionnaire. Br J Addict 1991; 86:1119-1127

20 Central Intelligence Agency. The World Fact Book 2005. http://www.cia.gov/cia/publications

21 West R. Defining and assessing nicotine dependence in humans. In: Bock G, Goode J, eds. Understanding Nicotine and Tobacco Addiction. London: Wiley, 2006

22 Wiltshire S, Bancroft A, Parry O, et al. 'I came back here and started smoking again': perceptions and experiences of quitting among disadvantaged smokers. Health Educ Res 2003; 18:292-303

23 Graham H, Inskip HM, Francis B, et al. Pathways of disadvantage and smoking careers: evidence and policy implications. J Epidemiol Community Health 2006; 60 Suppl 2:7-12

24 Siahpush M, Carlin JB. Financial stress, smoking cessation and relapse: results from a prospective study of an Australian national sample. Addiction 2006; 101:121-127 


\section{Chapter 11}

General discussion 


\section{MAIN CONCLUSION FROM THE TRIAL}

In the main part of this dissertation, we presented results from a randomized controlled trial on the efficacy of "confrontational counselling" for smoking cessation in smokers motivated to quit and with previously undetected COPD. We hypothesised that confronting smokers with the results from spirometry may be effective when it is integrated into state-of-the-art smoking cessation treatment. Our confrontational counselling approach consisted of several counselling sessions on an individual, faceto-face level, under supervision of a trained respiratory nurse, and in combination with evidence-based pharmacological treatment (nortriptyline) for smoking cessation.

Our results clearly show that confrontational counselling did not increase the longterm (12-month) prolonged quit rate compared with an equally intensive treatment in which smokers were not confronted with their abnormal spirometry. The stunning relapse rates of $88 \%$ and higher, one year after the target quit date, dramatically emphasize the difficulty tobacco addicted smokers experience with quitting smoking and highlight the need for treating tobacco addiction as a chronic relapsing disorder. Even in smokers who are highly motivated to stop smoking, who have an urgent need to quit due to their respiratory symptoms, and who receive individual support in combination with pharmacotherapy, only a small fraction remains long-term abstinent.

The results from our mediation analysis show that smoking-related cognitions can be influenced by confrontational counselling. In smokers with previously undetected COPD, confrontational counselling increases risk perceptions and self-efficacy, and decreases self-exempting beliefs (i.e. risk denial). These changes in mediators are associated with a higher likelihood of abstinence from smoking until shortly after the treatment. This effect, however, diminished during the follow-up period in which the participants did not receive any more counselling.

The results from our ethical analysis show that labelling with COPD - confronting smokers with a previously undetected or unrecognized disease status - is probably a less important issue in the discussion about the pros and cons of early detection of COPD. The vast majority of the participants from our trial, irrespectively of the intervention received and treatment outcome, agreed that it is justified to measure lung function in heavy smokers, because it raises consciousness about the negative effects of smoking, helps to prevent disease, or increases motivation to stop smoking. 


\section{WHAT'S NEXT?}

It is remarkable that the most lethal consumer product ever produced can still be legally sold: cigarettes. Marlboro, the world's best selling cigarette, celebrated its $50^{\text {th }}$ anniversary in November 2005. In the United States of America alone, Marlboro cigarettes have killed more than 2.3 million Americans since 1955 with another expected 1.6 million deaths in the next 10 years. ${ }^{1}$ In February 2005, the World Health Organization Framework Convention on Tobacco Control (WHO FCTC) came into force. ${ }^{2}$ The objective of the treaty, that is legally binding in 154 ratifying countries, is "to protect present and future generations from the devastating health, social, environmental and economic consequences of tobacco consumption and exposure to tobacco smoke. ${ }^{\prime 2}$ The treaty provides a framework of tobacco control measures, including price and tax measures to reduce the demand for tobacco, packaging and labelling measures to inform users about the harmful effects of tobacco use, a ban on tobacco advertising, promotion and sponsorship, and measures of protection from tobacco smoke exposure. Concerning the latter, the Dutch government has finally decided to introduce a smoking ban in pubs and restaurants by 1 July 2008. It is the goal of the Ministry of Health, Welfare and Sport (VWS) to reduce the prevalence of smokers to $20 \%$ in $2010 .^{3}$ This is a very ambitious goal. For as long as cigarettes can be legally marketed, there will be smokers, smokers who will get addicted to nicotine and tobacco, and who will need help to quit smoking. There are necessarily two implications for the health care system to help addicted smokers to quit; (1) to increase the efficacy of smoking cessation therapies, and (2) to increase the accessibility and use of these therapies.

\section{Increasing the efficacy of smoking cessation treatments}

With regard to pharmacotherapy, alternative smoking cessation drugs have been developed or are under development. Varenicline, a a4 32 nicotine acetylcholine receptor partial agonist, has been designed to reduce tobacco withdrawal symptoms and the reinforcing effects of smoking. It has been shown to be safe, well-tolerated, and more effective than bupropion and placebo. ${ }^{4,5}$ Rimonabant, a cannaboid-1 $\left(\mathrm{CB}_{1}\right)$ receptor antagonist, may be effective in smoking cessation ${ }^{6}$, especially because it has the potential to limit post-cessation weight gain - a significant barrier to cessation for many smokers. A different promising approach, especially for relapse prevention, is vaccination against nicotine. The rationale is to induce antibodies which bind nicotine in the blood and thereby preventing nicotine from crossing the blood-brain barrier. Three vaccines are currently under development, named NicQb ${ }^{7}$, $\mathrm{TA}^{-\mathrm{NIC}^{8}}$, and NicVax ${ }^{9}$.

Compared with pharmacological research, much less effort has been put into research on behavioural treatment of tobacco use. Developing more effective behavioural treatments (and combination treatments with pharmacotherapy) is an impor- 
tant area of future research. One major issue in this respect is optimizing the content and dose of treatments. Cognitive behavioural therapy for smoking cessation, for example, is a potentially effective approach that could be further advanced. The results from our trial show that cognitions related with smoking can be influenced in a way to increase smoking cessation. However, much more research is needed on the underlying cognitive mechanisms of nicotine and tobacco addiction and how to intervene in these. Furthermore, whereas research shows a general dose-response relationship between treatment intensity and success, ${ }_{1}^{10}$ we do not know much about the optimal dose of specific behavioural treatments (in terms of number and duration of sessions) and the timing of treatment sessions.

Another major issue with regard to the efficacy of behavioural interventions is tailoring of interventions to different types of smokers. We start to recognize that the efficacy and effectiveness of various pharmacological aids for smoking cessation, with their diverse mechanisms of actions, differ in various types of smokers. For example, the anti-depressants bupropion and nortriptyline may be more effective than nicotine replacement therapy in smokers with psychiatric co-morbidity ${ }^{10}$, and rimonabant may be more useful in smokers who fear post-cessation weight gain. ${ }^{11}$ First studies on the concepts of negative and positive reinforcement show that smokers who predominantly smoke for positive reinforcement (smoke for pleasure) respond better to varenicline than smokers who smoke for negative reinforcement (to cope with withdrawal symptoms). ${ }^{12}$ It is not unlikely to assume that various types of behavioural treatments could also be tailored to specific groups of smokers. For example, confronting smokers with a biomarker for a smoking-related disease, such as spirometry for COPD, may be effective in smokers who are interested in their (respiratory) health and who are therefore susceptible to related health warnings, but this confrontation may be counterproductive in smokers with no such health perception (as we concluded from our trial). If we were able to identify characteristics of smokers that are associated with the outcomes of specific behavioural treatment alternatives, we could tailor smoking treatment to the individual smoker's needs and thereby increase the efficacy (and cost-effectiveness) of smoking cessation therapies. This includes, for example, offering less intensive treatment (brief advice) to smokers with a profile that is associated with a higher chance of quitting, and more intensive treatment (several weeks or months of counselling) to smokers who have a more urgent need but find it more difficult to quit, such as COPD patients.

\section{Increasing the accessibility and use of smoking cessation therapies}

Smoking cessation is one of the most effective measures to improve health, and behavioural and pharmacological treatments can double a smoker's chance of quitting smoking. Nevertheless, smoking cessation treatments remain underused. Data from the 2000 US National Health Interview Survey suggest that only $22 \%$ of smok- 
ers trying to quit used any pharmacological treatment, and not more than $1 \%$ used behavioural counselling. ${ }^{13}$ Data from the 2006 UK National Statistics Omnibus Survey indicate that only $28 \%$ of smokers used a pharmacological treatment in an attempt to quit smoking, $17 \%$ asked a doctor or other health professional for help, and only $10 \%$ joined a stop-smoking group. ${ }^{14}$ Efforts are needed to promote the use and to minimize the barriers that prevent the adoption of evidence-based smoking cessation therapies.

One such strategy is the reimbursement of smoking cessation aids. The costs of smoking cessation aids are a significant barrier for many smokers. Reimbursement of smoking cessation aids can lead to a two- to threefold increase of the use of pharmacotherapy to aid a quit attempt. ${ }^{15}$ This has also been demonstrated in Dutch research several years ago, ${ }^{16}$ but has not led to the reimbursement of smoking cessation aids in the Netherlands ever since, though there currently is a discussion to do so. ${ }^{17}$ Another strategy to increase the accessibility and use of smoking cessation therapies is to develop a national infrastructure of smoking cessation services. A good example is the UK National Health Service (NHS) Stop Smoking Service. ${ }^{18}$ The NHS offers free local support (one-to-one or group counselling) to smokers who want to quit smoking. Further development of an easily accessible, nationwide service for smoking cessation in the Netherlands that offers free behavioural treatment under supervision of health care professionals specialised in smoking cessation treatment and in combination with free pharmacological treatment would be an important contribution to the reduction and smoking prevalence and related morbidity and mortality.

\section{FINAL REMARK}

People who are against the reimbursement of pharmacological and behavioural treatment for smoking cessation argue that smoking is a life-style behaviour and that smokers have to take responsibility for their own behaviour. This may be true for the uptake of smoking but is certainly not true for stopping smoking because of the addictive nature of nicotine and tobacco. People who do not want to put money in smoking cessation therapies and the research to improve these therapies should consider the fact that smoking is the major risk factor for the onset and the progression of many highly prevalent diseases. Investing in (research on) smoking cessation therapies today is likely to result in a decrease of smoking-related burden of disease and an increase of quality of life tomorrow. Whether our society is willing to pay for this is a question that remains to be answered. 


\section{REFERENCES}

1 Hyland A, Goldstein R, Brown A, et al. Happy Birthday Marlboro: the cigarette whose taste outlasts its customers. Tob Control 2006; 15:75-77

2 World Health Organisation (WHO). WHO Framework on Tobacco Control. Geneva: WHO, 2005

3 Ministerie voor Volksgezondheid Welzijn en Sport (VWS) [Ministry of Health Welfare and Sport]. Nationaal programma tabaksontmoediging. [National program for tobacco discouragement.]. The Hague: VWS, 2006

4 Gonzales D, Rennard SI, Nides M, et al. Varenicline, an \{alpha\}4beta2 Nicotinic Acetylcholine Receptor Partial Agonist, vs Sustained-Release Bupropion and Placebo for Smoking Cessation: A Randomized Controlled Trial. JAMA 2006; 296:47-55

5 Jorenby DE, Hays JT, Rigotti NA, et al. Efficacy of Varenicline, an \{alpha\}4beta2 Nicotinic Acetylcholine Receptor Partial Agonist, vs Placebo or Sustained-Release Bupropion for Smoking Cessation: A Randomized Controlled Trial. JAMA 2006; 296:56-63

6 Cahill K, Ussher M. Cannabinoid type 1 receptor antagonists (rimonabant) for smoking cessation. Cochrane Database Syst Rev 2007

7 Maurer P, Jennings GT, Willers J, et al. A therapeutic vaccine for nicotine dependence: preclinical efficacy, and Phase I safety and immunogenicity. Eur J Immunol 2005; 35:2031-2040

8 Trial watch: Xenova's TA-NIC vaccine shows promise. Expert Rev Vaccines 2004; 3:386

9 Hatsukami DK, Rennard S, Jorenby D, et al. Safety and immunogenicity of a nicotine conjugate vaccine in current smokers. Clinical Pharmacology \& Therapeutics 2005; 78:456-467

10 Fiore MC, Bailey WC, Cohen SJ, et al. Treating tobacco use and dependence. Clinical Practical Guideline. Rockville, MD: US Department of Health and Human Services, 2000

11 Fagerström K, Balfour DJ. Neuropharmacology and potential efficacy of new treatments for tobacco dependence. Expert Opin Investig Drugs 2006; 15:107-116

12 Fagerström K, Jimenez-Ruiz CA, Astray Mochales J, et al. Can smoking for positive or negative reinforcement together with dependence help us better diagnose smokers? Journal of Smoking Cessation 2007; 2:5-7

13 Cokkinides $V E$, Ward $E$, Jemal $A$, et al. Under-use of smoking-cessation treatments: results from the National Health Interview Survey, 2000. Am J Prev Med 2005; 28:119-122

14 Lader D. Smoking related behaviour and attitudes, 2006. A report on research using the National Statistics Omnibus Survey produced on behalf of the Information Centre for health and social care. London: Office for National Statistics, 2007

15 Kaper J, Wagena EJ, Severens JL, et al. Healthcare financing systems for increasing the use of tobacco dependence treatment. Cochrane Database Syst Rev 2005

16 Kaper J, Wagena EJ, Willemsen MC, et al. Reimbursement for smoking cessation treatment may double the abstinence rate: results of a randomized trial. Addiction 2005; 100:1012-1020

17 Van Rootselaar F. De laatste sigaret. [The last cigarette.]. CVZ Magazine, Winter 2007/2008

18 UK Deparment for Health. http://gosmokefree.nhs.uk/, accessed 20 May 2008 


\section{Summary}


Chronic obstructive pulmonary disease (COPD) is a preventable and treatable disease which is characterized by airflow limitation that is not fully reversible. It is the fifth leading cause of death worldwide, and projections for 2020 indicate a further increase in global mortality, eventually placing COPD in third position of lethal diseases. Cigarette smoking is by far the most important risk factor for COPD; $15 \%$ to $50 \%$ of smokers are estimated to develop COPD during their lifetime. Smoking cessation is the single most effective way to reduce the risk of developing COPD and to affect the outcome in patients at all stages of the disease.

Despite the well-known health risks, many people still smoke (in the Netherlands currently about $29 \%$ ). Whereas most smokers do want to quit, only a fraction of those who try remain long-term abstinent, mainly because of nicotine and tobacco dependence. The two main types of treatment that can help addicted smokers to quit are pharmacological and behavioural treatment. But even when treated with a combination of both, about $75 \%$ or more of smokers making an attempt to quit relapse within the first year and resume smoking.

It is important to improve smoking cessation strategies, especially in smokers with COPD, who have a greater and more urgent need to stop smoking than the average smoker. A potentially successful motivational tool may be spirometry, the gold standard for the diagnosis and assessment of COPD. Confronting smokers with an objectively identified negative consequence of smoking by spirometry (airflow limitation, COPD) may possibly improve the outcome of their attempt to quit. Various studies have been performed to study the efficacy of spirometry for smoking cessation but the results are inconclusive.

The main part of this thesis is a randomized controlled trial on the efficacy of COnfronting SMOkers with spirometry for smoking cessation; the COSMO trial (trial register: Netherlands Trial Register ISRCTN 64481813). We hypothesised that early detection of COPD and confrontation with spirometry for smoking cessation may be effective if certain conditions are met which we describe in chapter 4; confronting patients with COPD should not be an isolated approach but should be integrated into state-of-the-art smoking cessation treatment consisting of several counselling sessions on an individual, face-to-face level, under supervision of a trained smoking cessation specialist, and in combination with evidence-based pharmacotherapy for smoking cessation. We termed this approach "confrontational counselling", and tested its efficacy in a randomized controlled trial. The protocol of the COSMO trial is presented in chapter $\mathbf{5}$.

All smokers from the COSMO trial had previously undetected airflow limitation. It has been suggested that early detection of smokers with COPD may be enhanced by using questionnaires to identify patients with a high likelihood of having airflow 
limitation before using spirometry. GPs may use a COPD diagnostic questionnaire that is recommended by the International Primary Care Respiratory Group. Before this new diagnostic questionnaire can be accepted and applied reliably in clinical practice, the analysis of the underlying model must be repeated on new data collected from an appropriate sample of subjects from a different setting. We performed this external validation in 676 smokers that we screened for eligibility for participation in the COSMO trial (chapter 6). The ability of the COPD diagnostic questionnaire to discriminate between subjects with and without COPD was poor: the area under the receiver operating characteristics curve $\left(R O C_{A U C}\right)$ was 0.65 (the $R O C_{A U C}$ can take values from 0.5 (totally uninformative) to 1.0 (totally informative diagnostic test)). We concluded that this questionnaire is probably not useful as a diagnostic tool to identify patients with an increased risk of airflow limitation in a population of current heavy smokers.

The primary research question of the COSMO trial was whether confrontational counselling increased long-term abstinence rates from smoking during a period of 52 weeks after the target quit date (chapter 7). A total of 296 smokers with previously undetected mild to moderate airflow limitation were randomly allocated to; medium intensity confrontational counselling delivered by a respiratory nurse combined with nortriptyline for smoking cessation (experimental group), medium intensity health education delivered by a respiratory nurse combined with nortriptyline for smoking cessation (control group 1), or "care as usual" for smoking cessation by the general practitioner (control group 2). Only smokers from the experimental group were confronted with their abnormal lung function. The results showed no difference in cotinine validated prolonged abstinence rate between the experimental group and control group 1 from week 5 through 52 (odds ratio $(\mathrm{OR})=0.96,95 \%$ confidence interval $(\mathrm{CI})=0.43,2.18)$. The high failure rates ( $88 \%$ and higher) highlight the need for treating tobacco addiction as a chronic relapsing disorder, especially in smokers with respiratory disease, who have a more urgent need to stop.

We were not only interested whether or not confrontational counselling was effective but also how it might work. When we designed the intervention, we hypothesised that confrontational counselling - through labelling with a potentially life-threatening illness status - increases risk perception and health concerns and decreases selfexempting beliefs in smokers, changes which in turn may account for abstinence from smoking. To analyse these mechanisms of change, we performed a mediation analysis on the effects of confrontational counselling on short-term abstinence from smoking in the 228 smokers from the experimental group and control group 1 of the COSMO trial (chapter 8). Cotinine validated abstinence rates from smoking at 5 weeks after the target quit date were $43.1 \%$ in the experimental group versus $31.3 \%$ in control group $1(\mathrm{OR}=1.67,95 \% \mathrm{CI}=0.97,2.87)$. The effect of confrontational counselling on abstinence was independently mediated by the expectation of 
getting a serious smoking related disease in the future $(\mathrm{OR}=1.76,95 \% \mathrm{CI}=1.03$, $3.00)$, self-exempting beliefs $(\mathrm{OR}=0.42,95 \% \mathrm{CI}=0.21,0.84)$, and self-efficacy $(\mathrm{OR}=1.38,95 \% \mathrm{CI}=1.11,1.73)$.

Irrespectively of its efficacy, it is worth considering whether an approach of early detection and labelling with COPD is justifiable. Labelling with disease - confronting subjects with a previously undetected or unrecognized disease status - has long been an issue of debate in the medical field. In most cases, the debate has focused on the potential adverse effects of labelling, but it is also possible that it has positive effects (e.g., labelling with COPD may motivate smokers to quit smoking). We conducted semi-structured ethical exit interviews on this subject in 205 participants from the COSMO trial to assess their opinions on the effectiveness of spirometry for smoking cessation, the justification of early detection of airflow limitation in smokers, and the impact of confrontation with COPD (chapter 9). Our results showed that labelling with disease is probably a less important issue in the discussion about the pros and cons of early detection of COPD; the majority of participants (86\%) agreed that it is justified to measure lung function in heavy smokers. These participants argued that measuring lung function raises consciousness about the negative effects of smoking, helps to prevent disease, or increases motivation to stop smoking. Most of the participants who disagreed argued that routinely measuring lung function in smokers would interfere with one's freedom of choice.

Apart from the COSMO trial, we conducted observational research on smoking cessation practices of Dutch health care providers. According to national and international guidelines on the treatment of tobacco dependence, health care providers such as GPs, cardiologists, lung physicians, and respiratory nurses can play an important role in reducing the prevalence of smoking. However, it is unclear what their smoking cessation practices, attitudes, and perceived effectiveness are. To answer these questions, we conducted national surveys among 834 GPs, 300 cardiologists, 258 lung physicians (these three surveys are combined in chapter 2), and 254 respiratory nurses (chapter 3). Results from theses surveys showed that the prevalence of current smoking among Dutch physicians and respiratory nurses has decreased sharply in recent years and is low (4-8\%) compared with the general population (about 29\%). Of the pharmacological aids for smoking cessation, physicians recommended bupropion most frequently to their patients, followed by nicotine patches and nicotine gum. Furthermore, more lung physicians than GPs and cardiologists recommended the use of bupropion, nicotine patch, and nicotine gum. The respiratory nurses reported to be compliant with the Dutch protocol for the treatment of nicotine and tobacco addiction (the so-called "L-MIS"); seven out of ten behavioural techniques for smoking cessation from the protocol were used by more than $94 \%$ of the respondents. Perceived lack of patient's motivation formed the most important threat to respiratory nurses' future smoking cessation activities. International guide- 
lines acknowledge that respiratory patients have a more urgent need to stop smoking but have more difficulty doing so. They should be offered the most intensive smoking cessation counselling in combination with pharmacotherapy. Behavioural counselling for smoking cessation may be more feasible for respiratory nurses than for physicians who often lack time.

As stated before, a considerable proportion of the population smokes despite the well-known health risks. Furthermore, there is a large socio-economic gradient in smoking in the Netherlands as in other developed countries with the highest prevalence of smoking in half-skilled and unskilled manual labour (44\% in the Netherlands in 2007) and the lowest prevalence in highly-skilled white-collar workers (27\%). This gradient is estimated to be responsible for half of socio-economic differences in mortality in men aged 35-69 years in developed countries. An important future goal is to reduce social health inequalities that are caused by smoking. Reducing inequalities in smoking prevalence requires a better understanding of what is causing it. We used data from 6,950 respondents to a UK cross-sectional household survey to examine the variation across social grades in: rates of attempts to stop smoking; use of pharmacological and behavioural treatment for smoking cessation; and success rates of quit attempts (chapter 10). A total of 2,983 respondents had tried to stop in the past year and 469 reported having stopped at the time of the survey. The results showed no difference across social grades in the rate of attempts to stop ( $42.7 \%$ in the highest social grade $A B$ to $41.3 \%$ in the lowest social grade $E$ ), use of medications to aid cessation (46.7\% of those making quit attempts in $A B$ to $50.9 \%$ in E) or use of National Health Service Stop Smoking Services (7.0\% of those making quit attempts in $A B$ to $4.8 \%$ in $E$ ). There was, however, a large difference in success rates: $20.4 \%$ in social grade $A B$ versus $11.4 \%$ in social grade $E$ of those who made attempts were still not smoking by the time of the survey. 



\section{Samenvatting}


Chronic obstructive pulmonary disease (COPD) is een aandoening die gekenmerkt wordt door een niet volledig reversibele luchtwegobstructie. COPD staat momenteel op de vijfde plaats van de belangrijkste doodsoorzaken wereldwijd, en er wordt een verdere toename voorspeld in mortaliteit wereldwijd waardoor COPD in 2020 de derde plaats in zou nemen. Het roken van sigaretten is op afstand de meest belangrijke risicofactor van COPD; naar schatting zal $15 \%$ tot $50 \%$ van alle rokers COPD ontwikkelen in de loop van hun leven. Stoppen-met-roken is de meest effectieve manier om het risico op ontstaan van COPD te verminderen en om de prognose te verbeteren bij alle patiënten, ongeacht de ernst van de aandoening.

Er zijn nog veel mensen die roken, ondanks de bekende gezondheidsrisico's van roken (in Nederland momenteel ongeveer 29\%). Het merendeel van de rokers wil wel stoppen met roken maar slechts een klein gedeelte lukt het om langdurig rookvrij te blijven. Dit komt voornamelijk door verslaving aan nicotine en tabak. De twee belangrijkste behandelingen die bestaan om verslaafde rokers bij het stoppen te helpen zijn farmacologische en gedragsmatige ondersteuning. Maar zelfs na een combinatietherapie begint minimaal $75 \%$ van de rokers die een stoppoging ondernemen binnen een jaar weer te roken.

Het is daarom belangrijk om bestaande stoppen-met-roken strategieën te verbeteren, vooral bij rokers met COPD omdat deze een dringendere behoefte hebben om te stoppen dan de gemiddelde roker. Spirometrie is de gouden standaard voor de diagnose van COPD en zou een geschikt middel kunnen zijn om de motivatie voor het stoppen-met-roken te vergroten. Rokers confronteren met de objectief vastgestelde negatieve gevolgen van roken (luchtwegobstructie, COPD) zou de uitkomst van hun stoppoging positief kunnen beïnvloeden. Een aantal studies is in het verleden uitgevoerd om het effect van spirometrie op stoppen-met-roken te bepalen, maar de resultaten zijn niet eenduidig.

Het hoofddeel van dit proefschrift is een gerandomiseerde gecontroleerde studie naar het effect van het confronteren van rokers met spirometrie om te stoppen met roken; de COSMO studie ("COnfronting SMOkers with spirometry for smoking cessation" - trial register: Netherlands Trial Register ISRCTN 64481813). Onze hypothese was dat vroegtijdig opsporen van COPD en confrontatie met spirometrie om te stoppen met roken effectief zou kunnen zijn indien aan bepaalde voorwaarden wordt voldaan. Deze voorwaarden beschrijven we in hoofdstuk 4; het confronteren van patiënten met COPD zou geen geïsoleerde aanpak moeten zijn maar geïntegreerd moeten worden in een state-of-the-art stoppen-met-roken behandeling die bestaat uit herhaalde individuele counseling sessies, onder begeleiding van een getrainde stoppen-met-roken specialist en in combinatie met aangetoond effectieve farmacotherapie voor stoppen-met-roken. We hebben deze aanpak "confronterende counse- 
ling" genoemd en getest in een experimentele studie. Het protocol van de COSMO studie wordt gepresenteerd in hoofdstuk 5.

Alle rokers die meededen aan de COSMO studie hadden niet eerder vastgestelde luchtwegobstructie. Het vroegtijdig diagnosticeren van rokers met COPD zou verbeterd kunnen worden door het gebruik van vragenlijsten voor het opsporen van patiënten met een hoger risico op luchtwegobstructie, voorafgaand aan spirometrie. Voor huisartsen bestaat een diagnostische vragenlijst die aanbevolen wordt door de International Primary Care Respiratory Group (een internationale vereniging van experts op het gebied van luchtwegklachten in de eerste lijn). Voordat een nieuw diagnostisch instrument gebruikt kan worden in de dagelijkse praktijk moet de analyse van het onderliggende model herhaald worden op nieuwe data die verzameld zijn in een bruikbare steekproef van mensen uit een andere setting. We voerden deze externe validering uit in 676 rokers die we hadden gescreend voor deelname aan de COSMO studie (hoofdstuk 6). Het vermogen van de diagnostische vragenlijst om onderscheid te maken tussen mensen met en zonder COPD was slecht: de "area under the receiver operating characteristics curve (ROCAUC)" was 0,65 (de ROCAUC kan een waarde aannemen tussen 0,5 (volledig onbruikbare diagnostische test) tot 1,0 (volledig informatieve test)). We concludeerden dat deze vragenlijst waarschijnlijk niet bruikbaar is als diagnostisch instrument voor het opsporen van patiënten met een verhoogd risico op luchtwegobstructie in een populatie van huidige rokers.

De primaire vraagstelling van de COSMO studie was of confronterende counseling de lange termijn abstinentie van roken vergroot gedurende een periode van 52 weken na de geplande stopdatum (hoofdstuk 7). In totaal werden 296 rokers met niet eerder vastgestelde lichte tot matige luchtwegobstructie op basis van toeval toegewezen aan: matig intensieve confronterende counseling door een longverpleegkundige gecombineerd met nortriptyline voor stoppen-met-roken (experimentele groep), matig intensieve gezondheidsvoorlichting door een longverpleegkundige gecombineerd met nortriptyline voor stoppen-met-roken (controlegroep 1), of gebruikelijke zorg voor stoppen-met-roken door de huisarts (controlegroep 2). Alleen rokers in de experimentele groep werden geconfronteerd met hun abnormale longfunctie. De resultaten lieten geen verschil zien in het percentage gestopte rokers tussen de experimentele groep en controlegroep 1 in de peridoe van week 5 tot week 52 (odds ratio $(\mathrm{OR})=0,96,95 \%$ betrouwbaarheidsinterval $(\mathrm{BI})=0,43-2,18)$. De bevinding dat een heel groot deel van de rokers niet erin slaagde om te stoppen met roken ( $88 \%$ en hoger) benadrukt de noodzaak om tabaksverslaving te behandelen als een chronische stoornis die gekenmerkt wordt door veelvuldige terugval. Een dergelijke, intensievere aanpak is vooral noodzakelijk voor rokers met een door roken veroorzaakte of verergerende aandoening omdat voor hen stoppen met roken nog belangrijker is dan voor (vooralsnog) gezonde rokers. 
We waren niet alleen erin geïnteresseerd of de confronterende counseling effectief was maar ook hoe de interventie zou werken. Toen de interventie ontwikkeld werd was de veronderstelling dat confronterende counseling bij rokers - door labelling met een potentieel levensbedreigende ziekte - de risicoperceptie op een aan roken gerelateerde ziekte en de bezorgdheid over de gezondheid zou vergroten alsmede het gebruik van smoesjes om het roken goed te praten zou verminderen. Deze veranderingen in cognities zouden het effect van de interventie op stoppen-metroken mogelijk kunnen verklaren. Om deze veranderingsmechanismen te analyseren werd een mediatieanalyse uitgevoerd van het effect van confronterende counseling op de korte termijn abstinentie van roken bij de 228 rokers uit de experimentele groep en controlegroep 1 van de COSMO studie (hoofdstuk 8). De cotinine gevalideerde stop-roken percentages 5 weken na de geplande stopdatum waren $43,1 \%$ in de experimentele groep vergeleken met $31,3 \%$ in controlegroep 1 ( $O R=1,67$, $95 \% \mathrm{BI}=0,97-2,87)$. Het effect van confronterende counseling op abstinentie werd onafhankelijk gemedieerd door een toegenomen verwachting een ernstige aan roken gerelateerde ziekte te ontwikkelen $(\mathrm{OR}=1,76,95 \% \mathrm{BI}=1,03-3,00)$, een afname van het gebruik van smoesjes om het roken goed te praten $(O R=0,42,95 \% B I=0,21$ $0,84)$, en een vergroting van de eigen effectiviteit $(O R=1,38,95 \% B I=1,11-1,73)$.

Los van de effectiviteit van de aanpak is het de moeite waard om na te gaan of vroegtijdig opsporen van COPD en labelling ethisch gerechtvaardigd is. Labelling met ziekte - het confronteren van mensen met een voorheen niet bekende of gediagnosticeerde gezondheidsstatus - is sinds lange tijd een punt van discussie in de geneeskunde. In de meeste gevallen richtte zich de discussie op de verwachte negatieve effecten van labelling, maar labelling kan ook positieve effecten hebben (labelling zou roker met COPD bijvoorbeeld kunnen motiveren om met roken te stoppen). Semi-gestructureerde ethische exit interviews over dit onderwerp werden bij 205 deelnemers van de COSMO studie afgenomen om hun meningen in kaart te brengen over de effectiviteit van spirometrie voor stoppen-met-roken, de rechtvaardigheid van vroegtijdig opsporen van luchtwegobstructie en de impact van confrontatie met COPD (hoofdstuk 9). Onze resultaten lieten zien dat labelling met ziekte waarschijnlijk een minder belangrijk punt is in de discussie over de voor- en nadelen van vroegtijdig opsporen van COPD; de meerderheid van de deelnemers (86\%) was het ermee eens dat het gerechtvaardigd is om de longfunctie te meten bij zware rokers. Deze deelnemers beargumenteerden dat longfunctiemeting het bewustzijn vergroot over de schadelijke effecten van roken, dat het helpt om ziekte te voorkomen of dat het de motivatie om te stoppen met roken kan vergroten. De meeste deelnemers die het hiermee niet eens waren gaven als reden aan dat het meten van longfunctie bij rokers in de dagelijkse praktijk in strijd is met de eigen keuzevrijheid.

Afgezien van de COSMO studie deden we observationeel onderzoek naar het stoproken beleid van Nederlandse gezondheidszorgwerkers. Volgens nationale en inter- 
nationale richtlijnen over de behandeling van tabaksverslaving kunnen hulpverleners in de gezondheidszorg, zoals huisartsen, cardiologen, longartsen of longverpleegkundigen een belangrijke rol spelen in het terugdringen van het roken. Het is echter niet duidelijk wat ze gewoonlijk doen aan stop-roken behandeling en wat hun attitudes en ervaren effectiviteit zijn. Om daarover duidelijkheid te krijgen voerden we nationale vragenlijstonderzoeken uit onder 834 huisartsen, 300 cardiologen, 258 longartsen (deze onderzoeken zijn gecombineerd in hoofdstuk 2) en 254 longverpleegkundigen (hoofdstuk 3). De resultaten toonden aan dat de prevalentie van roken onder Nederlandse artsen en longverpleegkundigen sterk gedaald is in de afgelopen jaren en relatief laag is (4-8\%) ten opzichte van het landelijk gemiddelde (29\%). Van de farmacologische hulpmiddelen voor stoppen-met-roken bevolen artsen voornamelijk bupropion aan, gevolgd door nicotine pleisters en nicotine kauwgom. Verder werd het gebruik van deze drie middelen vaker aanbevolen door longartsen dan door huisartsen en cardiologen. De longverpleegkundigen bleken het Nederlandse protocol voor de behandeling van nicotine en tabaksverslaving (de zogenaamde "L-MIS") goed op te volgen; zeven van de tien gedragsmatige technieken voor stoppen-metroken uit dat protocol werden door meer dan $94 \%$ van de respondenten gebruikt. Een waargenomen gebrek aan motivatie van de patiënt was de meest belangrijke barrière voor longverpleegkundigen met betrekking tot de behandeling van roken. Gedragsmatige behandelingen voor stoppen-met-roken zijn mogelijk beter haalbaar voor longverpleegkundigen dan voor artsen die hiervoor vaak te weinig tijd hebben.

Zoals reeds eerder aangegeven rookt een aanzienlijk deel van de bevolking ondanks de bekende schadelijke gevolgen van roken voor de gezondheid. Daarnaast bestaat er een grote sociaaleconomische gradiënt ten aanzien van roken in ontwikkelde landen; de hoogste prevalentie van roken wordt doorgaans gevonden onder mensen die half- of ongeschoolde handarbeid verrichten (44\% in Nederland in 2007) en de laagste prevalentie onder hooggeschoolde werknemers (27\%). Er wordt aangenomen dat deze gradiënt verantwoordelijk is voor de helft van alle socio-economische verschillen in mortaliteit in mannen met een leeftijd tussen de 35-69 jaar in ontwikkelde landen. Het is een belangrijk toekomstig doel om sociale gezondheidsverschillen te verminderen die veroorzaakt worden door roken. Een afname van de verschillen in de prevalentie van roken vereist beter inzicht in de onderliggende factoren. We gebruikten data van 6.950 respondenten van een Brits cross-sectioneel onderzoek om de variatie tussen sociale klassen te onderzoeken ten aanzien van: de proportie rokers dat een stoppoging onderneemt, het gebruik van farmacologische of gedragsmatige ondersteuning voor stoppen-met-roken en de proportie succesvolle stoppers (hoofdstuk 10). In totaal hadden 2.983 respondenten een poging ondernomen om te stoppen met roken gedurende het voorafgaande jaar, en 469 waren gestopt ten tijde van het onderzoek. De resultaten toonden geen verschillen aan tussen sociale klassen wat betreft het percentage rokers dat een stoppoging had ondernomen $(42,7 \%$ in de hoogste sociale klasse $A B$ tot $41.2 \%$ in de laagste sociale 
klasse E), het gebruik van farmacologische hulpmiddelen voor stoppen-met-roken (46,7\% van de rokers die een stoppoging hadden ondernomen in $A B$ tot $50,9 \%$ in $E$ ) of het gebruik van de Stoppen-met-roken diensten van de nationale gezondheidszorg $\mathrm{NHS}$ (7,0\% van de rokers die een stoppoging hadden ondernomen in $A B$ tot $4,8 \%$ in E). Er was echter een groot verschil in succespercentages: 20,4\% van de rokers die een stoppoging hadden ondernomen in sociale klasse $A B$ was nog steeds niet-roker ten tijde van het onderzoek ten opzichte van $11,4 \%$ in sociale klasse E). 


\section{Acknowledgements}


Conducting the "Confronting Smokers with COPD" (COSMO) study and finishing this $\mathrm{PhD}$ thesis would have been impossible without the help of many people.

First of all I would like to thank my supervisors Onno van Schayck, Geertjan Wesseling and Marcus Huibers. Onno, thank you for the good and intensive collaboration; you permanently motivated and supported me throughout the years, which was most important to me. I have learnt a lot from you, and laughing with you made work much more fun. Geertjan, you have opened the doors to the clinical practice network in the Maastricht region, and your feedback as a physician has given meaning to my theoretical work. Thank you for your inspiration and your support (and for showing me how to play golf). Marcus, thank you for joining the COSMO project and being there for me in good and in bad times. Your analytic, epidemiologic and writing skills have improved my work cosiderably.

I want to thank "my ladies" from the COSMO research team: Kitty van der Meer, Arja van de Voorde and Ellen de Goeij. Looking back, it is impressing how much work all of you have done for this project; calling, planning, mailing, screening and measuring many hundreds of people. Thank you very much for making this project a success!

An essential part of the COSMO study was the counselling of smokers by the respiratory nurses Marleen Vaassen, Hilde Bastiaens, Karin Frambach, Tim Sales and Rianne Römers. Combining your duties at the university hospital with your work for the COSMO project wasn't always easy. Thank you for your commitment and the very good job you have done.

Several other people helped me a lot with conducting the COSMO study and with the work on my PhD thesis. Erie van den Heuvel, thank you for managing the project and helping me almost immediately with every problem - you are indispensable. Willem de Goeij, what would I have done without the database you have built for COSMO? Thank you very much for the dedication with which you worked on this project and all your collateral advice. Lou Maas, thank you for analyzing the urine cotinine of the COSMO participants. Arnold Kester, thank you for your statistical advice on the randomisation procedure of COSMO. Mischa Aussems, thank you for training people in performing spirometry and for all your work on validating hundreds of spirometric test results. Hendrikus Boersma, thank you for your help with repacking and distributing the study medication for COSMO. The ladies at the office of the Department of Respiratory Medicine of Maastricht University Medical Centre, in particular Nicole Smeets-Genders and Manon Pesik-Hijnens, thank you for the correspondence with the GPs who were involved in the COSMO study. Tanja de Bruijn, thank you for planning the many meetings of the COSMO project team and especially for helping me organising the symposium and promotion. Marga van der Aa and Jim Tatipata, thank you for your IT assistance. The people from MEMIC, especially 
Annemie Mordant, Nelleke de Meij, Paula Rinkens, and Anita Legtenberg, thank you for entering the extensive data-entry for the COSMO project. Ger Driessen, thank you for helping me with cleaning the data sets from COSMO; it is amazing to see how clever you handle data. Berna Schouten, thank you for helping out with screening and planning of COSMO participants. Kristin Kotz and Ursula Hovet, thank you for proofreading my PhD thesis (how confronting this must have been to you, Ursula, as you are a smoker yourself). Patty Nelemans, thank you for your collaboration on our external validation study and for your very nice speech during my graduation. Rein Vos, thank you for investing so much time in working on the qualitative data from the COSMO trial. Edwin Wagena, thank you for giving me the opportunity to doing research while I was still a student and for putting me forward as a PhD-student on the COSMO project. Bas Verhage, thank you for giving the final touch to my PhD thesis by designing the cover - a task with a high responsibility as this page is probably the most prominent one of the whole thesis. I admire your creativity and your skills as a designer.

I would also like to thank the members of the reading committee of my PhD thesis and the corona for their time and interest in my work: IJmert Kant, Dirkje Postma, Hein de Vries, Robert West, and in particular André Knottnerus for chairing the reading committee. Richard Dekhuizen, thank you for joining the corona. And special thanks to Robert West, Jan Degryse and Marc Willemsen for their agreement to come to Maastricht and to speak at the symposium.

The COSMO project was sponsored by the Netherlands Asthma Foundation, Partners in Care Solutions for COPD (PICASSO - an initiative of the School for Public Health and Primary Care (CAPHRI), Boehringer Ingelheim, and Pfizer), and Maastricht University Medical Centre. I would like to thank these funding organizations for their support and Edmee van den Akker, Therese Noorlander and Guus Asijee for the good collaboration.

Special thanks to my two "paranimfen" Mark Spigt and Jochen Cals. I thank you not only for your help during the preparation of the PhD defence and for your support on that day but also for having been important companions during my 4.5-year PhD journey. It's good to have both of you as colleagues and friends.

We spend at least one third of our life at work; how depressing would this life be without nice colleagues? I have enjoyed working at Randwyck, in particular because of Janneke Kaper, Ludovic van Amelsvoort, Wolfgang Viechtbauer, Stijn Soenen, Ellen Leers, Sil Aarts, Sjoerd Hobma, Anja Schaefer, Annie Hendriks, Marlie Leerssen, Hans-Jürgen Bruns, Saskia Duijts, Susanne Hanssen, my other colleagues from the Department of General Practice and the PhD-students from the DEB1 PhD meeting (formerly known as POEH). 
I would also like to thank all the members and guests of the "Journal Club Mesch" (JCM: www.hag.unimaas.nl/jcm) for making this initiative such a success, especially Mark Spigt and Maud Kertzman for being such wonderful hosts. I think all members of the JCM can be proud of this special initiative which broadens our scientific mind and facilitates collaborations across disciplines and departments. I am looking forward to many more interesting scientific debates and entertaining evenings.

It is a great opportunity for a PhD-student to go abroad and work with well-known researchers in the field. In October 2006, I attended a workshop at Dave Sackett's "Trout Research Centre" in Ontario, Canada. Thank you Dave and Barbara for your great hospitality! It was a great pleasure meeting you and working with you. I'd also like to thank the European Centre of Excellence in Clinical Tobacco Research for inviting me to Prague in June 2007. That meeting really left a mark on me - in several ways. During the months of September and October 2007, I worked in London at the Cancer Research UK Health Behaviour Unit (HBU) of University College London. Thanks to the European Respiratory Society for financing this research fellowship. Working at the HBU and living in London was a very special experience to me. I would like to thank the HBU for hosting me and in particular Jenny Fidler, Eleni Vangeli, and Ellen van Jaarsveld for having such a good time. I am very grateful that this fellowship has resulted in an ongoing collaboration.

Should I also thank "die Mannschaft" for usually performing better than the Dutch national football team at international championships? Well, maybe because it prevents me of being bullied at work. And if my linear regression analysis is correct I can expect a bright future: FIFA championship 2006: $3^{\text {rd }}$ place, UEFA championship 2008: $2^{\text {nd }}$ place, FIFA championship 2010:

Last but not least, I would very much like to thank my family and friends. Johannes and Kristin, you are wonderful parents. What I have achieved in my life so far would hardly have been possible without your unlimited support. I may not often have articulated this explicitly, but your support is very important to me.

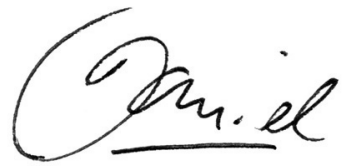

Maasticht, October 2008

PS. So many people have been involved in this $\mathrm{PhD}$ project; I might have forgotten to acknowledge somebody here of which I am terribly sorry. If it is you, please put your name down here:

I would also like to thank: 


\section{About the author}


Daniel Kotz was born in Vreden, Germany, on 16 April 1976. He passed secondary school in Vreden in 1995. Convinced by his mother that he shoud challenge his hands as well as his brains, he moved to Nijmegen, the Netherlands, in 1996 to study physiotherapy at the University of Professional Education Arnhem and Nijmegen (HAN) where he received his diploma in 2000. He then worked for one year as coordinator and physiotherapist at Seneca, the centre for sport and health of the HAN. Being actually more interested in theory than in clinical practice, he moved to Maastricht, the Netherlands, in 2001 to study health science at Maastricht University and received his master's degree in 2003 (major subject work and

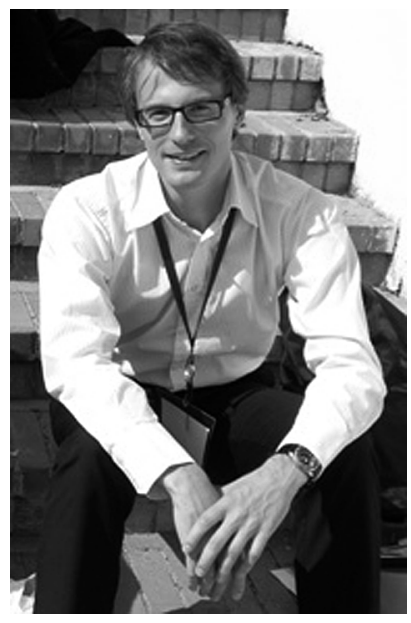
health). During this period, he became interested in science and started his career in the field of tobacco research as a junior researcher at the School for Public Health and Primary Care (CAPHRI) of Maastricht University Medical Centre (MUMC). In 2004, he began his work as a health scientist and PhD student at CAPHRI. His primary research project was the design and conduction of a randomized controlled trial on the efficacy of smoking cessation treatments in smokers with previously undetected airflow limitation (the "COSMO" study). He also worked as a $\mathrm{PhD}$ representative of CAPHRI from 2004 to 2007, as well as a delegate in the general and social committee (now known as the PhD Academy) of PROVUM, the organization for PhD students at Maastricht University. Concurrently, he studied epidemiology at the MUMC and received his second master's degree in 2006 (with distinction). In the same year, he went to Canada to work with David Sackett, a pioneer in evidencebased medicine. When he came back, he co-founded the Journal Club Mesch, which is a bi-monthly discussion group of junior and senior researchers from MUMC, working in various fields of human health research (www.hag.unimaas.nl/jcm). In 2007 he participated in the European Centre of Excellence in Clinical Tobacco Research and worked with a grant from the European Respiratory Society as a research fellow at the Cancer Research UK Health Behaviour Unit of University College London, together with Robert West, which resulted in a continuous collaboration. His PhD thesis was approved in July 2008. He acquired a Kootstra Talent Fellowship from MUMC and started his work as a post-doc and epidemiologist at the MUMC in August 2008. In September 2008 he became a fellow of the Brisbane Initiative, which is an international primary care research leadership programme. His current research interests include behavioural and pharmacological treatment of nicotine and tobacco addiction, epidemiology of chronic obstructive pulmonary disease and research methodology and statistics in general. In his private life, he likes doing sports (especially mountain biking in the Sauerland), spending quality time with his friends and 
being the godfather of Joos-Henrik Hörnemann and Anni Rölver. He is also singer, songwriter and guitarist of the band "Andromedan Funk Machine" and is considering a second career as a world famous funk-rock musician in case his scientific career does not work out as he hopes.

More information: www.daniel-kotz.de 



\section{List of publications}


Cals, J.W.L \& Kotz, D. Researcher identification: improving the search for the right needle in the haystack. The Lancet 2008; 371: 2152-2153

Kotz, D., Nelemans, P., Van Schayck, C.P. \& Wesseling, G.J. External validation of a COPD diagnostic questionnaire in smokers. European Respiratory Journal 2008; 31: 298-303

Kotz, D., van Litsenburg, W., van Duurling, R., van Schayck, C.P. \& Wesseling, G.J. Smoking cessation treatment by Dutch respiratory nurses. Reported practice, attitudes and perceived effectiveness. Patient Educ Counsel 2008; 70: 40-49

Kotz, D., Huibers, M.J.H., Vos, R., Van Schayck, C.P. \& Wesseling, G.J. Principles of confrontational counseling in smokers with chronic obstructive pulmonary disease (COPD). Med Hypothesis 2008; 70: 384-386

Kotz, D., van de Kant, K, Jöbsis, Q, et al. Effects of tobacco exposure on lung health and pulmonary biomarkers in young, healthy smokers aged $12-25$ years: a systematic review. Expert Rev Resp Med 2007; 1:403-418

Kotz, D., Wesseling, G.J., Huibers, MJH, et al. Efficacy of confrontational counselling for smoking cessation in smokers with previously undiagnosed mild to moderate airflow limitation: study protocol of a randomized controlled trial. BMC Public Health 2007; 7:332

Kotz, D., Van Schayck, C.P., Huibers, M.J.H. \& Wesseling, G.J. Assessing the efficacy of spirometry for smoking cessation. Thorax 2007; 62 (8):742

Spigt, M.G. \& Kotz, D. Comment on: "A simple and valid tool distinguished efficacy from effectiveness studies". Journal of Clinical Epidemiology 2007; 60 (7): 753-755

Kotz, D., Wagena, E.J., Wesseling, G.J. Smoking cessation practices of Dutch general practitioners, cardiologists, and lung physicians. Respiratory Medicine 2007; $101(3): 568-573$

Kotz, D. \& Kaper, J. Stoppen met roken in de huisartspraktijk. [Smoking cessation in general practice.] Huisarts Nu 2006, 35(3) 
Kotz, D. \& Wagena, E.J. Roken en stoppen met roken in de eerste en tweede lijn van de gezondheidszorg. Attitudes, gedrag en eigen effectiviteit van huisartsen, longartsen en cardiologen. [Smoking and smoking cessation in primary and secondary health care. Attitudes, behaviour an self-efficacy of general practitioners, lung physicians and cardiologists.] Maastricht: Maastricht University

\section{Manuscripts submitted/accepted for publication}

Kotz, D., Wesseling, G., Huibers, M.J.H. \& Van Schayck, C.P. Confronting smokers with previously undetected COPD does not increase prolonged smoking cessation rates: a randomized controlled trial

Kotz, D., Huibers, M.J.H., West, R.J., Wesseling, G. \& Van Schayck, C.P. What mediates the effect of confrontational counselling on smoking cessation in smokers with COPD?

Kotz, D., Vos, R. \& Huibers, M.J.H. Ethical analysis of the justifiability of labelling with COPD for smoking cessation

Kotz, D. \& West, R. Explaining the social gradient in smoking cessation: it's not in the trying, but in the succeeding. (accepted for publication in Tobacco Control)

Yanbaeva, D.G., Dentener, M.A., Spruit, M., Houwing-Duistermaat, J., Limapassos, V, Kotz, D. \& Wouters, E.F.M. Genetic variation in genes coding for inflammatoryrelated proteins (CRP, FGB and IL6) and COPD: tagging SNP analysis in a casecontrol study 
